KRL MAP-247

\title{
EFFECTIVE FIELD THEORY OF NUCLEAR FORCES
}

\author{
U. van Kolck \\ Kellogg Radiation Laboratory, 106-38 \\ California Institute of Technology \\ Pasadena, CA 91125, USA \\ vankolck@krl.caltech.edu
}

\begin{abstract}
The application of the effective field theory (EFT) method to nuclear systems is reviewed. The roles of degrees of freedom, QCD symmetries, power counting, renormalization, and potentials are discussed. EFTs are constructed for the various energy regimes of relevance in nuclear physics, and are used in systematic expansions to derive nuclear forces in terms of a number of parameters that embody information about QCD dynamics. Two-, three-, and many-nucleon systems, including external probes, are considered.
\end{abstract}

${ }^{*}$ Commissioned for Prog. Part. Nucl. Phys. 


\section{Contents}

\begin{tabular}{llr}
\hline Introduction & 1
\end{tabular}

II EFT

A What is effective? . . . . . . . . . . . . . . . . . . . 5

B Not too much freedom . . . . . . . . . . . . . . . . . . . . . . . 8

C Symmetries . . . . . . . . . . . . . . . . . . . . . 9

D The power of counting . . . . . . . . . . . . . . . . . . 15

E $\quad$ Propaganda . . . . . . . . . . . . . . . . . . . . . . . . . 18

\begin{tabular}{llr}
\hline III & One- or no-nucleon processes & 18
\end{tabular}

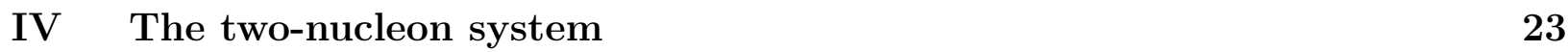

A Very low energies . . . . . . . . . . . . . . . . . . . . . . . . . . . . . . . . . 3

B $\quad$ Low energies . . . . . . . . . . . . . . . . . . . . . . . . . . . . . . . 39

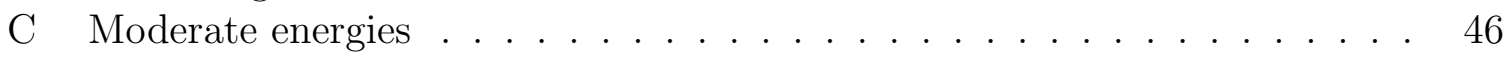

\begin{tabular}{|l|l|l|l|l}
\hline Few-nucleon systems & 54
\end{tabular}

A Very low and low energies . . . . . . . . . . . . . . . . . . . . . . . . . . . . . 55

B Moderate energies . . . . . . . . . . . . . . . . . . . 63

\begin{tabular}{lll}
\hline VI & Probes of few-nucleon systems & 66
\end{tabular}

A Very low and low energies . . . . . . . . . . . . . . . . . . . . 66

B $\quad$ Moderate energies . . . . . . . . . . . . . . . . . . . . . . . 68

\begin{tabular}{|lc}
\hline VII Many-nucleon systems & 73 \\
\hline
\end{tabular}

\begin{tabular}{|lc}
\hline VIII Outlook & 74 \\
\hline
\end{tabular} 


\section{INTRODUCTION}

There is little doubt that QCD is the correct theory of the strong forces that bind quarks into baryons and mesons and yet, despite many efforts, it has not been possible to derive the interaction among these composite states from first principles. At the root of the problem is our inability to solve the dynamics of QCD at hadronic scales because of a large coupling constant. Much of the description of hadronic properties and interactions has been limited to phenomenological models whose connections with QCD are unclear.

The strong-coupled dynamics among baryons is particularly rich: it gives rises to bound states, of which ordinary nuclei are particular interesting. A superficial look at hadronic and nuclear tables conveys complexity, but a closer look reveals some regularities that can be translated into different mass scales. At least two scales influence the bulk of the dynamics in a nucleus. The masses of the main constituents (nucleons) and their excitations (chiefly the delta isobar), the masses of most mesons that can be exchanged among them (the rho, omega, etc.), and the chiral symmetry breaking scale; they all cluster near what we can call the characteristic QCD scale, $M_{Q C D} \sim 1 \mathrm{GeV}$. On the other hand, the reciprocal of the sizes of light nuclei, the Fermi momentum of equilibrium nuclear matter, the pion mass and decay constant, and the delta-nucleon mass difference take values closer to a lighter scale $M_{n u c} \sim 100 \mathrm{MeV}$. From these two scales, a third, $M_{n u c}^{2} / M_{Q C D} \sim 10 \mathrm{MeV}$, manifests itself in the order of magnitude of nuclear binding energies.

In nuclear physics there is thus a natural separation of scales, and one can hope to use it to simplify the theoretical analysis. Such an approach has proved useful in other areas of physics, and is at the heart of the use of renormalization group techniques. The method of effective field theory (EFT) evolved from these techniques, and it was designed to incorporate and benefit from the existence of separate scales, even when couplings are not small compared to 1. It seems to constitute an ideal tool for low-energy nuclear physics, a tool which has similarities but also significant differences compared to the more traditional phenomenological approach. The method is firmly based on the known (approximate) symmetries of QCD, but parametrizes our ignorance of dynamical details in a number of undetermined constants. This article is a review of the program of research that attempts to describe nuclei and processes involving them using the method of EFT.

The scales described above are consistent with a picture of nuclei as composed of nonrelativistic nucleons of typical momenta $Q \sim M_{n u c}$ and energies $Q^{2} / M_{Q C D}$. Now, slow particles make for poor microscopes. A nucleon in the nucleus most of the time cannot resolve features with a spatial extent $\lesssim 1 / Q$. In particular, particles that live for short times and distances can in first approximation be treated as points. Only those particles that propagate over distances longer than $\sim 1 / Q$ need to be retained in the theory as explicit degrees of freedom. They will supply the correct low-energy analytic structure (poles and cuts) of physical amplitudes. This is a significant simplification, as it means that a large number of states (massive conventional hadrons or exotic quark states) can be accounted for just through their contributions to local interactions. Further simplification follows from the fact that we have been able to identify a number of symmetries of QCD, as well as the pattern of their breaking. Low-energy $S$-matrix elements have to exhibit the same symmetry pattern, which markedly constrains the possible interactions between the relevant degrees of freedom. This still leaves us with infinitely many local interactions, as arbitrary numbers 
of derivatives appear to account for corrections to the point approximation. Worse still, parameters characterizing the interaction strengths need not be small. The crux of the EFT method is to find a way to organize this infinite set of interactions using an expansion in the small parameter $Q$.

The method can be illustrated with a very familiar example. Let us suppose we want to study a system whose overall size is $R_{l}$ but that contains smaller structures of size $R_{s} \ll R_{l}$. We can do so by shining light of wavelength $\lambda$ onto the system, but for my purposes here it does not really matter if this "light" comes from a laser source or from electron scattering or whatever; in fact I will consider just a static field, the only important thing being that there is a scale $\lambda$ associated with its (spatial) variation. We can learn about the system because its charge density $\rho(\vec{r})$ affects the interaction energy between the system and light.

Let us consider first the case when $\lambda \gg R_{l}$. In classical electrodynamics we argue that the interaction energy can under these circumstances be written as a multipole expansion. In first approximation, we need only to know the overall charge $q$ of the system and the electromagnetic potential at its center. In second approximation we need also the system's dipole moment and the gradient of the potential, and so on. Schematically,

$$
\int d^{3} r \rho(\vec{r}) \phi(\vec{r})=q \sum_{n=0}^{\infty} c_{n}\left(\frac{R_{l}}{\lambda}\right)^{n} .
$$

This expansion is justified by dimensional analysis: except for very pathological charge distributions, the interaction energy is analytic in $1 / \lambda$ and successive terms must come in powers of $R_{l} / \lambda$ times dimensionless factors $c_{n}$ of $O(1)$. The latter encode all we need to know about the system; they can be calculated from $\rho(\vec{r})$, but if $\rho(\vec{r})$ is not known, we do not have to weigh on every single dimple of the charge distribution. To a given accuracy, we can truncate the multipole expansion and parametrize the electromagnetic properties of the system by a finite number of parameters instead of a continuous $\rho(\vec{r})$. The role of an exact (approximate) symmetry is that it forbids (suppresses) certain terms in the expansion. Now, all this can be translated to QED. All we need to do is to write the most general Lagrangian allowed by gauge invariance that involves fields for the system (and perhaps its excited states) and the photon. This Lagrangian will contain not only minimal couplings governed by charge but also operators with more derivatives, such as a Pauli term. The rules of field theory then can be used to derive the electromagnetic form factors of the target — or more generally any scattering amplitude - with contributions ordered by $R_{l} / \lambda$. There is a difference, however. Quantum fluctuations from loops can probe small distances. The procedure of renormalization is designed to ensure that the physics of these small distances contribute to the physics of large distances only in an average sense, and that the multipole expansion remain valid. Left behind are some calculable cuts. Because (point) charge interactions are renormalizable, the non-analytic terms are suppressed only by powers of the fine-structure constant, $\alpha$. Loops involving higher-derivative operators are further suppressed by powers of $R_{l} / \lambda$.

Consider now an increase in resolution: $\lambda \sim R_{l}$. Again, because $\lambda \gg R_{s}$ the contributions of the small structures can be treated in an expansion in $R_{s} / \lambda$ as in Eq. (11), except that the multipole parameters now refer to the inner charge distribution of charge $q_{s}$. Contributions from the outer cloud, on the other hand, can only be accounted for by the full knowledge of the outer distribution $\rho_{l}(\vec{r})$. The interaction energy is now 


$$
\int d^{3} r \rho(\vec{r}) \phi(\vec{r})=q_{s} \sum_{n=0}^{\infty} c_{n}^{(s)}\left(\frac{R_{s}}{\lambda}\right)^{n}+\int d^{3} r \rho_{l}(\vec{r}) \phi(\vec{r}) .
$$

In the quantum context these outer contributions that are non-analytic in $1 / \lambda$ come from loops generated by the light particles flying within the target at the time light strikes. The magnitudes of loop diagrams will depend crucially on the interactions among the structures that make up the system. In general, they are not small compared to tree-level graphs.

We can translate this simple example to a typical nucleus, where $M_{Q C D} \sim 1 / R_{s}$, $M_{n u c} \sim 1 / R_{l}$, and $Q \sim 1 / \lambda$. It turns out that in this case we can justify not only the "multipole" or derivative expansion, but we can control the loop expansion as well.

The first (and simplest) situation, $Q \lesssim M_{n u c}$, corresponds to nucleons of momenta sufficiently small that all interactions among nucleons can be described as contact interactions. One might be tempted to dismiss this as an uninteresting case, but it is not so, as some information on the two-nucleon system reveals. At low momenta the scattering amplitude in both isospin channels $(I=0$ and $I=1)$ can be very well-described by the effective range expansion. One finds that, as expected on the basis of dimensional analysis, most of the $S$ wave effective range parameters scale with $M_{n u c}^{-1}$. However, the scattering lengths $a_{2}$ are large and reflect the existence of a new, smaller mass scale which I denote by $\aleph \sim 1 / a_{2}$. In the ${ }^{3} S_{1}$ channel, $\aleph^{\left({ }^{3} S_{1}\right)} \sim 40 \mathrm{MeV}$, somewhat smaller than $M_{n u c}$. In the ${ }^{1} S_{0}$ channel, $\aleph^{\left({ }^{1} S_{0}\right)} \sim 8 \mathrm{MeV}$ $\ll M_{n u c}$. For simplicity, I take an average $\aleph \sim 30 \mathrm{MeV}$. The existence of a new scale requires some amount of fine-tuning. whatever its origins, the consequence is that these channels have anomalously shallow bound states with binding energies $\sim \aleph^{2} / M_{Q C D} \ll M_{n u c}^{2} / M_{Q C D}$ In the singlet channel this bound state is virtual, but in the triplet it is real - the deuteron.

The idea of a low-energy expansion is in fact as old as nuclear physics: already in the 30's Bethe and Peierls [1] reasoned that, because the deuteron has a large size $\lambda$ compared to the range $R_{l}$ of nuclear forces, up to an error $O\left(R_{l} / \lambda\right)$ the sole effect of the potential is to provide an energy-independent boundary condition at $r \sim R_{l}$ to an otherwise free Schrödinger equation. This can be recast in terms of an EFT of non-relativistic nucleons -non-relativistic because $Q$ is small compared to the nucleon mass. As we will see in detail below, interactions contain $\aleph$ besides $M_{n u c}$ and the appearance of $Q^{2} / M_{Q C D}$ as the only scale in energy denominators of loop diagrams generates enhancements that require a resummation of a large class of diagrams. After resummation, the EFT includes terms of all orders in $Q / \aleph$ but is perturbative in $Q / M_{n u c}$. Although the EFT in the two-body case is little more than a generalization of the technique used by Bethe and Peierls, it allows an easy extension of these arguments to processes where low-energy photons, electrons and neutrinos probe the deuteron. It also allows the study of nucleon-deuteron scattering at low-energies, and perhaps of the triton and other nuclei as well. I will refer to momenta $Q \lesssim M_{n u c}$ as "very low".

As we increase momenta and $Q \sim M_{n u c}$, pions can no longer be considered heavy, as $Q$ is comparable to their mass. The EFT has to be augmented to include them as explicit degrees of freedom. (Because the delta-nucleon mass difference is of the same order of magnitude, explicit inclusion of the delta might also be called for.) Explicit inclusion of the pion dynamics gives rise to non-analytic terms analogous to those in our simple electrodynamics example. All short-range dynamics from massive states can be treated in an expansion in $Q / M_{Q C D}$, and is parametrized in analogy to the $c_{n}^{(s)}$ in Eq. (2). What is 
crucial for the success of the approach is that one can show that - in a sense to be made more precise below - pion loops can also be ordered in terms of an expansion in $Q / M_{Q C D}$. In terms of Eq. (2), this would mean that, apart from logarithms and dimensionless factors of $O(1)$, contributions from the outer cloud would come with a small factor $\left(R_{l} / 4 \pi \lambda\right)^{2}$ for each loop, namely

$$
\int d^{3} r \rho_{l}(\vec{r}) \phi(\vec{r})=q_{l} \sum_{L=0}^{\infty} \gamma_{L}\left(\frac{R_{l}}{4 \pi \lambda}\right)^{2 L}
$$

where $\gamma_{L}$ are calculable functions of the Lagrangian parameters. It is chiral symmetry that effects this magic by requiring that pion interactions be proportional to $Q$, and therefore weak at low energies.

The EFT appropriate to these momenta has been extensively applied to systems containing one or no nucleon, and in that context it is called Chiral Perturbation Theory $(\chi \mathrm{PT})$. The application of $\chi \mathrm{PT}$ to nuclear systems is complicated by the resummation needed to accommodate the infrared enhancement. What class of diagrams is to be re-summed? The answer to this question depends on the relative magnitude of pion and shorter-range effects. It is reasonable that at sufficiently small $Q$, pions can be treated perturbatively, while as $Q$ reaches some scale $M_{N N}$ pion effects become non-perturbative. If $M_{N N}$ is as low as $M_{n u c}$, then the theory with perturbative pions is of little use, since then the pion can be simply replaced by a point. If $M_{N N}$ is as high as $M_{Q C D}$, then non-perturbative pions are not necessary as long as $Q<M_{Q C D}$ [. I will refer to momenta $Q \lesssim M_{N N}$ as "low" and to momenta $M_{N N} \lesssim Q \lesssim M_{Q C D}$ as "moderate". (What the scale $M_{N N}$ is is a matter of current debate.)

What is unique about the application of EFT to nuclear physics is this fascinating interplay between perturbative and non-perturbative phenomena. EFTs already have a long history, as its first example likely is the work of Euler and Heisenberg in QED [2]. However, its modern concept evolved only during the late 60's and the 70's, as a confluence of several ideas. First, in $S$-matrix days, it was noticed [3] that a chiral Lagrangian used at tree level was a field theoretical realization of current algebra. Second, the spectacular experimental successes of the Standard Model together with its only partially unified structure suggested that it be viewed as the low-energy remainder of a more fundamental theory [ 4 . Third, a new view of renormalization grew out of renormalization group ideas [5]. By the end of the 70's it was clearly realized that the concept of effective Lagrangians provides the rationale behind the use of field theory in particle physics phenomenology [6,7]. It was in the 80's that

${ }^{\dagger}$ Currently, the only way to deal with processes with momenta $Q \sim M_{Q C D}$ is by phenomenological models. Those (few) models which include QCD symmetries correctly can be roughly thought of as EFTs in which an infinite number of operators is assumed to be correlated and their parameters functions of the few model parameters. Both model-independence and a systematic expansion are lost. Truly extending the EFT approach to such "high" momenta presents formidable obstacles. First, the number of relevant degrees of freedom increases tremendously. Second, one would need to find a new small expansion parameter. I do not attempt to review efforts in this direction here, as they have had no clear successes in nuclear physics yet. 
a detailed, systematic analysis of low-energy hadronic physics based on EFT began [8,9], but even then, no non-perturbative effects were dealt with.

Here I will consider mainly the description of nuclear systems per se for $Q<M_{Q C D}$, a program that was initiated in Refs. 10 18. This early work has shown that many of the observed but otherwise mysterious features of nuclear physics can be naturally understood in this framework 15,19]. More recently, several aspects of this program have received vigorous attention - for a sample, see Ref. [20] - and a reasonable body of successes has been amassed. A review seems timely. However, as many review writers before, I am compelled to apologize to those who feel that their work is underrepresented here. Spacetime constraints - the editor's patience notwithstanding - have biased me towards the ideas that I understand better, usually (but not always) my own. For a different perspective on various subsets of the topics I cover here, the reader is invited to consult Ref. 21.

After an introduction to the general ideas of the EFT method in Sect. [I], I briefly present some $\chi$ PT highlights in Sect. III. Sect. $\square$ on the two-body system is perhaps the central one. I consider first the very-low-momentum EFT as its simplicity makes it worth studying; then I delve into pions at low and moderate momentum. Power counting is the theme that has been played but whose scope is not yet completely understood. This sets the stage for the application to few-body systems in Sect. $\nabla$, where remarkable universality emerges. The question we explore is what are the new ingredients brought in by new bodies. Several successful applications to processes involving external probes are mentioned in Sect. V1, and the first, exploratory studies of the many-body system are in Sect. VII]. The balance is in Sect. VIII.

\section{EFT}

Effective field theories exploit the existence of scales in a system. A very brief introduction to the main EFT ideas is presented Subsect. II A. In Subsect. IIB we identify the relevant degrees of freedom whose propagation should be accounted for explicitly in order to supply the correct low-energy analytic structure of physical amplitudes. The symmetries which constrain the low-energy $S$-matrix elements are discussed in Subsect. IIQ. The $S$ matrix can then be generated by the most general Lagrangian involving the relevant degrees of freedom and symmetries. In Subsect. IID one invokes the concept of "natural" size for effective dimensionful parameters in terms of the scale $M$ of the underlying dynamics, in order to establish an ordering of all possible contributions to processes at momenta of order $Q \ll M$ in powers of the small parameter $Q / M$. Some features of the method are stressed in Subsect. [1]E.

\section{A. What is effective?}

What is an effective theory? In order to have a rough idea - a fuller answer can be found in several reviews 222 26] - consider a field theory (which I call the "underlying" theory) given by some Lagrangian $\mathcal{L}_{\text {und }}$ written in terms of some ("elementary") fields $\Psi$; 
and suppose that this theory adequately describes experiments carried out over a certain range of energy $\mathrm{H}$. This means that $S$-matrix elements can be obtained from the path integral

$$
Z=\int \mathcal{D} \Psi e^{i \int \mathcal{L}_{\text {und }}(\Psi)}
$$

We are interested in how such a theory looks like at scales smaller than some scale $\Lambda$ within its range of validity. Excitations with momenta larger than $\Lambda$ cannot be produced directly, so we can profit from splitting the fields $\Psi$ in two components $\Psi_{h}$ ("fast") and $\Psi_{l}$ ("slow"), according to whether their momenta are greater or smaller than $\Lambda$. Integrating over $\Psi_{h}$,

$$
Z=\int \mathcal{D} \Psi_{l} e^{i \int \mathcal{L}_{e f f}\left(\Psi_{l}\right)}
$$

where the effective Lagrangian $\mathcal{L}_{\text {eff }}$ is given, in $D$ spacetime dimensions, by

$$
\int d^{D} x \mathcal{L}_{e f f}\left(\Psi_{l}\right)=-i \ln \int \mathcal{D} \Psi_{h} e^{i \int \mathcal{L}_{u n d}\left(\Psi_{h}, \Psi_{l}\right)}=\int d^{D} x \sum_{i} g_{i}(\Lambda) \mathcal{O}_{i}\left(\Psi_{l}\right)
$$

The operators $\mathcal{O}_{i}$ may be very complicated but they involve only the slowly-varying fields $\Psi_{l}$; the fields $\Psi_{h}$ are said to have been "integrated out". The $\mathcal{O}_{i}$ 's have two important properties. First, they are local in the sense that they involve only fields at the same spacetime point. Yet, they contain arbitrary number of derivatives of such fields. Indeed, according to the uncertainty principle particles with momentum $Q \lesssim \Lambda$ can only probe distances $1 / Q \gtrsim 1 / \Lambda$ and therefore can sense configurations with support smaller than $1 / \Lambda$ only in an average sense. Second, as long as the splitting of fields is done carefully, the $\mathcal{O}_{i}$ 's transform under various groups according to the underlying theory. If the underlying Lagrangian is symmetric under a transformation that is not anomalous, then so is the effective Lagrangian, although the symmetry might be realized non-linearly if it is spontaneously broken. If the symmetry is explicitly broken either at a classical or a quantum level, operators will appear at low energies that incorporate the breaking accordingly. Note, however, that the splitting of fields does require some care. The Lagrangian $\mathcal{L}_{\text {eff }}$ typically contains interactions involving the time derivative of $\Psi_{l}, \dot{\Psi}_{l}$, to powers greater than 1 . In this case there is a non-trivial relation between $\Psi_{l}$ and its conjugate momentum. As a result the interaction Hamiltonian is not simply minus the interaction Lagrangian, but contains additional, Lorentz non-covariant terms. It is no problem to include these interactions, as other non-covariant pieces arise in covariant perturbation theory from contractions involving derivatives, and in time-ordered formalism from its inherent non-covariance. One can show explicitly [27 that the sum of diagrams contributing to any given process is indeed covariant. Alternatively, one can add terms $\Delta \mathcal{L}$ directly to $\mathcal{L}_{\text {eff }}$. For example, for real scalar $\Psi_{l}$, if

$$
\mathcal{L}_{e f f}=\frac{1}{2} \dot{\Psi}_{l}^{T} A\left(\Psi_{l}\right) \dot{\Psi}_{l}+\ldots
$$

then 28

\footnotetext{
$\ddagger$ Most likely the underlying theory is itself an effective theory to an yet more fundamental theory.
} 


$$
\Delta \mathcal{L}=\frac{i}{2} \delta^{D}(0) \operatorname{Tr}\left[\ln \left(A^{-1}\left(\Psi_{l}\right)\right)\right]+\ldots
$$

This Lagrangian is manifestly covariant, but the new non-linear piece generates selfinteractions. These are certainly ill-defined as they stand due to the $\delta^{D}(0)$, but other terms in the unrenormalized Lagrangian are equally ill-defined. The EFT is made well-defined through regularization. $\delta^{D}(0)$ vanishes in dimensional regularization but not when a mass cutoff is used; the ln term is necessary [29] in the renormalization program to get rid of loop contributions that could otherwise spoil the assumed symmetries.

As for the coefficients $g_{i}$, they carry information about details of the dynamics: they are functions of the parameters of the underlying theory, but also depend explicitly on the cutoff $\Lambda$-which lends them the status of "running coupling constants". In diagrams, a change in $\Lambda$ amounts to a reshuffle between contributions from vertices and from the ultraviolet region of loop integration. An obvious requirement is that low-energy observables be insensitive to changes in $\Lambda$, which places constraints on the $\Lambda$ dependence of the $g_{i}$ 's. The equations that govern this dependence are the renormalization group equations.

The notion of an effective Lagrangian is particularly useful when the underlying theory has (at least) one characteristic mass scale $M$. In this case, the effective degrees of freedom for $\Lambda \leq M$ expressed by the fields $\Psi_{l}$ are in general significantly different from the original degrees represented by the fields $\Psi$. It frequently happens that that these low-energy phenomena are "collective excitations" as viewed in terms of $\Psi$ [23]. The reformulation of the theory in terms of $\Psi_{l}$ ends up selecting those effects of the underlying theory that are most important at low energies. The simplest case is the one where $M$ is just the mass of a physical particle. Production and decay of this particle involve large momenta and do not concern the EFT. Effects of virtual exchange (of the particle, or of particle-antiparticle pairs) are of short range and thus only included indirectly in the $g_{i}$ 's [30]. If, furthermore, the particle is not stable in the context of the underlying theory, then it does not (explicitly) appear at all at low energies; we need associate no field $\Psi_{l}$ to it. Another common case is that where $M$ is the scale associated with some (elementary or composed) scalar field acquiring a non-vanishing vacuum expectation value and breaking a (possibly approximate) continuous global internal symmetry group, resulting in the appearance in the spectrum of either a (possibly nearly) massless spin-zero particle [31] - a (pseudo)Goldstone boson- or a massive, longitudinal component of a vector boson [32]. A third case is more complicated: $M$ is a scale where some wild non-perturbative phenomena dominate and force asymptotic states into singlet representations of a local group, an example being confinement in a nonabelian gauge theory with the appropriate matter content. It seems adequate in this case to restrict the effective Lagrangian to fields that are singlets under the gauge group. There are other possibilities (like the existence of a Fermi surface [23,24]), or combinations of various cases, but the important point is, the mass $M$ provides a measure of the strength of the high-energy effects that appear in the coefficients $g_{i}$ 's. This eventually paves the way to a systematic expansion in powers of $Q / M$.

If we know and can solve the underlying theory, then we can calculate the $g_{i}$ 's and obtain the complete form of $\mathcal{L}_{\text {eff }}$ to the accuracy of the approximation involved in the solution. But it frequently happens that we cannot solve the underlying theory (as it is the case of nuclear physics), or that we do not know such theory at all (as it is for the electroweak theory). Are we then justified in using an effective theory? A positive answer is given by a "theorem" 
due to Weinberg [6]: in conciliating quantum mechanics and Lorentz invariance in a way consistent with unitarity, analyticity and cluster decomposition, we are led to quantum field theory; the most general Lagrangian with some assumed symmetries will then produce the most general $S$-matrix incorporating those general principles and symmetries, without any other physical content. This is a statement based on our own currently accessible energy experience: it has only been proved in the particular case of a scalar field with $Z_{2}$ symmetry in Euclidean space [33, but no counter-examples are known.

EFT is therefore modern $S$-matrix theory. All that any theory can do is to relate observables. Field theory based on a Lagrangian is the simplest way to do this given some degrees of freedom and symmetries. The theory is of course regulated by some sort of procedure implementing the cutoff $\Lambda \leq M$ that isolates the neglected degrees of freedom, so diagrams involve only momenta $Q<\Lambda$. Renormalization is carried out as usual, but its aim is not to eliminate infinities: because the EFT is not valid at high energies, we never have to take the $\Lambda \rightarrow \infty$ limit anyway [25]. Rather, the objective is to remove details of the cutoff procedure from physical amplitudes, and to relate Lagrangian parameters and observable quantities 3 . A redefinition of fields in general changes all amplitudes off shell, where they cannot be measured. Only observables, which appear in on-shell amplitudes, are unambiguous in the EFT.

\section{B. Not too much freedom}

The first element of an EFT consists of the identification of relevant low-energy degrees of freedom. Here we will be considering only EFTs for momenta $Q \ll M_{Q C D} \sim 1 \mathrm{GeV}$. This severely restricts the number of degrees of freedom that need to be included explicitly: at such low momenta we cannot probe quark degrees directly, and the number of hadronic states needed is small.

The nucleon mass $m_{N}$ is not light compared to $M_{Q C D}$, but to a very good approximation nucleons cannot decay into lighter states of high momenta. Although we can integrate out nucleon-antinucleon pairs that pop out of the vacuum, a number of nucleons exist in asymptotic in- and out-states of nuclear systems. Nucleons of momenta $Q \ll M_{Q C D}$ are thus associated in the EFT with an explicit field $N$, and nucleon propagation is represented by a line that goes through diagrams. The EFT splits into sectors of definite nucleon number $A$ : operators containing more than $2 A$ nucleon fields do not contribute to processes involving only $A$ incoming (and outgoing) nucleons. Because $Q \ll m_{N}$, an incoming slow nucleon receives only little kicks from other particles. Nucleons are thus non-relativistic, characterized in first approximation by a non-relativistic dispersion law for the energy, $E=$ $\vec{p}^{2} / 2 m_{N}$ as function of the three-momentum $\vec{p}$. Relativistic corrections are accounted for systematically in an expansion in $Q / m_{N}$.

$\S$ This can only be done because we include all allowed interactions. This is in sharp contrast to what is usually done in models like the Nambu-Jona-Lasinio model of low-energy QCD; such models are frequently called "effective", but they are not theories in the above sense. 
Which other hadronic states need to be included depends on what range of momenta we are interested in. Certainly all mesonic states with masses $m_{m} \gtrsim M_{Q C D}$ can be accounted for indirectly, through their contribution to coefficients of local interactions. In complete analogy with the multipole expansion in classical electrodynamics, we can approximate the interactions among nucleons originating from the exchange of these mesons in a series of contact interactions with an increasing number of derivatives. In first approximation such contact interactions are momentum-independent, and corrections generate an expansion in $Q / m_{m}$. Likewise, baryonic states with masses $m_{N^{*}} \gtrsim m_{N}+M_{Q C D}$ cannot be reached by hitting nucleons with small momenta. They can also be integrated out, generating local interactions in an expansion in $Q /\left(m_{N^{*}}-m_{N}\right)$.

The most relevant state besides the nucleon is the lightest hadron, the pion of mass $m_{\pi} \sim M_{\text {nuc }}$; next is the delta isobar, as its mass splitting to the nucleon is not large, $\delta m \equiv m_{\Delta}-m_{N} \sim M_{n u c}$. For $Q \lesssim M_{n u c}$ the pion and delta can be integrated out —unless we are considering external pions, in which case they could be treated as heavy particles as well. For $Q \gtrsim m_{\pi}$ the relativistic pion has to be included as an explicit field $\boldsymbol{\pi}$. For $Q \gtrsim m_{\Delta}-m_{N}$ a non-relativistic delta field $\Delta$ has to be considered as well. Because the two thresholds do not exactly coincide, it is possible - and possibly efficient - to integrate out the delta in a limited region of momenta above the pion threshold, while maintaining explicit pion fields. Coefficients of the corresponding effective Lagrangian that receive direct contributions from the delta will be relatively large, being suppressed only by powers of $Q / \delta$.

\section{Symmetries}

Symmetries play a fundamental role in EFTs because they restrict the possible form of interactions. The EFT corresponding to the standard electroweak theory at an energy scale of a few $\mathrm{GeV}$ involves only the lightest leptons and quarks, gluons, and the photon; weak gauge bosons, and heavy leptons and quarks can be integrated out in favor of nonrenormalizable interactions. Here I am interested mostly in interactions involving the lightest $u$ and $d$ quarks. They can be arranged in a flavor doublet $q=\left(\begin{array}{l}u \\ d\end{array}\right)$. Denoting by $G_{\mu}\left(A_{\mu}\right)$ the gluon (photon) field of strength $G_{\mu \nu}\left(F_{\mu \nu}\right)$, the relevant pieces of the effective Lagrangian at this scale are

$$
\begin{aligned}
\mathcal{L}= & -\bar{q}\left(\not \partial-i g_{s} G_{-}-i e Q A\right) q-\frac{1}{2}\left(m_{u}+m_{d}\right) \bar{q} q+\frac{1}{2}\left(m_{d}-m_{u}\right) \bar{q} \tau_{3} q \\
& -\frac{1}{2} \operatorname{Tr}\left[G_{\mu \nu} G^{\mu \nu}\right]+\frac{\bar{\theta} g_{s}^{2}}{32 \pi^{2}} \varepsilon_{\mu \nu \rho \sigma} \operatorname{Tr}\left[G^{\mu \nu} G^{\rho \sigma}\right]-\frac{1}{4} F_{\mu \nu} F^{\mu \nu}+\ldots
\end{aligned}
$$

Here $\tau_{3}$ is the usual Pauli matrix, $Q=1 / 6+\tau_{3} / 2$ is the quark charge matrix, and "..." denote non-renormalizable terms. Non-renormalizable interactions are suppressed by powers of the masses of the heavy particles that have been integrated out. I will neglect them in a (very good) first approximation. The leading Lagrangian (QCD + QED of quarks) has 5 parameters: the gauge couplings for strong $\left(g_{s}\right)$ and electromagnetic $(e)$ interactions, the masses of the up $\left(m_{u}\right)$ and the down $\left(m_{d}\right)$ quarks, and the strong CP parameter $(\bar{\theta})$. The theta term is found to be unnaturally small (the so-called strong CP problem), and I will neglect it as well. 
The set of remaining interactions in Eq. (9) has a number of approximate symmetries. They are clearly invariant under proper Lorentz transformations, parity, and time-reversal. Local changes of the quark-field phase that are proportional to unity or generators of the $S U(3)$ color group leave the Lagrangian invariant. A global $U(1)$ symmetry corresponds to baryon number, while another, axial $U(1)$ is anomalous. In the limit where $m_{u}, m_{d}$, and $e$ are zero there is further invariance under a chiral symmetry $S U(2) \times S U(2)$, consisting of independent rotations with parameters $\varepsilon_{L}$ and $\varepsilon_{R}$ of the left $\left(q_{L}=\left(1+\gamma_{5} / 2\right) q\right)$ and right $\left(q_{R}=\left(1-\gamma_{5} / 2\right) q\right)$ quark fields:

$$
\delta q_{L}=-i \varepsilon_{L} \cdot \boldsymbol{t} q_{L}, \quad \delta q_{R}=-i \varepsilon_{R} \cdot \boldsymbol{t} q_{R},
$$

where $\boldsymbol{t}=\boldsymbol{\tau} / 2$ are the generators of $S U(2)$ in terms of the Pauli matrices $\boldsymbol{\tau}$,

$$
t_{a} t_{b}=\frac{1}{4} \delta_{a b}+\frac{i}{2} \epsilon_{a b c} t_{c} .
$$

Acting on quark bilinears, $S U(2) \times S U(2) \sim S O(4)$. The absence of degenerate parity doublets but presence of (approximate) isospin multiplets in the hadron spectrum indicates that chiral symmetry is broken by the vacuum down to the diagonal subgroup $S U(2)_{V} \sim$ $S O(3)$ of isospin. Mass terms and electromagnetic interactions are not invariant under chiral transformations: they transform as non-trivial $S O(4)$ tensors and therefore break chiral symmetry explicitly. The operator $\bar{q} q$ breaks chiral symmetry as the fourth component of an $S O(4)$ vector

$$
S=\left(2 \bar{q} i \gamma_{5} \boldsymbol{t} q, \bar{q} q\right),
$$

while $-\bar{q} \tau_{3} q$ is the third component of another $S O(4)$ vector,

$$
P=\left(-2 \vec{q} \boldsymbol{t} q, \bar{q} i \gamma_{5} q\right) .
$$

Photon exchange among quarks produces four-quark interactions off which we can read the way electromagnetic interactions break isospin. Defining the $S O(4)$ antisymmetric tensor

$$
T^{\mu}=\left(\begin{array}{cc}
\varepsilon_{i j k} \bar{q} i \gamma^{\mu} \gamma_{5} t_{k} & \bar{q} i \gamma^{\mu} t_{i} q \\
-\bar{q} i \gamma^{\mu} t_{j} q & 0
\end{array}\right),
$$

one finds that chiral symmetry is explicitly broken by terms proportional to $T_{34}^{\mu}$ and $T_{34}^{\mu} T_{34}^{\nu}$.

The EFT has to produce the same low-energy $S$-matrix elements as the underlying theory. Although any choice of fields is equally valid, it is convenient to choose fields that transform under the above symmetries in the most transparent way. Although through field redefinitions other forms of interactions can be obtained where symmetries arise from a conspiracy of several terms, the particular choices of fields described next are the most convenient, as the pattern of symmetries is respected term-by-term in the resulting Lagrangian.

Spacetime symmetries. Parity and time-reversal can be implemented at low energies in the usual way, but proper Lorentz invariance deserves some care. Not surprisingly, the pion is represented by a relativistic pseudoscalar field with standard transformation properties, but heavy stable fields introduce a small complication. If I denote nucleons and deltas by 
a generic field $\hat{\psi}$ of mass $m \sim M$, then interactions will contain $\partial_{0} \hat{\psi} \sim-i m \hat{\psi}+\ldots$ and produce factors of $m / M$ that need to be resummed before we can develop an expansion in $Q / M$. On the other hand, the large energy $m$ is not really available for the dynamics, so we expect the result of the resummation to be well behaved. In fact, the result can be no other than a rearrangement of the non-relativistic expansion of the relativistic theory.

The resummation can be done from the start, avoiding the relativistic theory altogether, in what is referred to as the heavy-particle formalism [34,35]. We extract the rest energy by a field redefinition, $\hat{\psi} \equiv e^{-i m v \cdot x} \psi$ in a frame where the heavy particle moves with velocity $v$. The most general Lagrangian involving $\psi$ will be free of explicit factors of $m / M$ but will contain explicit factors of $v_{\mu}$. Proper Lorentz invariance requires that the Lagrangian be invariant under a boost to a frame of different velocity $w_{\mu}=v_{\mu}+q_{\mu} / m$, with $q \ll m$ satisfying $v \cdot q=-q^{2} / 2 m$. Fields in the two frames are related by a phase, besides the usual matrix $D(q)$ that accounts for the appropriate representation of $\psi$ under the Lorentz group: $\psi_{w}=e^{i q \cdot x} D(q) \psi_{v}$. Derivatives of $\psi$ transform in a more complicate way; as usual in such circumstances, it is profitable to introduce a covariant derivative

$$
\mathrm{d}_{\mu} \equiv \partial_{\mu}-i m v_{\mu}
$$

such that $\mathrm{d}_{\mu} \psi_{v}$ transform in the same way as $\psi_{v}$ itself. Out of $\mathrm{d}_{\mu} \psi$ and $\left(\mathrm{d}_{\mu} \psi\right)^{\dagger} \equiv \psi^{\dagger} \mathrm{d}_{\mu}^{\dagger}$ we can construct a covariant velocity and a symmetric derivative

$$
\mathcal{V}_{\mu} \equiv \frac{i}{2 m}\left(\mathrm{~d}_{\mu}-\mathrm{d}_{\mu}^{\dagger}\right), \quad \nabla_{\mu} \equiv \frac{1}{2 m}\left(\mathrm{~d}_{\mu}+\mathrm{d}_{\mu}^{\dagger}\right)
$$

which are useful building blocks, as in bilinears they produce Lorentz invariants. Spin can be dealt with in similar manner. If $\psi$ has spin $1 / 2$, then at low energy only two degrees of freedom are important, which can be enforced by imposing that the spinor $\psi_{v}$ obeys $(1-\not 0) \psi_{v}=0$. The covariant spin is

$$
\Sigma_{\mu}=\frac{i}{2} \gamma_{5} \sigma_{\mu \nu} \mathcal{V}^{\nu}
$$

as indeed $\Sigma \cdot \mathcal{V}=0$. If $\psi$ has spin $3 / 2$, then at low energy only four degrees of freedom are important; this can be enforced by demanding the field $\left(\psi_{v}\right)_{\mu}$ (which behaves as the tensor product of a Dirac spinor and a four-vector) obey $v \cdot \psi_{v}=0$ and $\gamma_{5} \sigma^{\mu \nu} v_{\mu}\left(\psi_{v}\right)_{\nu}=0$. To account for the vector character, the covariant spin-3/2 field has to be

$$
\left(\Psi_{v}\right)_{\mu}=\left(\psi_{v}\right)_{\mu}-\frac{i}{m} v_{\mu} \partial \cdot \psi_{v}
$$

Lorentz invariance is ensured by proper contraction of the indices of these covariant objects [35]. These invariants reduce in the rest frame of the heavy particle to a set of $S O(3)$ invariant operators, all with relative strengths determined by $1 / m$. The heavy-particle formalism is but a way to impose invariance under rotations plus slow velocity boosts; truncated at leading order in $1 / m$ it is just familiar Galilean invariance. As an example we can look at kinetic terms for $\psi$, i.e. terms containing only two heavy fields. Any such bilinear involving $\nabla_{\mu}$ can be eliminated in favor of total derivatives, so the most general kinetic term is of the form $\psi_{v}^{\dagger}\left(\mathcal{V}^{2}-1\right)^{n} \psi_{v}, n \geq 0$. By field redefinitions involving $\left(\mathcal{V}^{2}-1\right)$ 
we can eliminate all but $\psi_{v}^{\dagger}\left(i v \cdot \partial-\partial^{2} / 2 m\right) \psi_{v}$; by field redefinitions involving $i v \cdot \partial$ and $\partial^{2}$ we can systematically eliminate powers of $v \cdot \partial$ bigger than one. In the rest frame $v=(1, \overrightarrow{0})$ we then arrive at the standard

$$
\mathcal{L}=\psi^{\dagger}\left(i \partial_{0}+\frac{1}{2 m} \vec{\nabla}^{2}+\frac{1}{8 m^{3}} \vec{\nabla}^{4}+\ldots\right) \psi .
$$

When two heavy fields of mass $m$ and $m+\delta m, \delta m \ll M$, are considered in the same EFT, it is simpler to redefine both with the same phase $e^{i m v \cdot x}$. In this case the kinetic terms of the heavier field are as in Eq. (19) with additional terms $-\psi^{\dagger} \delta m(1+\ldots) \psi$.

A consequence is that we can alternatively work in the rest frame with heavy fields that provide definite representations of $S O(3)$ [15]. By field redefinitions we can systematically remove time-derivatives of the heavy fields from interactions. We take $N$ to be a Pauli spinor that provides the $1 / 2$ representation $\frac{1}{2} \vec{\sigma}$ of the spin generators,

$$
\sigma_{i} \sigma_{j}=\delta_{i j}+i \epsilon_{i j k} \sigma_{k}
$$

In the rest frame, $\psi=\left(\begin{array}{l}1 \\ 1\end{array}\right) N / \sqrt{2}$. Likewise, the $\Delta$ provides the $3 / 2$ representation of the spin generators. In the rest frame, $\psi_{\mu}=(0, \vec{S} \Delta)$,

$$
S_{i} S_{j}^{+}=\frac{1}{3}\left(2 \delta_{i j}-i \varepsilon_{i j k} \sigma_{k}\right)
$$

The $2 \times 4$ transition matrix $\vec{S}$ is used to couple $\Delta$ to $N$ in spin- 1 bilinears.

Internal symmetries. Goldstone's theorem [31] tells us that massless Goldstone bosons arise due to the assumed spontaneous breaking $S O(4) \rightarrow S O(3)$, and are naturally identified as pions. Pions are then associated with broken generators of $S O(4)$, and their fields live in the three-sphere $S O(4) / S O(3) \sim S^{3}$. We call the diameter of this sphere $F_{\pi}$; it is directly related to the chiral symmetry breaking scale, $\Lambda_{\chi S B}$, but the precise factor can only be obtained from the (so far elusive) mechanism of dynamical symmetry breaking in QCD. It turns out that this diameter can be determined from pion decay, and is the pion decay constant, $F_{\pi} \simeq 190 \mathrm{MeV}$. If we embed $S^{3}$ in the Euclidean $E^{4}$ space, $S O(4)$ transformations can be viewed as rotations in various $E^{4}$ hyperplanes. For example, $S U(2)_{V}$ of isospin consists of rotations in planes orthogonal to the fourth axis, while axial $S U(2)_{A}$ are rotations through planes that contain the fourth axis.

The sphere can be parametrized any way you want, say with four cartesian coordinates $\left\{\boldsymbol{\varphi}, \varphi_{4} \equiv \sigma\right\}$ subject to the constraint $\sigma^{2}+\varphi^{2}=F_{\pi}^{2} / 4$. The coordinates $\{\boldsymbol{\varphi}, \sigma\}$ transform linearly under $S O(4)$ rotations; for example, under an infinitesimal $S U(2)_{V}$ transformation with parameter $\varepsilon_{V}$,

$$
\delta_{V} \boldsymbol{\varphi}=\varepsilon_{V} \times \varphi
$$

It is more convenient, however, to work with three unconstrained coordinates $\boldsymbol{\pi}$ that can be directly associated with the pions. Any point on the sphere can be obtained by applying a four-rotation $R(\boldsymbol{\pi}), R(\boldsymbol{\pi}) R^{T}(\boldsymbol{\pi})=1$, to the north pole $\left(\mathbf{0}, \frac{1}{2} F_{\pi}\right)$ :

$$
\varphi_{\alpha}(\boldsymbol{\pi})=R_{\alpha 4}(\boldsymbol{\pi}) \frac{F_{\pi}}{2} .
$$


Different parametrizations of $R$ define different pion fields $\boldsymbol{\pi}$. The $\boldsymbol{\pi}$ 's just rotate,

$$
\delta_{V} \boldsymbol{\pi}=\varepsilon_{V} \times \boldsymbol{\pi}
$$

under $S U(2)_{V}$, but transform non-linearly under $S U(2)_{A}$. From a particular choice of $R(\boldsymbol{\pi})$ one can read the non-linear transformation of $\boldsymbol{\pi}$. The transformation law for $\partial_{\mu} \boldsymbol{\pi}$ is invariably complicated.

The general theory of non-linear realizations of a group is discussed in Ref. [36]. The main idea is to construct covariant objects that transform under the broken generators as under the unbroken subgroup, but with a field-dependent - thus local- parameter. The transformation we want to imitate is the rotation (22) and the appropriate field-dependent parameter for an axial transformation with parameter $\varepsilon_{A}$ is $\varepsilon_{A} \times \boldsymbol{\pi} / F_{\pi}$. We demand that the covariant derivative of the pion field

$$
\boldsymbol{D}_{\mu}=\frac{\partial_{\mu} \boldsymbol{\pi}}{F_{\pi}}\left(1+O\left(\boldsymbol{\pi}^{2} / F_{\pi}^{2}\right)\right)
$$

transform as an isospin-1 object,

$$
\delta_{V} \boldsymbol{D}_{\mu}=\varepsilon_{V} \times \boldsymbol{D}_{\mu}, \quad \delta_{A} \boldsymbol{D}_{\mu}=\left(\varepsilon_{A} \times \frac{\boldsymbol{\pi}}{F_{\pi}}\right) \times \boldsymbol{D}_{\mu} .
$$

Given the local look of $\delta_{A} \boldsymbol{D}_{\mu}$, it is not surprising that the covariant derivative of $\boldsymbol{D}_{\mu}$ is formed from a vector field

$$
\boldsymbol{E}_{\mu}=\frac{2 i}{F_{\pi}^{2}} \boldsymbol{\pi} \times \partial_{\mu} \boldsymbol{\pi}\left(1+O\left(\boldsymbol{\pi}^{2} / F_{\pi}^{2}\right)\right)
$$

as

$$
\mathcal{D}_{\mu} \boldsymbol{D}_{\nu}=\partial_{\mu} \boldsymbol{D}_{\nu}+i \boldsymbol{E}_{\mu} \times \boldsymbol{D}_{\nu}
$$

The pion fields being associated with broken generators, one can amass them in a matrix $u(\boldsymbol{\pi} \cdot \boldsymbol{t}) \equiv a\left(\boldsymbol{\pi}^{2}\right)+i b\left(\boldsymbol{\pi}^{2}\right) \boldsymbol{\pi} \cdot \boldsymbol{t}$ - with $a\left(\boldsymbol{\pi}^{2}\right)$ and $b\left(\boldsymbol{\pi}^{2}\right)$ two functions that depend on $R(\boldsymbol{\pi})$ defined so that

$$
\boldsymbol{D}_{\mu} \cdot \boldsymbol{t}=-\frac{i}{4}\left(u^{\dagger} \partial_{\mu} u-u \partial_{\mu} u^{\dagger}\right), \quad \boldsymbol{E}_{\mu} \cdot \boldsymbol{t}=\frac{1}{2}\left(u^{\dagger} \partial_{\mu} u+u \partial_{\mu} u^{\dagger}\right)
$$

For example, one can use stereographic coordinates [10] $\pi=2 \boldsymbol{\pi} /\left(1+2 \sigma / F_{\pi}\right)$, which correspond to

$$
R[\boldsymbol{\pi}]=\left(\begin{array}{cc}
\delta_{i j}-2 D^{-1} \frac{\pi_{i} \pi_{j}}{F_{\pi}^{2}} & 2 D^{-1} \frac{\pi_{i}}{F_{\pi}} \\
-2 D^{-1} \frac{\pi_{j}}{F_{\pi}} & D^{-1}\left(1-\frac{\boldsymbol{\pi}^{2}}{F_{\pi}^{2}}\right)
\end{array}\right)
$$

where

$$
D \equiv 1+\frac{\pi^{2}}{F_{\pi}^{2}}
$$

In this case, the pion covariant derivative and the vector field take particularly simple forms 


$$
\boldsymbol{D}_{\mu}=D^{-1} \frac{\partial_{\mu} \boldsymbol{\pi}}{F_{\pi}}, \quad \boldsymbol{E}_{\mu}=\frac{2 i}{F_{\pi}} \boldsymbol{\pi} \times \boldsymbol{D}_{\mu} .
$$

Other common choices of pion field $\boldsymbol{\pi}$ are the "sigma-model" parametrization

$$
u^{2}=\sqrt{1-\frac{4 \pi^{2}}{F_{\pi}^{2}}}+\frac{4 i}{F_{\pi}} \boldsymbol{\pi} \cdot \boldsymbol{t}
$$

and the "exponential" parametrization

$$
u=e^{\frac{2 i}{F_{\pi}} \pi \cdot \boldsymbol{t}} .
$$

Fields that couple to pions can be introduced easily once their isospin character is known. It simplifies to work with fields that transform under broken rotations with the same fielddependent parameter as before. In the case of a $(2 n+2)$-component field $\psi^{(n)}$ providing a representation $\boldsymbol{t}^{(n+1 / 2)}$ of isospin $n+1 / 2$,

$$
\delta_{V} \psi^{(n)}=i \varepsilon_{V} \cdot \boldsymbol{t}^{(n+1 / 2)} \psi^{(n)}, \quad \delta_{A} \psi^{(n)}=i\left(\boldsymbol{\varepsilon}_{A} \times \frac{\boldsymbol{\pi}}{F_{\pi}}\right) \cdot \boldsymbol{t}^{(n+1 / 2)} \psi^{(n)} .
$$

Its covariant derivative is then

$$
\mathcal{D}_{\mu} \psi^{(n)}=\left(\partial_{\mu}+\boldsymbol{t}^{(n+1 / 2)} \cdot \boldsymbol{E}_{\mu}\right) \psi^{(n)} .
$$

A nucleon field $N$ is a two-component isospinor and Eqs. (35) and (36) hold for $\psi^{(0)}=N$ and $\boldsymbol{t}^{(1 / 2)}=\boldsymbol{t}$. A delta field is a four-component spinor $\Delta$ and the same formulas apply for $\psi^{(1)}=\Delta$ and $\boldsymbol{t}^{(3 / 2)}$. Isospin-1 bilinears of $N$ and $\Delta$ can be formed using a $2 \times 4$ transition matrix $\boldsymbol{T}$ that satisfies

$$
T_{a} T_{b}^{+}=\frac{1}{6}\left(\delta_{a b}-i \varepsilon_{a b c} t_{c}\right)
$$

The introduction of fields that transform non-linearly makes it easy to construct chiral invariant objects: any isoscalar built out of the fields $\boldsymbol{D}_{\mu}$ and $\psi$, and their covariant derivatives will automatically be invariant under the whole $S U(2) \times S U(2)$ group. Operators $T_{\alpha \beta \ldots}\left[\boldsymbol{\pi} ; \boldsymbol{D}_{\mu}, \psi\right]$ that break chiral symmetry as tensor products of the tensors (12), (13), and (14) must also be constructed so that we can reproduce the correct symmetry pattern of low-energy $S$-matrix elements. Analogously to Eq. (23), where an $S O(4)$ vector involving the pion field $\boldsymbol{\pi}$ is constructed by applying a chiral rotation on another that does not, we just need to search for those tensors $T_{\alpha \beta \ldots}\left[\mathbf{0} ; \boldsymbol{D}_{\mu}, \psi\right]$ written in terms covariant objects only, then rotate them with $R(\boldsymbol{\pi})$ :

$$
T_{\alpha \beta \ldots}\left[\boldsymbol{\pi} ; \boldsymbol{D}_{\mu}, \psi\right]=\sum_{\alpha^{\prime} \beta^{\prime} \ldots} R_{\alpha \alpha^{\prime}}[\boldsymbol{\pi}] R_{\beta \beta^{\prime}}[\boldsymbol{\pi}] \ldots T_{\alpha^{\prime} \beta^{\prime} \ldots}\left[\mathbf{0} ; \boldsymbol{D}_{\mu}, \psi\right]
$$

For example, the simplest vector we can construct out of numbers only is $S_{(1)}[\mathbf{0} ; \mathbf{0}, 0]=(\mathbf{0}, 1)$ so from (38) and (30), the fourth component of

$$
S_{(1)}[\boldsymbol{\pi} ; \mathbf{0}, 0]=\left(2 D^{-1} \frac{\boldsymbol{\pi}}{F_{\pi}}, 1-2 D^{-1} \frac{\boldsymbol{\pi}^{2}}{F_{\pi}^{2}}\right)
$$


breaks chiral symmetry in exactly the same way as $\bar{q}$. By considering other vectors and tensors we can construct the infinite number of operators that break chiral symmetry as quark masses and electromagnetic interactions.

Because the EFT is formulated in terms of the lowest-lying color singlets, color gauge invariance plays no direct role. Soft photons (those with momenta $<M$ ) are not integrated out of the EFT and couple to pions, nucleons, and deltas in the most general way that respects $U(1)_{\text {em }}$ gauge invariance. The photon field $A_{\mu}$ enters through (i) "minimal substitution" in covariant derivatives,

$$
\partial_{\mu} \pi_{a} \rightarrow \partial_{\mu} \pi_{a}-e A_{\mu} \epsilon_{3 a b} \pi_{b}, \partial_{\mu} \psi^{(n)} \rightarrow \partial_{\mu} \psi-i e A_{\mu} Q_{\psi} \psi^{(n)},
$$

where $Q_{\psi}$ is the charge matrix of the field $\psi^{(n)}$ - for example, $Q_{N}=\left(1+\tau_{3}\right) / 2$; and (ii) gauge invariant objects constructed out of the field strength $F_{\mu \nu}$. There is, however, one subtlety regarding the role of gauge invariance: chiral symmetry is broken explicitly by the electromagnetic interaction because there is an anomaly in the third (isospin) component of the axial current. Terms have to be included in the effective Lagrangian in such a way that under a chiral transformation they reproduce the anomaly. This abnormal intrinsic parity sector is reviewed in Ref. [37]. Finally, further breaking of the above symmetries by the theta term [38] and non-renormalizable interactions [39] in the Lagrangian (9) can be included as above, mutatis mutandis.

\section{The power of counting}

Although symmetries restrict the number of interactions in the EFT, we still have to deal with an infinite number of them. The only hope of any predictive power relies on finding some ordering of contributions. Since it is not likely that the infinite number of coupling constants will be all small enough for a coupling-constant expansion to converge, we turn to the only obvious small quantity, the typical momentum $Q$ itself.

Powers of $Q$ of any particular Feynman diagram can be counted in ways analogous to the one used in finding the superficial degree of divergence (see, e.g., Ref. [40]). Each space derivative in an interaction produces a three-momentum in a vertex and therefore counts as $Q$. Likewise, each time derivative of a light field brings a $Q$ as well. In any loop, integration over the zeroth component of the four-momentum will generate two types of poles according to the scales appearing in the propagators: (i) standard poles at $\sim Q$ corresponding to external three-momenta and to the masses of the lightest excitations; (ii) shallow poles at $\sim Q^{2} / 2 m_{N}$ corresponding to initial energies.

Processes that involve at most one heavy particle line are the simplest because we can always close the contour avoiding shallow poles. As a consequence, each four-momentum integration brings a factor $Q^{4}$. A nucleon propagator is $Q^{-1}$ from the first term in the kinetic Lagrangian (19), the other terms being treated as corrections: the nucleon is a nearly static source propagating forward in time, as can be seen from the Fourier transform $\theta(t) \delta^{3}(\vec{r})+\ldots$ of its propagator. If $Q$ is sufficiently high so that pions and deltas are included explicitly, a pion propagator is $Q^{-2}$, and a delta propagator is $Q^{-1}$.

Processes that involve more than one stable heavy particle present a complication: a failure of perturbation theory that can potentially lead to bound states [10]. To see this, 


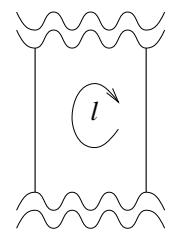

(a)

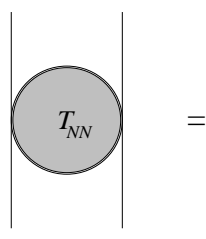

(b)

FIG. 1. (a) A two-nucleon intermediate state between two unspecified interactions, associated with a loop four-momentum $l$; (b) the full two-nucleon amplitude $T_{N N}$ as an iteration of a two-nucleon potential $V_{N N}$.

consider the scattering of two heavy particles with center-of-mass momentum $\vec{p}$ and total energy $E \equiv k^{2} / m+\ldots$. In-between two interactions, the heavy particles propagate forward in time. Such a piece of an arbitrary diagram is shown in Fig. 11(a). Neglecting smaller relativistic corrections, it will contribute

$$
\int \frac{d l^{0}}{2 \pi} \frac{1}{l^{0}+\frac{E}{2}-\frac{(\vec{l}+\vec{p})^{2}}{2 m_{N}}+i \epsilon} \frac{1}{-l^{0}+\frac{E}{2}-\frac{(\vec{l}+\vec{p})^{2}}{2 m_{N}}+i \epsilon}
$$

to the result of the diagram. The problem is that this integral is infrared enhanced, for it picks a large residue from one of the shallow poles. A naive momentum counting would have suggested a contribution of $O(1 / Q)$, yet we end up with something of $O\left(m_{N} / Q^{2}\right)$. This is quite a general phenomenon: such an enhancement of $O(M / Q)$ appears whenever two or more particles of mass $O(M)$ but momenta $O(Q)$ collide. As a consequence, for those contributions that come from shallow poles each four-momentum integration effectively brings a factor $Q^{5}$. A nucleon propagator is counted as $Q^{-2}$, and the first two terms in the kinetic Lagrangian (19) are important. A pion propagator still counts as $Q^{-2}$, but the pion can be taken in first approximation as static, and it is sometimes referred to as a "potential" pion. Contributions that come from standard poles naively scale as in processes with none or one heavy particle. Pions there are non-static or "radiative".

After integration over energies, each graph in covariant perturbation theory produces a number of terms. One can associate these with graphs in time-ordered perturbation theory, where loops represent three-momentum integrations and internal lines stand for denominators which contain energy differences between initial and intermediate states. In general, we can distinguish two classes of time-ordered diagrams. (Ir)reducible diagrams are those that can(not) be split in two pieces if in an intermediate state we cut all - and onlythe lines corresponding to initial or final particles; their denominators will (not) contain energy differences of $O\left(Q^{2} / m_{N}\right)$.

In a system of heavy particles, we call the sum of irreducible diagrams the potential $V$. Note that the potential being a set of subgraphs, it can be defined in alternative ways. All definitions that exclude the infrared enhancement contributions but differ by a smaller amount are equally good, as long as no double-counting or omissions are made. The important point is that the only scale appearing explicitly in the potential is $Q$, so that the power counting proceeds as in the case of diagrams with at most one heavy particle. 
Reducible diagrams can be obtained by sewing together irreducible diagrams. The full amplitude $T$ is a sum of the potential and its iterations; schematically, with $G_{0}$ denoting the intermediate state containing only initial or final particles,

$$
T=-\left(V+V G_{0} V+V G_{0} V G_{0} V+\ldots\right)=-V+V G_{0} T .
$$

This is just the Lippmann-Schwinger equation, which is formally equivalent to the Schrödinger equation with the potential $V$. Fig. 1 (b) illustrates the case of two nucleons, which formally evolve according to the familiar non-relativistic Schrödinger propagator

$$
G_{0}(l ; k)=-\frac{m}{l^{2}-k^{2}-i \epsilon}, \quad G_{0}(r ; k)=-m \frac{e^{i k r}}{4 \pi r} .
$$

Relativistic corrections are accounted for by a two-legged vertex

$$
u(l ; k)=-\frac{l^{4}-k^{4}}{8 m^{3}}+\ldots,
$$

and can be lumped in the potential.

With these ingredients one can write the contribution of any diagram to the amplitude as

$$
T \propto Q^{\nu} \mathcal{F}(Q / \Lambda)
$$

where $\Lambda$ is a renormalization scale, and $\nu$ is a counting index. The exact formula for $\nu$ in terms of the elements of a diagram depends on the $Q$ we are interested in. In addition, electromagnetic interactions can be considered through a simultaneous expansion in $\alpha=e^{2} / 4 \pi$. We will discuss the power counting in more detail below.

Counting powers of $Q$ is not in itself sufficient for an ordering of interactions. We need to find which other scales accompany $Q$. As we have mentioned in the introduction, there are at least three physical scales in the problem: $\aleph, M_{n u c}$, and $M_{Q C D}$. The largest possible ratio is $Q / \aleph$. From dimensional analysis alone, then, we expect that for $Q \ll \aleph$ amplitudes for scattering of free nucleons will be perturbative in $Q / \aleph$. Such systems are labeled dilute. As $Q$ increases, we have to perform resummations: first summing all orders in $Q / \aleph$ but expanding in $Q / M_{n u c}$; then summing all orders in $Q / M_{n u c}$ but expanding in $Q / M_{Q C D}$; and so on. As we resum certain class of diagrams, bound-states may appear.

Given that we do not yet possess a full solution of QCD, it is obvious that some assumptions have to be made about the parameters of the EFT. The minimal assumption is that of naturalness [41]. Naturalness means simply that for each operator $\mathcal{O}_{i}$ of dimension $\delta_{i}$ in Eq. (6), the parameter $g_{i}(\Lambda)$ will be of form $c_{i}(\Lambda / M) / M^{\delta_{i}}$ with $c_{i}(1)=O(1)$ ***, unless there exists a symmetry that forces $c_{i}$ to be small. As we are going to see, the presence in the nuclear problem of scales lighter than $M_{Q C D}$ requires slightly stronger assumptions about the scaling of the EFT parameters. These new assumptions are still fairly general and are

\footnotetext{
** Using a mass cutoff $\Lambda \ll M$ introduces a large dimensionless ratio $M / \Lambda$ and can unnecessarily complicate the power counting.
} 
motivated by qualitative features of nuclear phenomenology; they are much less restrictive than usual model assumptions. In any case, these assumptions are necessary for the a priori ordering of contributions, but they are subject to test a posteriori. If a parameter is found much larger than assumed initially, the EFT can be improved to better account for its effects.

\section{E. Propaganda}

The EFT approach is:

(i) systematic. Once a power counting is established, there is an expansion in a small parameter $\propto Q$.

(ii) consistent with QCD. The only (very important) QCD inputs are confinement, (chiral, ...) symmetries, and some version of naturalness. QCD can be represented by a point in the space of renormalized parameters (at some renormalization scale) of the effective theory. An explicit solution of QCD (such as from simulations on a lattice) would provide knowledge of the exact position of this point, and then the EFT would be completely predictive. Until such a solution is found - or as a test of QCD after it is found - we can recourse to fitting of low-energy experiments in order to determine the region in parameter space allowed on phenomenological grounds. Even in this case the theory is predictive, because to any given order the space of parameters is finite-dimensional. After a finite number of experimental results are used, an infinite number of others can be predicted up to an accuracy depending on the order of the expansion. In practice, because the number of parameters grows rapidly with the order, model-dependent estimates of parameters based on specific dynamical ideas - such as saturation by tree-level resonance exchange [12] - are sometimes used.

(iii) a consistent quantum field theory. Renormalization can be carried out to relate parameters appearing in different processes and to remove cutoff dependence at each order in the expansion. As a consequence, it is applicable in principle to all low-energy phenomena. The literature on applications to mesons and one-nucleon processes is vast; after a lightning review I will show how we can also gain insight into nuclear physics per se.

\section{ONE- OR NO-NUCLEON PROCESSES}

The EFT at momenta $Q \ll M_{n u c}$ contains only nucleons, pions treated as heavy particles, and photons. All interactions are of contact type, and form an expansion in $Q / M_{n u c}$. The relevant symmetries are Lorentz and $U(1)_{\mathrm{em}}$ local invariance. Processes involving two or more pions, or one nucleon and at least one pion are of the type considered in the next sections. Processes involving one pion or one nucleon and a number of photons give rise to the various well-known electromagnetic parameters. Particle form factors take a multipole form, that is, an expansion in charge and anomalous magnetic moment, radii, etc. Compton scattering has a Thomson-seagull interaction and a magnetic moment piece, followed by polarizabilities, and so on. The analytic nature of these expansions in $Q / M_{n u c}$ and the limited symmetry constraints make this EFT not very interesting. We expect, of course, that a sizable fraction of the value of the parameters comes from the lightest state that was 
integrated out, the pion. As $Q$ is increased, the approximation of zero-range propagation for pions becomes less and less reliable.

At momenta $Q \sim m_{\pi}$, pions have to be included explicitly in the theory. Because numerically $\delta m$ is $\sim 2 m_{\pi}$, convergence of the EFT for $Q \sim M_{n u c}$ should be optimized by the concomitant inclusion of an explicit delta degree of freedom. Now, we are in luck because pion interactions are not arbitrary. Once explicit pion fields are considered, approximate chiral symmetry imposes important restrictions on the way pions couple among themselves and to other degrees of freedom. The delta, too, can be included without too much hassle, since at these momenta it is, like the nucleon, a non-relativistic object, to which we can apply the heavy-particle formalism. In the preceding section all the ingredients to construct this EFT were presented.

The power counting (45) presents no problems as the only explicit scales are $M_{n u c}$ and $Q$, contributions from momenta of order $M_{Q C D}$ being lumped in the vertices. Assuming $Q \sim M_{n u c}$, it follows that for a diagram involving $A=0,1$ nucleon and any number of pions

$$
\nu=2-A+2 L+\sum_{i} V_{i} \Delta_{i}, \quad \Delta_{i} \equiv d_{i}+f_{i} / 2-2,
$$

where $L$ is the number of loops and $V_{i}$ is the number of vertices of type $i$, which contains $d_{i}$ derivatives or powers of $m_{\pi}$ and $f_{i}$ fermion fields [6,10]. This formula is important because chiral symmetry places a lower bound on the interaction index $\Delta_{i} \geq 0$. Since $L$ is bounded from below $(L \geq 0), \nu \geq \nu_{\text {min }}=2-A$ for strong interactions. An expansion in $Q / M_{Q C D}$ results. It starts at $\nu=\nu_{\text {min }}$ with tree $(L=0)$ diagrams built out of vertices of index 0 $\left(\sum V_{i} \Delta_{i}=0\right)$, then proceeds at $\nu=\nu_{\text {min }}+1$ with further tree diagrams, now with one vertex of index 1 , the remaining having index $0\left(\sum V_{i} \Delta_{i}=1\right)$. These first two orders are equivalent to the current algebra of the 60's, but now unitarity corrections can be accounted for systematically. At $\nu=\nu_{\min }+2$, for example, besides tree diagrams with one index2 interaction or two index-1 interactions $\left(\sum V_{i} \Delta_{i}=2\right)$, there are also one-loop $(L=1)$ diagrams built out of index-0 vertices $\left(\sum V_{i} \Delta_{i}=0\right)$. This is generalized to higher orders in obvious fashion. We discuss isospin breaking soon.

It is convenient then to split the chiral Lagrangian in pieces $\mathcal{L}^{(\Delta)}$ labeled by the index $\Delta$. For example, the lower-order Lagrangians are 15, 16, 43, 44

$$
\begin{gathered}
\mathcal{L}^{(0)}=-\frac{F_{\pi}^{2}}{2} \boldsymbol{D}^{2}-\frac{1}{2} m_{\pi}^{2} D^{-1} \boldsymbol{\pi}^{2}+N^{\dagger} i \mathcal{D}_{0} N-2 g_{A} \boldsymbol{t} \cdot \vec{\sigma} \cdot \overrightarrow{\boldsymbol{D}} N \\
+\Delta^{\dagger}\left(i \mathcal{D}_{0}-\delta m\right) \Delta-2 h_{A}\left[N^{\dagger} \boldsymbol{T} \cdot \vec{S} \cdot \overrightarrow{\boldsymbol{D}} \Delta+h . c .\right]+\ldots, \\
\mathcal{L}^{(1)}=\frac{1}{2 m_{N}} N^{\dagger} \overrightarrow{\mathcal{D}}^{2} N-\frac{g_{A}}{m_{N} F_{\pi}}\left[i N^{\dagger} \boldsymbol{t} \cdot \boldsymbol{D}_{0} \vec{\sigma} \cdot \overrightarrow{\mathcal{D}} N+h . c .\right] \\
+\frac{1}{2 m_{N}} \Delta^{\dagger} \overrightarrow{\mathcal{D}}^{2} \Delta-\frac{h_{A}}{m_{N} F_{\pi}}\left[i N^{\dagger} \boldsymbol{T} \cdot \boldsymbol{D}_{0} \vec{S} \cdot \overrightarrow{\mathcal{D}} \Delta+h . c .\right] \\
-B_{1} \boldsymbol{D}^{2} N^{\dagger} N-B_{2}(\overrightarrow{\boldsymbol{D}} \times \overrightarrow{\boldsymbol{D}}) \cdot N^{\dagger} \boldsymbol{t} \vec{\sigma} N-\frac{B_{3} m_{\pi}^{2}}{F_{\pi}^{2}} D^{-1} \boldsymbol{\pi}^{2} N^{\dagger} N-B_{4} \boldsymbol{D}_{0}^{2} N^{\dagger} N+\ldots
\end{gathered}
$$

Here $g_{A}, h_{A}=O(1)$ and $B_{i}=O\left(1 / M_{Q C D}\right)$ are undetermined constants, to be obtained either by solving QCD or by fitting data; “..." stand for other terms involving the delta isobar 
which do not appear explicitly below. Higher-index interactions can be constructed similarly Note that pion self-interactions appear only at even index because of Lorentz invariance.

The most general isospin-violating Lagrangian [15,18] is constructed out of operators that break isospin in three different ways. First, there are gauge invariant couplings to soft photons. For example, from minimal substitution in the index-0 Lagrangian,

$$
\begin{aligned}
\mathcal{L}_{s p}^{(-1)}= & -e A^{\mu}\left(\boldsymbol{\pi} \times \partial_{\mu} \boldsymbol{\pi}\right)_{3}+\frac{1}{2} e^{2} A^{2}\left(\boldsymbol{\pi}^{2}-\pi_{3}^{2}\right) \\
& -e A_{0} N^{\dagger}\left(\frac{1}{2}+t_{3}\right) N+\frac{2 e g_{A}}{F_{\pi}} \vec{A} \cdot N^{\dagger} \vec{\sigma}(\boldsymbol{t} \times \boldsymbol{\pi})_{3} N+\ldots
\end{aligned}
$$

Second, there are operators that transform as products of the quark-mass-difference term; they are proportional to $\varepsilon m_{\pi}^{2}$, where $\varepsilon \equiv m_{d}-m_{u} /\left(m_{d}+m_{u}\right) \sim 1 / 3$ 445. For example,

$$
\begin{gathered}
\mathcal{L}_{q m}^{(1)}=\delta m_{N}\left(-N^{\dagger} t_{3} N+2 D^{-1} \frac{\pi_{3} \boldsymbol{\pi}}{F_{\pi}^{2}} \cdot N^{\dagger} \boldsymbol{t} N\right)+\ldots, \\
\mathcal{L}_{q m}^{(2)}=\frac{1}{2} D^{-2} \delta m_{\pi}^{2} \pi_{3}^{2}+\beta_{1}\left(\vec{D}_{3}-\frac{2 D^{-1}}{F_{\pi}^{2}} \pi_{3} \boldsymbol{\pi} \cdot \overrightarrow{\boldsymbol{D}}\right) \cdot N^{\dagger} \vec{\sigma} N+\ldots,
\end{gathered}
$$

with $\delta m_{N}=O\left(\varepsilon m_{\pi}^{2} / M_{Q C D}\right), \delta m_{\pi}^{2}=O\left(\varepsilon^{2} m_{\pi}^{4} / M_{Q C D}^{2}\right)$, and $\beta_{1}=O\left(\varepsilon m_{\pi}^{2} / M_{Q C D}^{2}\right)$. Third, there are operators that transform as four-quark interactions generated by hard-photon exchange, which are proportional to $\alpha$. For example,

$$
\begin{gathered}
\mathcal{L}_{h p}^{(-2)}=-\frac{1}{2} D^{-2} \bar{\delta} m_{\pi}^{2}\left(\boldsymbol{\pi}^{2}-\pi_{3}^{2}\right) \\
\mathcal{L}_{h p}^{(-1)}=\bar{\delta} m_{N}\left[-N^{\dagger} t_{3} N+\frac{2 D^{-1}}{F_{\pi}^{2}} N^{\dagger}\left(\boldsymbol{\pi}^{2} t_{3}-\pi_{3} \boldsymbol{\pi} \cdot \boldsymbol{t}\right) N\right]+\ldots
\end{gathered}
$$

with $\bar{\delta} m_{\pi}^{2} \propto \alpha M_{Q C D}^{2}$ and $\bar{\delta} m_{N}=O\left(\bar{\delta} m_{\pi}^{2} / M_{Q C D}\right)$.

The observed smallness of isospin violation at low energies is not immediately evident within QCD, as chiral symmetry breaking effects are not negligible, and $\varepsilon$ is not particularly small. Power counting offers an explanation for this phenomenon [15, 18]. The first task is to compare the different towers of isospin-violating operators. This can be accomplished by noticing that photon loops (containing two electromagnetic interactions) typically produce factors of $\alpha / \pi$, which is numerically $\sim \varepsilon\left(m_{\pi} / m_{\rho}\right)^{3}$. This immediately suggests that $\mathcal{L}_{h p}^{(n)} \sim$ $\mathcal{L}_{q m}^{(n+3)}$ and $\mathcal{L}_{s p}^{(n)} \sim \mathcal{L}_{q m}^{\left(n+\frac{3}{2}\right)}$. It can easily be verified that this estimate produces the right order of magnitude for the various contributions to the pion and nucleon mass differences, $\Delta m_{\pi}^{2}=\bar{\delta} m_{\pi}^{2}+\delta m_{\pi}^{2}+\ldots$ and $\Delta m_{N}=\delta m_{N}+\bar{\delta} m_{N}+\ldots$, respectively. One now can see that, because there is no lowest-order isospin-violating interactions, isospin-violating quantities are typically not only $O(\varepsilon)$ smaller than chiral-violating quantities, but are in general further suppressed by factors of $Q / M_{Q C D}$ : isospin is an accidental symmetry 15,18. For simplicity, when considering only (a fixed number of) external photons, I will count a power of $e$ as a contribution of 1 to the index $\Delta$ in Eq. (46). 
Because $\delta m \sim M_{n u c}$, delta effects appear for $Q \sim M_{n u c}$ typically at the same order as nucleon contributions . At momenta $Q<\delta m$ we can integrate out the delta, producing an EFT with nucleons and pions only. This entails significant reduction in the number of interactions, as the delta terms disappear and the remaining interactions among nucleons and pions have the same structure as those in the $\mathcal{L}^{(\Delta)}$ 's above. Only their coefficients change, and now contain $O(1 / \delta m)$ terms. For example, $\mathcal{L}^{(1)}$ has the same pion-nucleon seagulls with coefficients traditionally called $c_{i}$ in the literature [44] and related to the $B_{i}$ via

$$
\begin{gathered}
c_{1}=\frac{B_{3}}{8}+\ldots, \quad c_{2}=-\frac{1}{4}\left(B_{4}-\frac{g_{A}^{2}}{2 m_{N}}\right)+\frac{h_{A}^{2}}{9 \delta m}+\ldots, \\
c_{3}=\frac{B_{1}}{4}-\frac{h_{A}^{2}}{9 \delta m}+\ldots, \quad c_{4}=\frac{1}{4}\left(B_{2}-\frac{1}{m_{N}}\right)+\frac{h_{A}^{2}}{18 \delta m}+\ldots
\end{gathered}
$$

This machinery can be applied to soft processes involving any number of pions and photons, and at most one nucleon. $\chi \mathrm{PT}$ is particularly relevant for those processes where the pion plays a dominant role. Here I briefly present some of the highlights that are directly relevant for what follows. For details on pion processes see, for example, Ref. [46]; one-nucleon processes in the deltaless theory have been extensively reviewed in Ref. [44, and explicit delta effects are discussed in Ref. [47].

Pion-nucleon scattering is perhaps the most important process where the chiral Lagrangian can be applied, as it can be used to test the fermionic sector of the theory and determine some of its parameters. Contributions start at $\nu=\nu_{\min }=1$ from Born graphs with intermediate nucleons and deltas, and from the $\pi \pi N N$ interaction ("Weinberg-Tomozawa seagull") generated by the chiral covariant derivative in the kinetic nucleon term of Eq. (47). At $\nu=\nu_{\min }+1$ the $B_{i}$-seagull diagrams contribute at tree level. Dropping nucleonic Born terms, the $\pi_{a} N \rightarrow \pi_{b} N$ amplitude is traditionally written [48] as

$$
t_{a b} \propto \delta_{a b}\left[a+b \vec{q} \cdot \vec{q}^{\prime}+c\left(\vec{q}^{2}+\vec{q}^{\prime 2}\right)+e \omega \omega^{\prime}\right]-d \epsilon_{a b c} \tau_{c} \vec{\sigma} \cdot \vec{q} \times \vec{q}^{\prime}+\ldots
$$

where $\vec{q}\left(\vec{q}^{\prime}\right)$ is the incoming (outgoing) pion momentum and $\omega\left(\omega^{\prime}\right)$ is the initial (final) pion energy. The $a, \ldots, e$ coefficients are given in terms of the $c_{i}$ 's as

$$
a=\frac{16 m_{\pi}^{2} c_{1}}{F_{\pi}^{2}}, \quad b=\frac{8 c_{3}}{F_{\pi}^{2}}, \quad c=0, \quad d=-\frac{4 c_{4}}{F_{\pi}^{2}}, \quad e=-\frac{8\left(c_{2}+c_{3}\right)}{F_{\pi}^{2}} .
$$

At $\nu=\nu_{\text {min }}+2$ a host of terms enter, including loop diagrams and new parameters. Very close to threshold, where only $S$ waves matter, chiral symmetry predicts an isovector scattering length $b^{(1)} \sim m_{\pi} / F_{\pi}^{2}$ from the Weinberg-Tomozawa seagull [49] and a smaller isoscalar

\footnotetext{
${ }^{\dagger \dagger}$ Some people reserve the name $\chi \mathrm{PT}$ to the EFT of the world where $Q / \delta m \sim m_{\pi} / \delta m \ll 1$, and refer to the EFT of a world where $Q / \delta m \sim m_{\pi} / \delta m \sim 1$ as the "small scale expansion". I find it simpler to refer to them as deltaless and deltaful $\chi \mathrm{PT}$, respectively.
} 
scattering length $b^{(0)} \sim m_{\pi}^{2} / F_{\pi}^{2} M_{Q C D}$, related to the sigma-term, from the subleading $c_{i}$ seagulls. The most extensive calculations have been performed to $\nu=\nu_{\min }+2$ in deltaless $\chi \mathrm{PT}$. Different pieces of $\pi N$ scattering data have been used to determine the chiral coefficients. In lowest order, $g_{A} \simeq 1.25$; in sub-subleading order chiral symmetry breaking corrections appear, and if they are absorbed in $g_{A}$, then $g_{A} \simeq 1.32$ satisfies the Goldberger-Treiman

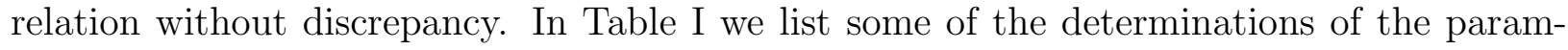
eters $c_{i}$. Earlier fits [44,50 52] were made to different sets of threshold and sub-threshold parameters obtained from dispersion analyses of older data. Newer fits [53] were made to different phase-shift analyses (PSAs), the last two in Table [ including the more modern meson-factory data. The $O\left(Q^{3}\right)$ determinations are consistent with each other when their error bars (not shown) are considered, except for $c_{1}$, which reflects the higher value for the sigma-term in the newer PSAs. Note that the coefficients $c_{2}, c_{3}$, and $c_{4}$, which receive contributions from the $\Delta$ at tree level, are larger than $c_{1}$, as expected. There are not yet very reliable determinations of $h_{A}$ from $\pi N$ scattering in the deltaful theory. The large- $\mathcal{N}_{c}$-limit value, $h_{A}=3 g_{A} / \sqrt{2} \simeq 2.7$, gives a reasonable delta decay width at tree level.

The only cases where isospin violation can be large are instances where the leading isospin-conserving interactions do not contribute. In such cases we can encounter an isospin violation of size comparable to $\varepsilon$. One example is $S$-wave $\pi N$ scattering [45, 15, 18, [4]]. The leading isospin-violating term involving a nucleon is, according to the above discussion, given by Eq. (50). It determines not only the most important quark-mass contribution $\delta m_{N}$ to the nucleon mass splitting, but also isospin-breaking interactions of an even number of pions with a nucleon. It contributes $\sim \delta m_{N} / F_{\pi}^{2}$ to the pion-nucleon scattering lengths. This gives a small contribution $\sim \delta m_{N} / m_{\pi} \sim \varepsilon m_{\pi} / M_{Q C D} \sim 5 \%$ to processes that involve at most one $\pi^{0}$, as they receive a leading-order contribution from the Weinberg-Tomozawa seagull. But for $\pi^{0} N \rightarrow \pi^{0} N$, which starts at subleading order with the isoscalar seagulls, isospin violation can be as large as $\varepsilon \sim 30 \%$, thus revealing the full isospin breaking in the QCD Lagrangian! Unfortunately, this one case of large isospin violation cannot be measured easily.

Pion photoproduction at threshold, where the pion is mostly in an $S$ wave, has received a lot of attention lately from both theoretical and experimental standpoints. This process has been studied near threshold up to $\nu=\nu_{\min }+3$ in the deltaless theory [55]. At leading

TABLE I. Subleading $\chi \mathrm{PT}$ coefficients in $\mathrm{GeV}^{-1}$ from several recent fits.

\begin{tabular}{|c|ccccc|}
\hline \hline Fit & $c_{1}$ & $c_{2}$ & $c_{3}$ & $c_{4}$ \\
\hline$O\left(Q^{2}\right)$ & {$[50$} & 1.78 & -3.90 & 2.25 \\
$O\left(Q^{3}\right)$ & -0.64 & 3.30 & -5.25 & 4.12 \\
$O\left(Q^{3}\right)$ & -0.87 & 3.34 & -5.29 & 3.63 \\
$O\left(Q^{3}\right)$ & -0.93 & 3.40 & -5.54 & 3.25 \\
$O\left(Q^{3}\right)$ & -1.06 & 3.23 & -5.93 & 3.44 \\
$O\left(Q^{3}\right)$ & -1.27 & 3.21 & -6.00 & 3.52 \\
$O\left(Q^{3}\right)$ & -1.47 & 3.22 & -6.19 & 3.51 \\
\hline \hline
\end{tabular}


order, $\nu=\nu_{\min }=1$, there exist only contributions to charged channels from the fourth term in Eq. (49) ("Kroll-Ruderman term") and from the pion pole; we thus expect the charged channels to be larger. At $\nu=\nu_{\min }+1$, minimal substitution on the recoil correction to the pion-nucleon vertex contributes to photoproduction on the proton. At $\nu=\nu_{\min }+2$ there are tree-level contributions involving the magnetic moment of the nucleon. Together with the previous orders, this reproduces an old "low-energy theorem". However, still at $\nu=\nu_{\min }+2$ there are large loop corrections where the photon interacts with a virtual pion which then rescatters on the nucleon before flying away to the detector. This quantum effect was missed in the old "low-energy theorem" and in most models. It is determined in terms of known parameters and thus constitutes a new, corrected low-energy theorem. At $\nu=\nu_{\min }+3$ other loops and new counterterms appear. Results for the cross-section at threshold up to $\nu=\nu_{\min }+3$ from a fit to data above threshold constrained by resonance saturation of parameters [55] are satisfactory. To this order there is a prediction for the near threshold behavior of the $\gamma n \rightarrow \pi^{0} n$ reaction, which would be important to test.

Compton scattering on the nucleon can also be calculated with this EFT. To $\nu=\nu_{\min }+2$, the amplitude contains non-analytic terms and is completely predictive [44]. Expanded for small energies, it yields predictions for the polarizabilities, which are determined by the pion cloud. There is good agreement with data on the proton, and again it would be important to test the corresponding neutron predictions.

We have seen that there are a number of tantalizing predictions from $\chi \mathrm{PT}$ concerning nucleon structure, particularly involving the neutron and neutral pions. These are hard to test because neutron targets and neutral pion beams do not come by easily... Nuclear targets and virtual pion effects can compensate for this, if we can formulate an EFT for nuclear systems that is consistent with $\chi \mathrm{PT}$. We are thus naturally led to nuclear physics proper.

\section{THE TWO-NUCLEON SYSTEM}

The two-nucleon system is where we hope to understand the basic modifications in the $\chi \mathrm{PT}$ power counting needed to tackle bound states. I will in turn consider the three regions of momenta that have been investigated: $Q<M_{n u c}$ in Subsect. IVA, $Q<M_{N N}$ in Subsect. IVB, and $Q<M_{Q C D}$ in Subsect. IVG, although this is not a historical order.

\section{A. Very low energies}

The lowest range of momenta serves to illustrate the method of EFT in the simplest non-perturbative context. Because only nucleons appear explicitly, we can go a long way following analytical arguments, while a good fraction of the physics of higher momenta is just a generalization of the same ideas to a more complicated situation. $S$-matrix elements for $N N$ scattering at momenta $Q \ll M_{n u c}$ can be obtained in zeroth order in $\alpha$ from an effective Lagrangian involving arbitrarily complicated operators of only $N$ and its derivatives. The only symmetry constraints come from Lorentz transformations of small velocity, parity and time-reversal. Electromagnetic interactions can be included by adding all possible $U(1)_{\mathrm{em}^{-}}$ invariant terms. In the two-nucleon system it will give rise to photon exchange in addition 
to dressings already present in one-nucleon systems. This will be discussed after we consider strong interactions in the $\alpha \rightarrow 0$ limit.

The amplitude. The issue of establishing a power counting is largely independent of complications generated by spin and isospin, so for now consider [56] a system of two identical, stable spinless particles $\psi$ in their center-of-mass frame at energy $E=k^{2} / m-$ $k^{4} / 4 m^{3}+\ldots$ much smaller than their mass $m$, their internal excitation energy, and the range of their interaction, which I will denote collectively by $M$. In addition to the kinetic terms in Eq. (19), we have to consider the most general Lagrangian involving four nucleon fields, which can be written as [56]

$$
\begin{aligned}
\mathcal{L}= & -\frac{1}{2} C_{0} \psi^{\dagger} \psi \psi^{\dagger} \psi \\
& -\frac{1}{8}\left(C_{2}+C_{2}^{\prime}\right)\left[\psi^{\dagger}(\vec{\nabla}-\overleftarrow{\nabla}) \psi \cdot \psi^{\dagger}(\vec{\nabla}-\overleftarrow{\nabla}) \psi-\psi^{\dagger} \psi \psi^{\dagger}(\vec{\nabla}-\overleftarrow{\nabla})^{2} \psi\right] \\
& +\frac{1}{4}\left(C_{2}-C_{2}^{\prime}\right) \psi^{\dagger} \psi \vec{\nabla}^{2}\left(\psi^{\dagger} \psi\right)+\ldots
\end{aligned}
$$

where the $C_{2 n}$ 's are parameters of mass dimension $-2(1+n)$ that depend on the details of the dynamics of range $\sim 1 / M$. The "..." stand for operators with more derivatives.

Conservation of particle number reduces the $T$-matrix to a sum of bubble diagrams. The potential is given in momentum space by

$$
V\left(p, p^{\prime}\right)=C_{0}+C_{2}\left(\vec{p}^{2}+\vec{p}^{\prime 2}\right)+2 C_{2}^{\prime} \vec{p} \cdot \vec{p}^{\prime}+\ldots,
$$

$\vec{p}\left(\vec{p}^{\prime}\right)$ being the relative momentum of the incoming (outgoing) particles. If Fouriertransformed to coordinate space, these bare interactions consist of delta functions and their derivatives. The bubbles require regularization and renormalization, which introduce a mass scale $\Lambda$ through some cutoff function $F\left(l^{2} / \Lambda^{2}\right)$. A bubble involving $n \geq 0$ derivatives at the vertices gives

$$
\begin{aligned}
I_{2 n}(k) & =\int \frac{d^{3} l}{(2 \pi)^{3}} l^{2 n} G_{0}(l ; k) F\left(l^{2} / \Lambda^{2}\right) \\
& =-\frac{m}{2 \pi^{2}}\left[\sum_{i=0}^{n} k^{2 i} \theta_{2(n-i)+1} \Lambda^{2(n-i)+1}+i \frac{\pi}{2} k^{2 n+1}+\frac{k^{2(n+1)}}{\Lambda} R\left(k^{2} / \Lambda^{2}\right)\right],
\end{aligned}
$$

where $\theta_{n}$ is a number that depends on the regularization scheme chosen, and $R(x)$ is a regularization-scheme-dependent function that approaches a finite limit as $x \rightarrow 0$. In general $\theta_{n}$ and $R(x)$ can be non-vanishing with either sign. The severe $\Lambda$-dependence comes from the region of high momenta that cannot be described correctly by the EFT; it can be removed by lumping these terms together with the unknown bare parameters into "renormalized" coefficients $C_{2 n}^{(R)}$. Only the latter are observable. The regulator-independent, non-analytic piece in Eq. (59), on the other hand, is characteristic of loops: it cannot be mocked up by

\footnotetext{
$\ddagger$ Although I refer to this as a cutoff, I am not assuming any particular regularization scheme.
} 
re-shuffling contact interactions. From Eq. (59) we see that we can estimate its effects by associating a factor $m Q / 4 \pi$ to each loop and a factor $Q$ to each derivative at the vertices.

The $T$-matrix is given by the diagrams in Fig. 2. For the first few terms one finds on-shell

$$
\begin{aligned}
T_{\text {os }}\left(k, \hat{p}^{\prime} \cdot \hat{p}\right)=-C_{0}^{(R)}\{ & 1-\left(\frac{m C_{0}^{(R)}}{4 \pi} i k\right)+\left(\frac{m C_{0}^{(R)}}{4 \pi} i k\right)^{2}-\left(\frac{m C_{0}^{(R)}}{4 \pi} i k\right)^{3} \\
& +2 \frac{C_{2}^{(R)}}{C_{0}^{(R)}} k^{2}\left[1-2\left(\frac{m C_{0}^{(R)}}{4 \pi} i k\right)\right]+2 \frac{C_{2}^{\prime(R)}}{C_{0}^{(R)}} k^{2} \hat{p}^{\prime} \cdot \hat{p} \\
& \left.+\left(\frac{m C_{0}^{(R)}}{4 \pi} i k\right) \frac{k^{2}}{2 m^{2}}+\ldots\right\} .
\end{aligned}
$$

Here dependence on the cut-off was eliminated by defining renormalized parameters $C_{0}^{(R)}$, $C_{2}^{(R)}$, and $C_{2}^{\prime(R)}$ from the bare parameters $C_{0}(\Lambda), C_{2}(\Lambda)$, and $C_{2}^{\prime}(\Lambda)$ :

$$
\begin{gathered}
\frac{1}{C_{0}^{(R)}}=\frac{1}{C_{0}}+\frac{m}{2 \pi^{2}}\left[L_{1}+\left(\frac{2 C_{2}}{C_{0}}+\frac{1}{4 m^{2}}\right) L_{3}\right]+\ldots, \\
\frac{C_{2}^{(R)}}{\left(C_{0}^{(R)}\right)^{2}} \equiv \frac{C_{2}}{C_{0}^{2}}-\frac{m}{4 \pi^{2}}\left(\frac{R(0)}{\Lambda}+\frac{L_{1}}{2 m^{2}}\right)+\ldots, \quad C_{2}^{\prime(R)} \equiv C_{2}^{\prime}+\ldots
\end{gathered}
$$

Other terms coming from $R\left(k^{2} / \Lambda^{2}\right)$ are $\propto k^{4}$ or higher powers, and cannot be separated from higher-order contact interactions that I have not written down explicitly.

It is important to realize that Eq. (60) consists of two different expansions: a loop expansion and an expansion in the number of insertions of derivatives at the vertices or particle lines. The derivative expansion depends on the relative size of the coefficients $C_{2 n}^{(R)}$, for example $C_{2}^{(R)} Q^{2} / C_{0}^{(R)}$. The loop expansion is governed by $m C_{0}^{(R)} Q / 4 \pi$. The sizes of terms in both expansions thus depend on the absolute size of the $C_{2 n}^{(R)}$ 's. If one makes the simplest naturalness assumption that there is only a single mass scale $M$ and no fine-tuning in the underlying theory, we expect all parameters to scale with $M$ according to their mass dimension. It is convenient, however, to factor in a $4 \pi / m$ and write $C_{2 n}^{(\ldots)(R)} \sim 4 \pi / m M^{2 n+1}$. In this case, the derivative expansion is in $\sim(Q / M)^{2}$, while the loop expansion is in $\sim Q / M$ : the $T$-matrix in Eq. (60) is a simple expansion in powers of $Q / M$. However, in nuclear or molecular physics we are interested in shallow $S$-wave bound states 83 . A shallow (real or virtual) bound state means that a parameter $\alpha$ of the underlying theory is close to its critical value $\alpha_{c}$ for which a bound state is exactly at threshold. That is, the underlying theory is so fine-tuned that it has two distinct scales: $M$ and a lighter scale $\aleph=\left|\alpha / \alpha_{c}-1\right| M \ll M$

\footnotetext{
$\S \S$ The remaining case - that of an unnaturally small scattering length - has also been considered in the literature [56].
} 


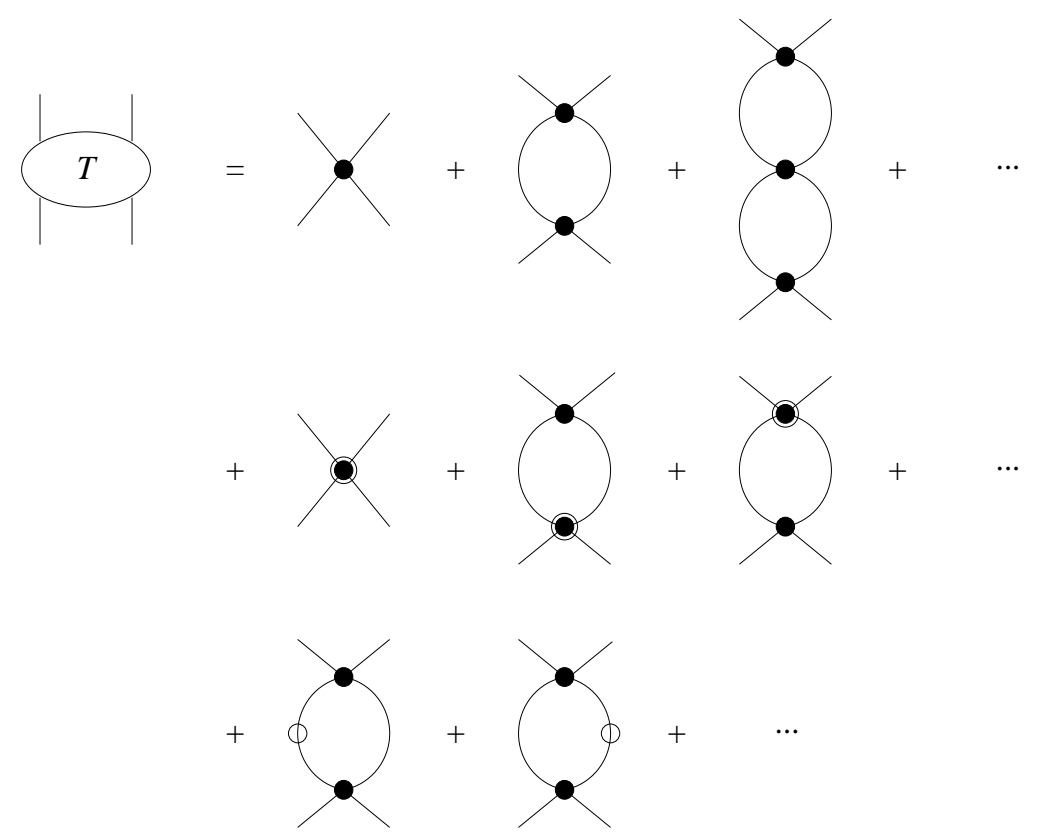

FIG. 2. First few terms of the two-particle amplitude $T$ in a natural EFT. Two solid lines represent a Schrödinger propagator; a circle on a line represents a $Q^{4}$ relativistic correction; a heavy dot stands for a $Q^{0}$ contact interaction, and a dot within a circle for a $Q^{2}$ contact interaction.

induced by the fine-tuning. I will deal with an $S$-wave shallow bound state by considering the class of theories where

$$
C_{2 n}^{(R)}=\frac{4 \pi}{m \aleph(M \aleph)^{n}} \gamma_{2 n}, \quad C_{2 n}^{\prime \ldots(R)}=\frac{4 \pi}{m M^{2 n+1}} \gamma_{2 n}^{\prime \ldots}
$$

with the $\gamma_{2 n}^{(\ldots)}$ 's dimensionless parameters of $O(1)$. The natural scenario is recovered when $\alpha$ is tuned out of $\alpha_{c}$, and $\aleph$ becomes comparable to $M$. The loop and derivative expansions contained in Eq. (60) are in $\sim Q / \aleph$ and $\sim Q^{2} / \aleph M$.

For $Q \ll \aleph$, the theory is perturbative in $Q / \aleph$. As $Q$ becomes comparable with , all the terms in the loop expansion are of the same order $(O(4 \pi / m \aleph))$ while corrections from derivatives go in relative powers of $\aleph / M \ll 1$ and can be accounted for systematically, at each order in $\aleph / M$. The amplitude is (see Fig. 3)

$$
\begin{aligned}
T_{o s}\left(k, \hat{p}^{\prime} \cdot \hat{p}\right) & =T_{o s}^{(0)}(k)+T_{o s}^{(1)}(k)+T_{o s}^{(2)}(k)+\ldots+T_{o s}^{(3 p)}\left(k, \hat{p}^{\prime} \cdot \hat{p}\right)+\ldots \\
& =\left(T_{o s}^{(0)}(k)\right)_{0}-2 C_{2}^{(R)} k^{2} \hat{p}^{\prime} \cdot \hat{p}+O\left((4 \pi / m \aleph)(\aleph / M)^{4}\right) .
\end{aligned}
$$

The $S$-wave component can be rewritten, up to higher order terms, as

$$
\begin{gathered}
\left(T_{\text {os }}(k)\right)_{0}=\left[\left(T_{\text {os }}^{(0)}(k)\right)^{-1}+T_{\text {os }}^{(1)}(k)\left(T_{\text {os }}^{(0)}(k)\right)^{-2}+T_{\text {os }}^{(2)}(k)\left(T_{o s}^{(0)}(k)\right)^{-2}+\ldots\right]^{-1} \\
=-\left[\frac{1}{C_{0}^{(R)}}-2 \frac{C_{2}^{(R)}}{\left(C_{0}^{(R)}\right)^{2}} k^{2}+4\left(\frac{\left(C_{2}^{(R)}\right)^{2}}{\left(C_{0}^{(R)}\right)^{3}}-\frac{C_{4}^{(R)}}{\left(C_{0}^{(R)}\right)^{2}}\right) k^{4}\right. \\
\left.+\frac{i m k}{4 \pi}\left(1+\frac{k^{2}}{2 m^{2}}\right)\right]^{-1}\left(1+O\left(\aleph^{4} / M^{4}\right)\right),
\end{gathered}
$$




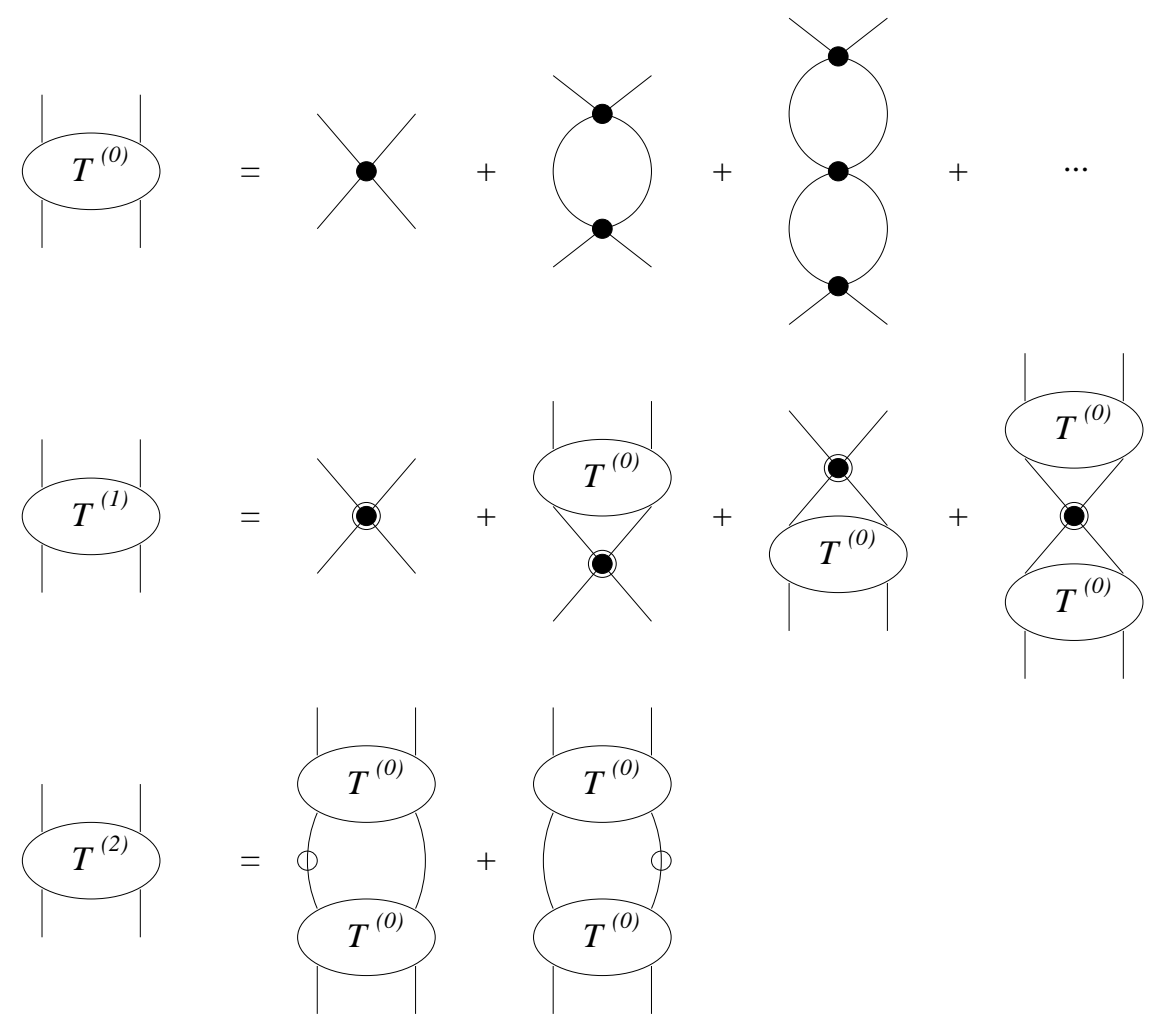

FIG. 3. First three orders of the two-particle amplitude $T$ in an EFT with a shallow bound state. Symbols as in Fig. 2.

where $C_{4}$ represents a certain combination of $Q^{4}$ contact interactions. By resumming the largest terms, we have produced a new expansion where the leading amplitude is $O(4 \pi / m(\aleph+$ $Q)$ ) and corrections go as $Q^{2} / M(\aleph+Q)$ or similar combinations. The amplitude (64) is nominally correct including $O\left((4 \pi / m \aleph)\left(\aleph^{2} / M^{2}\right)\right)$ only, but as we will see in a moment it is actually correct including $O\left((4 \pi / m \aleph)\left(\aleph^{3} / M^{3}\right)\right)$. As we consider larger $Q, Q \gtrsim \aleph$, the leading term becomes $O(4 \pi / m Q)$ and corrections get relatively more important, growing in powers of $Q / M$. The new expansion fails at momenta $\sim M$, as expected.

The amplitude (63) and (64) is in the form of an effective range expansion in each partial wave [57,58],

$$
(T(k))_{l}=\frac{4 \pi}{m k}\left(1+\frac{k^{2}}{2 m^{2}}+O\left(\frac{k^{4}}{m^{4}}\right)\right)^{-1}\left(\cot \delta_{l}-i\right)^{-1}
$$

with

$$
k^{2 l+1} \cot \delta_{l}=-\frac{1}{a_{2}^{(l)}}+\frac{r_{2}^{(l)}}{2} k^{2}+\sum_{n=2}^{\infty} v_{2}^{(n)(l)} k^{2 n}
$$

In the $S$ wave we obtain for the scattering length and the effective range

$$
a_{2}^{(0)}=\frac{m C_{0}^{(R)}}{4 \pi}, \quad r_{2}^{(0)}=\frac{16 \pi}{m C_{0}^{(R)}}\left(\frac{C_{2}^{(R)}}{C_{0}^{(R)}}+\frac{1}{4 m^{2}}\right) .
$$


In the $P$ wave, we find a scattering volume

$$
a_{2}^{(1)}=\frac{m C_{2}^{\prime(R)}}{6 \pi} .
$$

Higher moments $v_{2}^{(n)(l)}$ can be obtained from the $k^{2 n}$ terms that arise in higher order in the $Q / M$ expansion of the amplitude. For example, the $S$-wave shape parameter is

$$
v_{2}^{(2)(0)}=-\frac{16 \pi}{m C_{0}^{(R)}}\left(\left(\frac{C_{2}^{(R)}}{C_{0}^{(R)}}\right)^{2}-\frac{C_{4}^{(R)}}{C_{0}^{(R)}}\right) .
$$

An $S$-wave bound state can arise as a pole of the amplitude (64) at $k=i \kappa$,

$$
\kappa=\frac{4 \pi}{m C_{0}^{(R)}}\left(1+2 \frac{C_{2}^{(R)}}{C_{0}^{(R)}}\left(\frac{4 \pi}{m C_{0}^{(R)}}\right)^{2}+O\left((\aleph / M)^{2}\right)\right) .
$$

$C_{0}^{(R)}>0(<0)$ implies $\kappa>0(<0)$ and represents a real (virtual) bound state. (The residue of $i$ times the $S$-matrix at this pole is indeed positive (negative) if $\kappa>0(<0)$.) The binding energy $B_{2}$ to this accuracy is, of course, just the usual effective range relation among $B_{2}, a_{2}$ and $r_{2}$.

We see that an $l(>0)$-wave effective range parameter of mass dimension $\delta$ is $O\left(1 /(2 l+1) M^{\delta}\right)$. The $S$ wave is trickier. The scattering length $a_{2}^{(0)}$ scales with $\aleph$ rather than $M, a_{2}^{(0)}=O\left(\aleph^{-1}\right)$. The $S$-wave bound state is within the range of validity of the EFT, as $\kappa=O(\aleph)$ and the binding energy $B_{2}=\kappa^{2} / m+O\left(\aleph^{4} / M^{3}\right) \ll M$. It is clear that the bound state can be treated in a systematic expansion in $\aleph / M$. The two terms in the effective range $r_{2}^{(0)}$ (67) come at different orders. The main contribution originates in the contact interactions and scales with $M$, so that $r_{2}^{(0)}=O\left(M^{-1}\right)$. The relativistic correction, on the other hand, is smaller by $\sim M \aleph / m^{2} \sim \aleph / M$; this justifies the neglect of relativistic corrections in many situations and the usefulness of a non-relativistic framework in shallowbound-state problems. An underlying theory consisting of a non-relativistic potential can thus serve as a test for the scalings of $a_{2}^{(0)}$ and $r_{2}^{(0)}$. Indeed, it has been explicitly shown [59] that if the underlying theory consists of a non-relativistic potential of strength $\alpha$ and range $1 / M$, then near critical binding effective range parameters behave precisely in way derived above. As for the shape parameter, it appears from Eq. (69) that $v_{2}^{(2)(0)} \sim 1 / \aleph M^{2}$, but such a scaling is seen neither in phenomenological analyses nor in models. This implies that $\gamma_{4}=\gamma_{2}^{2} / \gamma_{0}+O(\aleph / M)$ : although each term in Eq. (69) comes at relative $O\left(Q^{4} / M^{2} \aleph^{2}\right)$, they are correlated and their sum is only of relative $O\left(Q^{4} / M^{3} \aleph\right)$. We conclude that the shape parameter appears at the same order as the first $P$-wave contribution when $Q \gtrsim \aleph$. Eq. (64) is therefore good up to $O\left(\aleph^{3} / M^{3}\right)$, as advertised. This means that the first three orders of the expansion of the amplitude - depicted in Fig. 3 - are pure $S$ wave and given solely by $C_{0}^{(R)}$ and $C_{2}^{(R)}$. It is easy to verify that, in order to avoid $M / \aleph$ enhancements in higher effective range parameters, there must be such correlations in higher order coefficients as well:

$$
C_{2 n}^{(R)}=C_{0}^{(R)}\left(C_{2}^{(R)} / C_{0}^{(R)}\right)^{n}+\text { smaller terms. }
$$




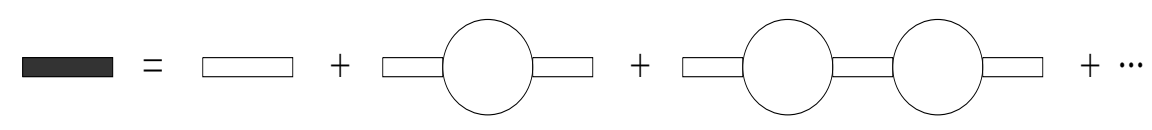

FIG. 4. The dressed dimeron propagator: a bar is the bare dimeron propagator, while the bubbles represent two-particle propagation.

We conclude that in the case of short-range interactions, the EFT approach, when applied consistently, is completely equivalent to the effective range expansion. The power counting described here and this equivalence were spelled out in a manifest scheme-independent way in Ref. [56], and in specific subtraction schemes in Refs. [60,61].

Dimeron. Although not necessary, it is possible to reorganize the EFT in order to account explicitly for the dominant correlations among the $C_{2 n}^{(R)}$ 's. Because they form a geometric series in $C_{2}^{(R)} / C_{0}^{(R)}$, we can sum the dominant correlation terms as we have done above with the $C_{0}^{(R)}$ contributions themselves. This procedure generates a four- $\psi$ interaction of the type $C_{0}^{(R)} /\left(1-2 C_{2}^{(R)} k^{2} / C_{0}^{(R)}\right)$; the first two terms in Eq. 64) follow immediately. Now, this new resummation resembles the exchange of an $s$-channel particle. This justifies the suggestion 62 that one can use a new EFT which involves, besides particles $\psi$, also a "dimeron" field $T$ with the quantum numbers of the shallow (real or virtual) two-body bound state. The leading terms in the most general Lagrangian consistent with the same symmetries as before are

$$
\mathcal{L}=\sigma T^{\dagger}\left(i \partial_{0}+\frac{1}{4 m} \vec{\nabla}^{2}-\Delta+\ldots\right) T-\frac{g}{\sqrt{2}}\left(T^{\dagger} \psi \psi+\text { h.c. }\right)+\ldots
$$

Here $g$ and $\Delta$ are parameters and $\sigma$ is the sign of the kinetic term. The Lagrangian (72) is equivalent to (57) if

$$
\sigma g^{2}=-\frac{C_{0}^{2}}{2 m C_{2}}, \quad \Delta=\frac{C_{0}}{2 m C_{2}} .
$$

Thus after renormalization $\left(g^{(R)}\right)^{2} / 2 \pi=O\left(M / m^{2}\right), \Delta^{(R)}=O(M \aleph / 2 m)$. “..." in Eq. (72) stand for terms with more derivatives, which are suppressed by powers of $\aleph / M$. Effects of non-derivative four- $\psi$ contact term can be absorbed in $g^{2} / \Delta$ and terms with more derivatives.

The coupling to two-particle states dresses the dimeron propagator. The dressed propagator contains bubbles as in Fig. 4, plus insertions of relativistic corrections. This amounts to a self-energy contribution proportional to the bubble integral. As we know, this integral is ultraviolet divergent and requires renormalization of the parameters of the Lagrangian (72). Relativistic corrections can be accounted for as in the EFT without the dimeron. The dimeron propagator in its rest frame $\left(p^{0} \equiv k^{2} / m-k^{4} / 4 m^{3}+\ldots\right)$ has the form

$$
S\left(p^{0}, \overrightarrow{0}\right)=\sigma \frac{\left(g^{(R)}\right)^{2}}{g^{2}} \frac{i}{\frac{k^{2}}{m}-\Delta^{(R)}+\frac{\sigma m\left(g^{(R)}\right)^{2}}{4 \pi} i k\left(1+\frac{k^{2}}{2 m^{2}}\right)+\ldots+i \epsilon} .
$$

The amplitude in this EFT is obtained directly from Eq. (74) - see Fig. 5. The result is identical to Eq. (64) to a given order. This is a more direct way to re-derive the effective range expansion, including the first few orders at once. The non-relativistic dimeron includes 


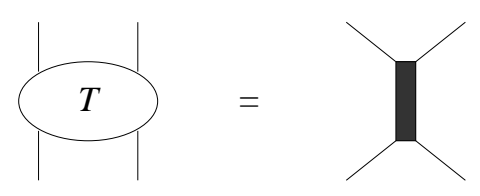

FIG. 5. The two-particle amplitude $T$ in the EFT with a dimeron field.

the first two orders in $\aleph / M$ correctly: in leading order only the mass term in the dimeron kinetic terms needs to be retained, and observables depend on the combination $\left(g^{(R)}\right)^{2} / \Delta^{(R)}$; in subleading order $g^{(R) 2}$ and $\Delta^{(R)}$ appear separately. The addition of relativistic corrections regains the first three orders. Corrections stemming from higher-derivative operators can be accounted for perturbatively, and will contribute to the $\psi \psi$ amplitude starting at relative $O\left((\aleph / M)^{3}\right)$. The drawback is a mixing of different orders in the $\aleph / M$ expansion, with a number of smaller, higher-order terms included.

The same real or virtual bound state as before exists near zero energy. The field $T$ corresponds to a light (ghost or normal) state of mass $\Delta^{(R)} \sim \aleph$ relative to $2 m$. It serves to introduce through its bare propagator two bare poles at the $k= \pm \sqrt{m \Delta}$. This is an undesired pole structure, but fortunately it changes character as the amplitude gets dressed, since the poles move to the imaginary axis. One ends up at the position of the shallow bound state, $k=i \kappa_{s}, \kappa_{s}=\kappa$ given by Eq. (70), while the other pole signals the breakdown of the EFT at $\kappa_{d}=O(M)$. The sign of the residue of $-i S$ at each of the poles shows that the shallow pole corresponds to a regular (composite) particle, while the deep pole corresponds to a ghost. The field $T$ can be used to describe the shallow bound state in processes other than $\psi \psi$ scattering, if one keeps in mind that the residue of $-i S$ at the pole is not 1 , but $\left(\left(g^{(R)}\right)^{2} / g^{2}\right)\left(\sigma /\left(1+\sigma m^{2} g^{2} / 8 \pi \sqrt{m B_{2}}\right)\right)$. Despite the resemblance, this is not an EFT with an "elementary" field for the bound state. At $Q \ll \aleph$, the EFT (with or without dimeron) can be matched onto a lower-energy EFT containing a heavy field for the bound state which does not couple to $\psi \psi$ states, but the dimeron field shows structure at $Q \gtrsim \aleph$, which arises from $\psi$ loops.

Schrödinger equation. Recall that an ordinary potential does not generate an $1 / r$ behavior of the wave-function close to the origin because action of the Laplacian on such a singularity produces a delta function: $\nabla^{2}(1 / r)=-4 \pi \delta(\vec{r})$. A local delta-function potential in turn is too singular to admit a solution of this type because then the potential side of the Schrödinger equation becomes $V \Psi \sim \delta(\vec{r}) / r$, more singular than the kinetic side. This is the translation into Schrödinger language of the need for regularization and renormalization of the bubble diagrams.

The effects of renormalization on the on-shell amplitude are two-fold. One is to replace the potential (58) by

$$
V^{(R)}(k)=C_{0}^{(R)}+2 C_{2}^{(R)} k^{2}+\ldots
$$

The other is to substitute the (free) Schrödinger propagator (43) by

$$
G_{0}^{(R)}(r ; k)=-\frac{i m k}{4 \pi}(1+O(k r))
$$


It can be shown [56] that this is equivalent to the usual Schrödinger propagation with an "effective renormalized potential" or pseudopotential. The renormalized Schrödinger equation is

$$
-\frac{1}{m}\left(\nabla^{2}+k^{2}\right) \Psi(\vec{r})=-\left(C_{0}^{(R)}+2 C_{2}^{(R)} k^{2}+\ldots\right) \delta(\vec{r}) \frac{\partial}{\partial r}(r \Psi(\vec{r})) .
$$

Recalling the connections between EFT and effective range parameters, we see that this is nothing but a generalization of the well-known pseudopotential of Fermi and Breit 63 65. Therefore, renormalization of an EFT with only short-range interactions leads to a Schrödinger equation with a pseudopotential determined by renormalized parameters, rather than a Schrödinger equation with a local, smeared delta-function-type potential determined by bare parameters.

Further insight into the effect of renormalization can be gained by looking at the solution of Eq. (77). The effect of the $\frac{\partial}{\partial r} r$ operator in the pseudopotential is to soften the singularity at the origin, replacing $\delta(\vec{r}) / r$ by $\delta(\vec{r})$. Indeed, we can find the solution of Eq. (77) by expanding $\Psi(\vec{r})=\sum_{n=-\infty}^{+\infty} A_{n} r^{n}$ at small $r$. The result is completely determined by $A_{-1}$. Now, the same solution follows from

$$
-\frac{1}{m}\left(\nabla^{2}+k^{2}\right) \Psi(\vec{r})=\frac{4 \pi}{m} r \delta(\vec{r}) \Psi(\vec{r})
$$

— which for any $\vec{r} \neq 0$ is the free Schrödinger equation — with a peculiar boundary condition at the origin,

$$
\left.\frac{\partial}{\partial r} \ln (r \Psi(\vec{r}))\right|_{r=0}=-\frac{4 \pi}{m C_{0}^{(R)}}\left(1-2 \frac{C_{2}^{(R)}}{\left(C_{0}^{(R)}\right)^{2}} k^{2}+\ldots\right)
$$

We recognize the first term in Eq. (79) as the boundary condition of Bethe and Peierls, and Breit [1,64]. The renormalized EFT of only short-range interactions is equivalent to an energy expansion of the most general condition on the logarithmic derivative of the wavefunction at the origin. The physics behind these Schrödinger formulations is clear if we look at the resulting radial wave-functions $r \psi(r)=u(r)$, which obey a free radial equation. (i) For $k^{2}=-\kappa^{2}<0$,

$$
u(r)=c_{(-)} e^{-\kappa r}
$$

with $c_{(-)}$a normalization factor and $\kappa$ given by Eq. (70). (ii) For $k^{2} \geq 0$,

$$
u(r)=c_{(+)}\left(\sin k r-\frac{a_{2}^{(0)} k}{1-a_{2}^{(0)} r_{2}^{(0)} k^{2} / 2+\ldots} \cos k r\right),
$$

with $c_{(+)}$a normalization factor and $a_{2}^{(0)}$ and $r_{2}^{(0)}$ given in Eqs. (67). By changing the unspecified dependence of $c_{(+)}$on $k$ and $\kappa_{s, d}$ we do not change the underlying pole structure of the $\psi \psi$ amplitude, but we do change the Jost functions. This is a reflection of the fact that the EFT cannot distinguish among short-range potentials which produce the same asymptotic behavior. Indeed, it is not difficult to see that with appropriate choices of $c_{(+)}$ 
we can cover all situations considered in Ref. [66]. The EFT shrinks to the origin the complicated wiggles that the "underlying" wave-function might have at small distances, thus replacing them with a smooth behavior that matches onto the tail of the underlying wave-function. The effective wave-function is all tail for the tail is all one can see from far away.

Potential and regularization. I have so far avoided using specific regularization schemes in order to emphasize features of the renormalized theory, which is all that determines observables anyway. Now a few words are spent on the related issues of the role of potentials and of regularization schemes. Some of these remarks have been presented in Ref. [56], and shown to be supported by a careful numerical investigation [67].

It is clear that iteration of the potential is superfluous in any partial wave without finetuning: within its region of validity, the EFT is perturbative, and any bound states, if they exist, are beyond the range of the EFT. Iteration of the potential includes only part of the higher-order terms, and this incomplete set cannot a priori be taken seriously. It has been shown explicitly that the iteration of momentum-independent contact interactions does not reproduce the non-analytical behavior of a toy natural underlying theory [68]. On the other hand, since the error induced by iteration is small, it does not affect observables much. The more interesting cases are the ones with fine-tuning in the underlying theory.

The appropriate formula for the amplitude, Eq. (64), was obtained by iterating the $Q^{0}$ part of the potential to all orders and by using perturbation theory for the corrections. The same result can be obtained more easily by first summing the bubbles with the full $V\left(p^{\prime}, p\right)$ and then expanding in powers of the energy. For example, keeping track only of the $Q^{2}$ terms in the $S$-wave,

$$
\left(T_{o s}(k)\right)_{0}=-\left(\frac{1}{C_{0}+2 C_{2} k^{2}}-I(k)\right)^{-1}, I(k)=\left(1+\frac{k^{2}}{2 m^{2}}\right) I_{0}(k)-\left(2 \frac{C_{2}}{C_{0}}+\frac{1}{4 m^{2}}\right) \frac{m L_{3}}{2 \pi^{2}}
$$

This corresponds to the standard procedure of iterating the full potential, but it is now obvious that to remove the infinities we have to expand

$$
\frac{1}{C_{0}+2 C_{2} k^{2}}=\frac{1}{C_{0}}-2 \frac{C_{2}}{C_{0}^{2}} k^{2}+\ldots,
$$

and consistently neglect $Q^{4}$ terms. We are then back to Eq. (64) truncated at $O(\aleph / M)$. Terms of $O\left(Q^{4}\right)$ and higher can only be made regulator-independent by renormalizing higherorder parameters in the Lagrangian. Indeed, as we have seen before, $O\left(Q^{4}\right)$ terms in $V\left(p, p^{\prime}\right)$ will contribute $k^{4}$ terms to Eq. (82).

Regulator-dependence is allowed insofar as it is small, that is, of the same magnitude as smaller terms that have been neglected. Cutoff dependence may arise from two sources. Some cutoff dependence comes from $R\left(k^{2} / \Lambda^{2}\right)$, for it contains arbitrarily high powers of $Q / \Lambda$. Further cutoff dependence creeps in through bare parameters if we iterate the full potential to all orders but neglect to make the expansion (83). For example, to $O\left((\aleph / M)^{2}\right)$, by solving the Schrödinger equation with a finite cut-off we induce a shape parameter

$$
v_{2}^{(2)(0)}=-\frac{16 \pi C_{2}^{2}}{m C_{0}^{3}}-\frac{2}{\pi \Lambda^{3}}\left(R^{\prime}(0)+\frac{\Lambda^{2}}{2 m^{2}} R(0)\right)-\frac{r_{2}^{(0)}}{8 m^{2}} .
$$


We see that apart from a regularization-independent relativistic correction, cutoff dependence enters through both $R$ and the bare parameter ratio $C_{2}^{2} / C_{0}^{3}$. It is natural to ask what are the constraints on regularization procedures imposed by the requirement that both sources of cutoff dependence are small, as in more complicated situations it might be difficult to carry the step (83) explicitly. Note that the EFT theory with a dimeron field automatically performs the step (83), and therefore reduces differences among regularization schemes to the $R$ they generate.

In a cutoff regularization, the form of $F\left(l^{2} / \Lambda^{2}\right)$ is chosen explicitly. The error induced by $R$ is removed order-by-order, the relative residual error being $O(\aleph / \Lambda)$. Finite cut-offs have been strongly advocated in Refs. [25,70]. Keeping a cutoff $\Lambda \sim M$ has one useful consequence. In this case, the large renormalized parameters $C_{2 n}^{(R)}$ can be generated by natural-size bare coefficients $C_{2 n}=O\left(4 \pi / m M^{2 n+1}\right)$. For example,

$$
C_{0}(\Lambda)=-\frac{2 \pi^{2}}{\theta_{1} m \Lambda}\left(1+\pi \aleph / 2 \gamma_{0} \theta_{1} \Lambda+\ldots\right)
$$

is of natural size even though $C_{0}^{(R)}=4 \pi \gamma_{0} / m \aleph$ is large. What a cutoff does is to give a scale for the bare parameters to be fine-tuned against. Before renormalization each loop contributes a large factor $\propto \Lambda$ but each $C_{0}$ compensates with a small factor $\propto \Lambda^{-1}$, resulting a net $\sim\left(1+\pi(\aleph+Q) / 2 \theta_{1} \Lambda+\ldots\right)$. The non-perturbative character of the theory is evident: it generates cancellations among the leading terms, and the relevant physics is contained in the terms that are suppressed by $\aleph / \Lambda$ or $Q / \aleph$. These remarks can be couched in a renormalization group analysis for the potential [69].

In this way the ratio of bare parameters can be made of natural size as well. The difference between a truncation of the rhs of Eq. (83) and the lhs is then no bigger than other neglected small effects such as higher-order interactions in the Lagrangian: for example, the shape parameter (84) induced by the cutoff is in this case $O\left(1 / M^{3}\right)$. A power expansion of the bare potential followed by its iteration to all orders is under these circumstances no worse than a perturbation treatment of the corrections to leading order. In this case the power counting (45) applies to the potential itself: since here the potential is a tree-level object involving four fermion fields

$$
\nu=d
$$

where $d$ is the number of derivatives at the vertex. Truncating the potential at certain order accounts correctly for the first few terms in the expansion of the amplitude but includes also a subset of higher-order terms. We can actually invert the previous reasoning and use the freedom introduced by a finite cutoff to save some work. Given a regularization procedure, we can further fine-tune $\Lambda$ to its "optimal" value that improves agreement with data. If we are dealing with a single channel, this is a consistent procedure: it simply means that $O\left(Q^{4}\right)$ contributions have been included, but they do not need to be written explicitly because the corresponding bare parameter is zero. In general, however, only part of the higher order effects can be accounted for in this manner. As $\Lambda \rightarrow \infty$ the size of implicitly kept higherorder terms decreases. For example, all terms in Eq. (84) — apart from the finite relativistic correction - then vanish. A consequence of this, noted in Ref. [71], is that the first two terms in $V\left(p, p^{\prime}\right)$ are renormalizable in such non-perturbative context. 
To any given order, equations relating bare and renormalized parameters will be truncated, and beyond leading order, be highly non-linear — see Eqs. (61), for example. Inverting them, we can find the bare parameters in terms of the renormalized parameters (known from data) and the cutoff: $C_{2 n}=C_{2 n}\left(C_{2 n}^{(R)} ; \Lambda\right)$. This of course means that the bare parameters are not observables, depending on the scheme chosen. Given $C_{2 n}^{(R)}$ 's, whether one finds real solutions $C_{2 n}$ for any $\Lambda$ depends on the actual values of the $\theta_{n}$ 's in Eq. (59). In particular, to $O\left((\aleph / M)^{2}\right)$ the important term for large $\Lambda$ is $C_{2}^{(R)} \theta_{3} \Lambda^{3}$; the sign of $C_{2}^{(R)}$ being that of $r_{2}^{(0)}$, it is the sign of $\theta_{3}$ which is of concern. It was pointed out in Ref. [72 that the most obvious cutoff schemes (such as a sharp momentum-space cutoff and a coordinate-space regularization by square wells) imply $\theta_{3}>0$. Imposing real bare parameters in such schemes, it is found that $r_{2}^{(0)} \leq 2 / \Lambda$ for large $\Lambda$, or in other words, that the limit $\Lambda \rightarrow \infty$ can only be taken if $r_{2}^{(0)} \leq 0$. This is a reformulation in field-theoretical language of Wigner's theorem [73]: $r_{2}^{(0)}$ cannot be positive if we take a sequence of ever shorter-range, (possibly non-local) hermitian potentials in a coordinate space Schrödinger equation. While such a constraint is unusual, it is doubtful that it is of much relevance to EFTs applied consistently to a certain order. First, this is obviously a regularization-scheme-dependent issue: it is easy to construct (smooth) cutoff schemes such that $\theta_{3} \leq 0$ [74]. In the worst case scenario, the above constraint is a result of attempting to remove the cutoff in an inadequate regularization scheme. Second, this constraint only arises from a constraint imposed on unobservable bare parameters. No matter what process one is considering in the low-momentum regime, the bare parameters never appear, except in the right combinations with the cutoff that produce renormalized parameters: an imaginary bare parameter is of no relevance. Indeed, the Schrödinger equation for the "renormalized potential" (77) violates the assumptions made in Ref. [72 about the nature of the potential. Finally, even if one insists on the unfortunate choice of regulator and the reality constraint on bare parameters, a positive $r_{2}^{(0)}$ can still be achieved by keeping the cutoff finite and of the order of the mass scale of the underlying theory, $\Lambda \sim M$.

An alternative regularization is dimensional regularization. $F$ is implicit when we extend the dimension of space to a sufficiently small $D-1$ such that the integral converges; the scale introduced is usually denoted $\mu$. With minimal subtraction, $\theta_{n}=0$ and $R(x)=0$. A nonminimal subtraction ("PDS") scheme was introduced in Ref. 60], in which a pole in $D=3$ was subtracted. This particular scheme corresponds to $\theta_{1}=\pi / 2, \theta_{n>1}=0$, and $R(x)=0$. For $\mu=0$ this scheme reduces to minimal subtraction. It is not difficult to relate parameters in subtraction and cutoff schemes. If we call the cutoff parameter $\Lambda=\bar{\Lambda}(\mu)+\pi \mu / 2 \theta_{1}$, we can write

$$
I_{2 n}(k)=-\frac{m}{2 \pi^{2}}\left[\bar{\Lambda} \sum_{i=0}^{n} k^{2 i} \varpi_{n-i}(\bar{\Lambda}, \mu)+\frac{\pi}{2} k^{2 n}(i k+\mu)+\frac{k^{2(n+1)}}{\bar{\Lambda}} \bar{R}\left(k^{2} / \bar{\Lambda}^{2}, \mu / \bar{\Lambda}\right)\right],
$$

where $\varpi_{n-i}(\bar{\Lambda}, \mu)$ and $\bar{R}\left(k^{2} / \bar{\Lambda}^{2}, \mu / \bar{\Lambda}\right)$ are related to the $\theta_{n}$ 's and $R\left(k^{2} / \Lambda^{2}\right)$. As we have seen, terms that grow with the cutoff can be cancelled by the counterterms $C_{2 n}(\Lambda)$. If one defines new parameters $C_{2 n}(\mu)$ as $\bar{\Lambda}$-independent functions of the $C_{2 n}(\Lambda)$ and the $\varpi_{n-i}(\bar{\Lambda}, \mu)$, we can express the renormalized quantities $C_{2 n}^{(R)}$ as $\mu$-independent functions of the $C_{2 n}(\mu)$ and $\mu$. For example, in leading order 


$$
\frac{1}{C_{0}(\mu)}=\frac{1}{C_{0}(\Lambda)}+\frac{m \theta_{1} \bar{\Lambda}}{2 \pi^{2}}
$$

For $\mu, k \ll \bar{\Lambda}$ contributions from $\bar{R}$ are small; in the limit $\bar{\Lambda} \rightarrow \infty$ (with $\mu$ fixed) they vanish and the theory with cutoff can be rewritten in terms of the $C_{2 n}(\mu)$ and

$$
I_{2 n}(k)=-\frac{m}{4 \pi} k^{2 n}(i k+\mu)
$$

which is equivalent to the theory with integrals subtracted at $k^{2}=-\mu^{2}$ [10,61]. Other subtraction schemes in dimensional regularization have been discussed in Ref. [75].

Dimensional regularization suffers from no errors generated by $R$. Minimal subtraction, first applied to this problem in Ref. [76], is special because it forces the bare parameters to coincide with the renormalized ones and be unnaturally large. Because no cutoff dependence is visible, if one works to $Q^{2}$ terms it is not immediately apparent that $1 /\left(C_{0}+2 C_{2} k^{2}\right)$ has to be expanded in $k^{2}$. It was one of the results of Ref. [76] that a fitting of the ${ }^{1} S_{0} N N$ phase shift based on the effective range expansion worked much better than one based on the form $1 /\left(C_{0}+2 C_{2} k^{2}\right)$. One can understand the failure of the $1 /\left(C_{0}+2 C_{2} k^{2}\right)$ fit by noticing that the induced spurious shape parameter (84) is here $v_{2}^{(2)(0)}=a_{2}^{(0)} r_{2}^{(0) 2} / 4-3 r_{2}^{(0)} / 8 m^{2}+1 / 4 a_{2}^{(0)} m^{4}$, which is $O\left(1 / \aleph M^{2}\right)$. Since there is no regulator to blame the fine-tuning on, the finetuning contaminates all other induced terms as well: they are all automatically large if $a_{2}^{(0)}$ is large. In dimensional regularization with minimal subtraction one cannot substitute the perturbative treatment of corrections by the iteration of the whole potential without reducing the range of applicability of the theory from $M$ to the ratio of parameters in the power expansion of the contact interactions, which is $\sqrt{\aleph M}$. The perturbative treatment of corrections can still be carried out with dimensional regularization, or equivalently one can use the EFT with a dimeron field. This is true of minimal subtraction or other subtraction schemes. Of those, PDS and other schemes with a small subtraction scale are particularly convenient. For example, from Eqs. (85) and (88) we see that natural-size bare coefficients translate into

$$
C_{0}(\mu)=\frac{4 \pi}{m\left(-\mu+\aleph / \gamma_{0}\right)}
$$

$C_{0}(\aleph)=O(4 \pi / m \aleph)$ is a large running coupling. One can show that other $C_{2 n}(\mu)$ are also of the same size as their renormalized counterparts $C_{2 n}^{(R)}$, and that they satisfy relations analogous to (71). We see that the power counting valid for the renormalized theory is manifest when the theory is formulated directly in terms of a small mass scale $\mu$ and running parameters $C_{2 n}(\mu)$ 77,60.6.

Spin and isospin. Generalization to include spin $S$ and isospin $I$ is straightforward. In $N N$ scattering, $S, I=0,1$. The Pauli principle requires $I+S+L$ be odd, where $L$ is the orbital angular momentum. Channels are labeled by ${ }^{2 S+1} L_{J}$, where $J$ is the total angular momentum. There can be mixing between $S=1$ channels with $L=J-1$ and $L=J+1$, generated by the tensor operator $S_{12}(q) \equiv 3 \vec{q} \cdot \vec{\sigma}_{1} \vec{q} \cdot \vec{\sigma}_{2} / q^{2}-\vec{\sigma}_{1} \cdot \vec{\sigma}_{2}$. The corresponding amplitude can be expressed in terms of a mixing angle $\varepsilon_{J}$,

$$
(T(k))_{J-1 ; J+1}=-i \frac{2 \pi}{m k}\left(\begin{array}{cc}
e^{2 i \delta_{J-1}} \cos 2 \varepsilon_{J}-1 & i e^{i\left(\delta_{J-1}+\delta_{J+1}\right)} \sin 2 \varepsilon_{J} \\
i e^{i\left(\delta_{J-1}+\delta_{J+1}\right)} \sin 2 \varepsilon_{J} & e^{2 i \delta_{J+1}} \cos 2 \varepsilon_{J}-1
\end{array}\right) .
$$


One can use Fierz reordering to reduce the number of independent contact interactions to two $Q^{0}$ terms [10], seven $Q^{2}$ terms [12], and so on: in terms of $\vec{q} \equiv \vec{p}-\vec{p}^{\prime}$ and $\vec{k} \equiv \frac{1}{2}\left(\vec{p}+\vec{p}^{\prime}\right)$, one possible choice is 15,16]

$$
\begin{aligned}
V\left(p, p^{\prime}\right)= & C_{0}^{(S)}+C_{0}^{(T)} \vec{\sigma}_{1} \cdot \vec{\sigma}_{2}+C_{2}^{(1)} \vec{q}^{2}+C_{2}^{(2)} \vec{k}^{2}+\left(C_{2}^{(3)} \vec{q}^{2}+C_{2}^{(4)} \vec{k}^{2}\right) \vec{\sigma}_{1} \cdot \vec{\sigma}_{2} \\
& +i C_{2}^{(5)} \frac{\vec{\sigma}_{1}+\vec{\sigma}_{2}}{2} \cdot(\vec{q} \times \vec{k})+C_{2}^{(6)} \vec{q} \cdot \vec{\sigma}_{1} \vec{q} \cdot \vec{\sigma}_{2}+C_{2}^{(7)} \vec{k} \cdot \vec{\sigma}_{1} \vec{k} \cdot \vec{\sigma}_{2}+\ldots
\end{aligned}
$$

The two $Q^{0}$ terms and two of the $Q^{2}$ terms contribute to the two $S$-wave channels, ${ }^{3} S_{1}$ and ${ }^{1} S_{0}$. The remaining five $Q^{2}$ terms contribute to ${ }^{3} S_{1}-{ }^{3} D_{1}$ mixing and to the four $P$-wave channels, ${ }^{1} P_{1},{ }^{3} P_{0},{ }^{3} P_{1}$, and ${ }^{3} P_{2}$. At higher powers of momenta, contributions to $D$ and other waves appear.

Since there are shallow bound states in both $S$-wave channels, QCD must belong to the universality class of EFTs that obey the scaling (62) with $M=M_{n u c}$. The only complication arises from the mixing generated by the $C_{2}^{(6)}$ term. Analogously to the effective range expansion, we can expand

$$
\frac{1}{2} \sin 2 \varepsilon_{1}=\frac{k^{3} a_{2}^{(0)}}{\sqrt{1+k^{2} a_{2}^{(0) 2}}} \sum_{n=0}^{\infty} u_{2}^{(n)(1)}\left(k a_{2}^{(0)}\right) k^{n},
$$

where the $u_{2}^{(n)(1)}\left(k a_{2}^{(0)}\right)$ 's are certain functions of the combination $k a_{2}^{(0)}$ (but not of $k$ or $a_{2}^{(0)}$ separately). Models suggest that $u_{2}^{(n)(1)}\left(k a_{2}^{(0)}\right)=O\left(1 / M_{n u c}^{(2+n)}\right)$. This expansion is reproduced by the EFT provided

$$
C_{2}^{(6)(R)}=\frac{4 \pi}{m \aleph M^{2}} \gamma_{2}^{(6)}
$$

with $\gamma_{2}^{(6)}$ dimensionless of $O(1)$. For example, $u_{2}^{(0)(1)} \propto C_{2}^{(6)} /\left(C_{0}^{(S)}+C_{0}^{(T)}\right) \sim 1 / M_{n u c}^{2}$. Such an argument can be generalized to higher orders. A local operator that connects a state with angular momentum $l$ to another with angular momentum $l^{\prime}$ involves at least $l+l^{\prime}$ derivatives and first appears at $O\left(\left(Q / M_{n u c}\right)^{l+l^{\prime}}\right)$ if either $l$ or $l^{\prime}$ is zero, or at $O\left(\left(Q / M_{n u c}\right)^{l+l^{\prime}+1}\right)$ if both $l$ and $l^{\prime}$ are non-zero, relative to the lowest $\left(O(4 \pi / m \aleph)\right.$ ) contribution (from $\left.l=l^{\prime}=0\right)$.

Power counting then indicates that the low-energy phase shifts should be larger in the $S$ waves, with the $Q^{0}$ terms being treated non-perturbatively. The other waves, $P, D, F$, and so on, should be amenable to perturbation theory up to ever increasing energy, as we have seen that the suppression factor is $\sim 1 /(2 l+1) M_{n u c}$ in an $l$ wave. We also expect the waves to be roughly in decreasing order of magnitude as orbital angular momentum increases, as they receive contributions at increasingly higher orders. These conclusions are qualitatively substantiated by a PSA [78].

Up to relative $O\left(Q / M_{n u c}\right)$ only $S$ waves are present. In each $S$-wave channel we have one non-derivative interaction at leading order and one two-derivative interaction at subleading order, which we can fit to the scattering length and to the effective range, respectively. From the singlet parameters $a_{p p}^{\left({ }^{1} S_{0}\right)}=-17.3 \mathrm{fm}, a_{n p}^{\left({ }^{1} S_{0}\right)}=-23.75 \mathrm{fm}, a_{n n}^{\left({ }^{1} S_{0}\right)}=-18.8 \mathrm{fm}$, $r_{p p}^{\left({ }^{1} S_{0}\right)}=2.85 \mathrm{fm}, r_{n p}^{\left({ }^{1} S_{0}\right)}=2.75 \mathrm{fm}$, and $r_{n n}^{\left({ }^{1} S_{0}\right)}=2.75 \mathrm{fm}$ [79 we find the averages

$$
C_{0}^{(R)\left({ }^{1} S_{0}\right)}=1.3 \cdot 10^{-3} \mathrm{MeV}^{-2}, \quad C_{2}^{(R)\left({ }^{1} S_{0}\right)}=4.5 \cdot 10^{-7} \mathrm{MeV}^{-4} .
$$




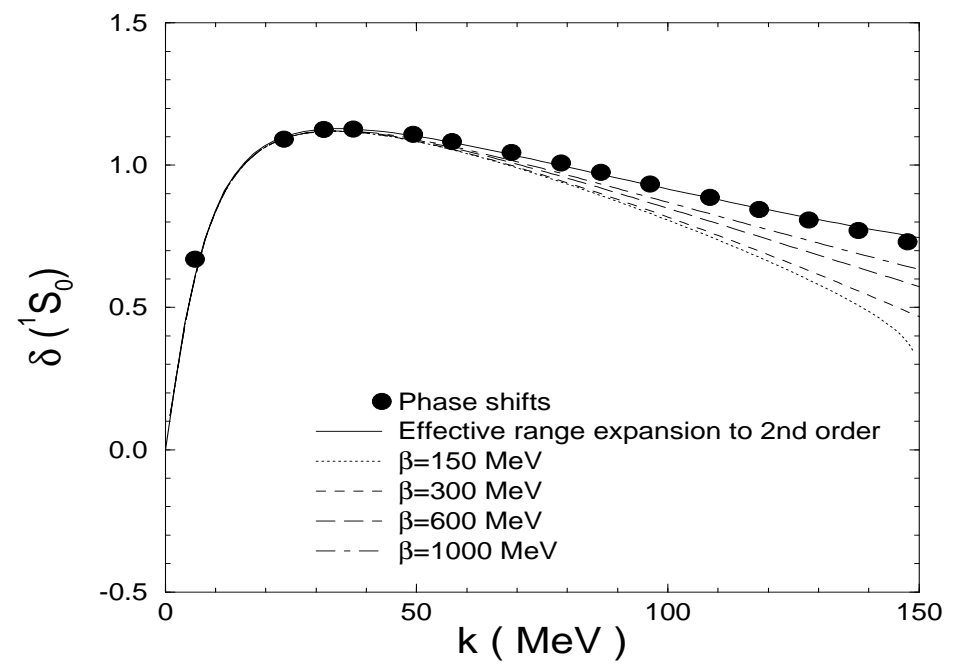

FIG. 6. ${ }^{1} S_{0} N N$ phase shift in radians as function of the center-of-mass momentum in the EFT to $O\left(Q / M_{n u c}\right)$ for several values of the cutoff $\beta$ (curves), compared to the Nijmegen phase shift analysis (circles). Figure courtesy of D. Phillips.

From the triplet parameters $a_{2}^{\left({ }^{3} S_{1}\right)}=5.42 \mathrm{fm}$ and $r_{2}^{\left({ }^{3} S_{1}\right)}=1.75 \mathrm{fm} 80$ we find

$$
C_{0}^{(R)\left({ }^{3} S_{1}\right)}=3.6 \cdot 10^{-4} \mathrm{MeV}^{-2}, \quad C_{2}^{(R)\left({ }^{3} S_{1}\right)}=2.1 \cdot 10^{-8} \mathrm{MeV}^{-4} .
$$

Taking a channel-average $\aleph=30 \mathrm{MeV}$ and $M_{n u c}=140 \mathrm{MeV}$, these translate into reasonable $\gamma_{0}^{\left({ }^{1} S_{0}\right)} \simeq 2.9, \gamma_{2}^{\left({ }^{1} S_{0}\right)} \simeq 4.2, \gamma_{0}^{\left({ }^{3} S_{1}\right)} \simeq 0.8$, and $\gamma_{2}^{\left({ }^{3} S_{1}\right)} \simeq 0.2$. One can then predict the phase shifts as functions of energy. As an example, in Fig. 6 we see the resulting ${ }^{1} S_{0}$ phase shift as function of $k$ for a number of cutoffs $\Lambda=\beta$ [81, compared to the values of the Nijmegen PSA [78. Cutoff dependence is significant only at momenta of $O\left(m_{\pi}\right)$ where this EFT should fail. The effective range expansion, which corresponds to the $\Lambda \rightarrow \infty$ limit, agrees well with the PSA up to such momenta. Similar conclusion holds for the ${ }^{3} S_{1}$ phase shifts. In the case of the ${ }^{3} S_{1}$ channel, we can alternatively use the deuteron binding energy to determine $C_{0}^{(R)\left({ }^{3} S_{1}\right)}$, the fitting difference being of higher order. This is a more natural strategy for studying processes that involve the deuteron.

At relative $O\left(\left(Q / M_{n u c}\right)^{2}\right)$, mixing between ${ }^{3} S_{1}$ and ${ }^{3} D_{1}$ states appears thanks to $C_{2}^{(6)(R)}$, and relativistic corrections need to be included in $S$ waves ***. $C_{2}^{(6)(R)}$ can be determined from the low-energy behavior of $\epsilon_{1}$ and then deuteron parameters can be predicted, or vice-versa. Using standard formulas in terms of integrals over wave-functions, deuteron properties found with $C_{2}^{(6)(R)}$ fitted to a deuteron asymptotic $D / S$ ratio $\eta=0.025$ (as in the Argonne V18 potential [82]) are given in Table [1] [83]. (Electromagnetic quantities refer to the contributions from lowest-order $\gamma N N$ couplings only, not to a consistent calculation which would include subleading one- and two-nucleon effects.)

*** The latter are numerically small, however, and can usually be neglected. 
TABLE II. Results from EFT fits to subleading order for various cut-offs $\Lambda$ and experimental values for the deuteron binding energy $(B)$, magnetic dipole moment $\left(\mu_{d}\right)$, electric quadrupole moment $\left(Q_{E}\right)$, asymptotic $D / S$ ratio $(\eta)$, and $D$-state probability $\left(P_{D}\right)$.

\begin{tabular}{ccccc}
\hline \hline Deuteron & \multicolumn{3}{c}{$\Lambda(\mathrm{MeV})$} & \\
\cline { 2 - 4 } quantities & 150 & 216 & 250 & Experiment \\
\hline$B(\mathrm{MeV})$ & 1.799 & 2.211 & 2.389 & $2.224579(9)$ \\
$\mu_{d}\left(\mu_{N}\right)$ & 0.868 & 0.846 & 0.828 & $0.857406(1)$ \\
$Q_{E}\left(\mathrm{fm}^{2}\right)$ & 0.231 & 0.288 & 0.305 & $0.2859(3)$ \\
$\eta$ & $0.025(\mathrm{fit})$ & $0.025(\mathrm{fit})$ & $0.025(\mathrm{fit})$ & $0.0271(4)$ \\
$P_{D}(\%)$ & 2.11 & 5.89 & 9.09 & \\
\hline \hline
\end{tabular}

The theory can be extended to higher orders in obvious fashion.

Electromagnetic corrections. Because of the non-relativistic nature of heavy particle propagation, electromagnetic interactions are dominated by Coulomb-photon exchange. One-Coulomb-photon exchange is $O\left(e^{2} / Q^{2}\right)$, while, using the power counting developed above, iterated once it is $O\left(m e^{4} / 4 \pi Q^{3}\right)$. Therefore, Coulomb-photon exchange is (non-) perturbative for $Q \gtrsim \alpha m(Q \lesssim \alpha m)$.

In the case of nucleons, $\alpha m_{N} \simeq 7 \mathrm{MeV}$, which almost coincides with the light scale in the $S$-wave channel where $p p$ scatter, $\aleph^{\left({ }^{1} S_{0}\right)} \sim 8 \mathrm{MeV}$; that is, numerically, $\alpha \sim \aleph^{\left({ }^{1} S_{0}\right)} / m_{N}$. For $Q \ll \aleph^{\left({ }^{1} S_{0}\right)}$, Coulomb interactions have to be iterated to all orders, while we can treat strong interactions generated by the potential (92) as perturbative insertions. Conversely, for $Q \gg \aleph^{\left({ }^{1} S_{0}\right)}$, we can add electromagnetic interactions as perturbations.

At $Q \sim \aleph^{\left({ }^{1} S_{0}\right)}$, both one-Coulomb-photon exchange and the momentum-independent contact interaction are of the same order and need to be iterated to all orders. Corrections can most simply be classified taking $\alpha \sim \aleph^{\left({ }^{1} S_{0}\right)} / M_{n u c}$, and then recognizing that an extra numerical suppression affects electromagnetic corrections because $M_{n u c} / m_{N} \ll 1$. At $\left.O\left(\aleph^{1}{ }^{1} S_{0}\right) / M_{n u c}\right)$, besides the two-derivative contact interaction, the most important electromagnetic correction appears due to vacuum polarization, as it comes suppressed by a single power of $\alpha$. (Actually, the more typical suppression of photon loops is $\alpha / \pi$, reducing further the impact of this effect.) At $O\left(\left(\aleph^{\left({ }^{1} S_{0}\right)} / M_{n u c}\right)^{2}\right)$, besides relativistic corrections to the $S$ wave, transverse-photon exchange generates magnetic interactions. More exotic electromagnetic interactions — such as two-photon exchange generated by the Thomson seagullappear in higher orders. This ordering of electromagnetic interactions [84] is in qualitative agreement with the findings of the Nijmegen PSA [85].

The calculation of $p p$ scattering including Coulomb interactions to all orders consists of dressing the bubble diagrams in Fig. 2 with Coulomb exchanges 84,86]. This gives rise to integrals of the type (59) but with the free Schrödinger propagator (43) replaced by the Coulomb propagator

$$
G_{0 C}(l ; k)=-\frac{2 \pi \alpha m_{N} / l}{\exp \left(\alpha m_{N} / l\right)-1} \frac{m_{N}}{l^{2}-k^{2}-i \epsilon} .
$$

Dressing of the external legs gives rise to the usual Sommerfeld factor. In leading order, the 
amplitude can still be summed as a geometric series in terms of a single bubble integral. In addition to the linear cutoff dependence present even in the absence of electromagnetic interactions, a new cutoff dependence $\alpha m_{N}^{2} \ln \left(\Lambda / \alpha m_{N}\right) / \pi$ appears. This requires a new leading-order, no-derivative four-nucleon interaction

$$
C_{0 e m}^{(R)}=\frac{4 \pi \alpha}{\aleph^{2}} \gamma_{0 e m}
$$

to be added to $C_{0}^{(R)\left({ }^{1} S_{0}\right)}$, which can be fitted to the difference $a_{p p}-a_{n n}$. In this EFT the splitting in scattering lengths cannot be calculated without matching to the underlying theory. Effective-range - but no vacuum polarization - effects were considered in Refs. 86], where it was shown that to subleading order $r_{p p}=r_{n n}$. Effects of vacuum polarization and two-photon exchange are discussed in Refs. 87,88.

Moral. The modern EFT method applied to the problem of short-range forces is, after renormalization, completely equivalent to the ancient effective range expansion, and to the methods of pseudopotentials and boundary conditions. Why, then, bother with the EFT method? The gain is indeed marginal for the problem at hand, but there are advantages to a field-theoretical framework when other processes in the same energy scale are considered, and we want to treat them all consistently, free of off-shell ambiguities. (We will see an example in Sect. У.) Yet the gain is potentially much more significant in more general cases where there are other, lighter degrees of freedom that generate longer-range forces. Symmetries are easily incorporated in Lagrangian field theory. The problem considered in this Section has very weak symmetry constraints. In more interesting problems, such as the $N N$ system at energies comparable to the pion mass, the role of symmetry is more important, and the EFT method more convenient than the ancient techniques. We turn to this case next.

\section{B. Low energies}

As $Q$ approaches $m_{\pi}$, it becomes increasingly difficult to account for pion exchange as a short-range effect. As we further increase momenta past $Q \sim m_{\pi}$, we have to include an explicit pion field and build up all its interactions allowed by symmetries. The important new element is chiral symmetry, as discussed in Sect. IIq. Adding pions will generate all sorts of non-analytic contributions to the amplitude. For $Q \lesssim m_{\pi}$, pions need not but can be introduced explicitly, bringing non-analytic terms to $k \cot \delta$. However, these pionic terms can be expanded in a power series, and none of the terms with powers higher than the retained contact terms can be taken seriously, as neglected higher-order contact interactions could produce contributions of similar size. (Similar remarks hold for deuteron properties, as the deuteron is so dilute that it is even within the realm of the pionless EFT.) Nonetheless, one might hope to include at least part of the pion effects in this (uncontrolled) way. Indeed, lowenergy properties seem to improve and cutoff dependence to decrease if one-pion exchange (OPE) is added to the contact potential at $O\left(Q / M_{n u c}\right)(92)$, which is then iterated to all orders 88]. We want to go further and devise a rationale for a controlled expansion in the presence of pions, valid for momenta $Q \sim M_{n u c}$. The issue arises immediately of how 
to estimate the size of pion contributions. One needs to find (i) the importance of pion exchange relative to short-range interactions; (ii) how iterated OPE compares to OPE.

The OPE contribution to the $N N$ amplitude is, as we will see in more detail below, proportional to $Q^{2} / M_{n u c}^{2}\left(Q^{2}+M_{n u c}^{2}\right)$. On the other hand, as we have just seen, leading short-range effects are proportional to $4 \pi / M_{Q C D} \aleph$. Thus, as long as $\aleph \lesssim M_{N N}$ with $M_{N N} \sim 4 \pi M_{n u c}^{2} / M_{Q C D}$, OPE should be suppressed compared to the leading contact interaction by $\aleph / M_{N N}$. Using power counting arguments again, once-iterated pion exchange can give a contribution $\sim\left(Q^{2} / M_{n u c}^{2}\left(Q^{2}+M_{n u c}^{2}\right)\right)^{2}\left(M_{Q C D} Q / 4 \pi\right)$, and it also looks like a small correction to OPE as long as $Q \lesssim M_{N N}$. Of course, as in the lower-energy EFT, we expect all contributions to higher- $l$ partial waves to be perturbative at least up to momenta $\sim(2 l+1) M_{n u c}$. With such estimates, we conclude that at sufficiently small $Q$ pions might be treated perturbatively. The suggestion that pions can be profitably treated as perturbative even in $S$ waves and a power counting to support it were made in Ref. [77 in connection with a more complicated formalism, but details have not been published. Ref. 600 gave the first clear implementation of these ideas.

It is important to find out whether this can be done for momenta above $m_{\pi}$. Only if $M_{N N}$ $\gtrsim m_{\pi}$ are perturbative pions an improvement over the effective range expansion. Moreover, the full power of chiral symmetry in constraining pion interactions relies on an ordering of chiral-symmetry-breaking effects in powers of $m_{\pi}^{2}$. Including pions perturbatively brings terms that are a priori suppressed only by powers of $m_{\pi} / M_{N N}$. Chiral symmetry will be really useful only if this ratio is smaller than 1 . The problem is that $M_{N N}$ is not obviously larger than $M_{n u c}$; whether or not it actually is depends on dimensionless numbers that cannot be accounted for with dimensional analysis alone. The issue of the range of validity of the EFT with perturbative pions can only be settled by explicit calculation of dimensionless factors and comparison with precise observed quantities, this being done to sufficiently high order that a significant number of pion effects is tested.

Let us thus assume $\aleph<M_{N N}, Q<M_{N N}$, and $m_{\pi}<M_{N N}$. A simple power counting can be done by taking $Q \sim \aleph \sim m_{\pi}<M_{N N}$, and counting powers of the light scale $Q$. This is a direct extension of the power counting in the pionless EFT in Sect. IVA. In particular, the scaling of the contact operators is assumed to be the same as before with $M=M_{N N}$, and thus their ordering is unchanged. Because of chiral symmetry, each insertion of a pion exchange brings a factor of $Q / M_{N N}$. Electromagnetic interactions can be treated much as in the previous section.

If $M_{N N}$ is sufficiently high, we need to include the delta isobar as well. Beyond contact interactions considered previously in Eq. (92), and the pion-nucleon interactions in the one-nucleon sector shown in Sect. [1], there are new interactions involving four fermion fields where at least one is a delta, and involving four fermion fields and pions. For example [15, 17,

$$
\begin{gathered}
\mathcal{L}^{(0)}=-D_{T} N^{\dagger} \boldsymbol{t} \vec{\sigma} N \cdot\left(N^{\dagger} \boldsymbol{T} \vec{S} \Delta+\text { h.c. }\right)+\ldots \\
\mathcal{L}^{(1)}=-D_{1} N^{\dagger} N N^{\dagger} \boldsymbol{t} \cdot \vec{\sigma} \cdot \overrightarrow{\boldsymbol{D}} N-D_{2} \overrightarrow{\boldsymbol{D}} \cdot\left(N^{\dagger} \boldsymbol{t} \vec{\sigma} N \times N^{\dagger} \boldsymbol{t} \vec{\sigma} N\right)+\ldots \\
\mathcal{L}^{(2)}=m_{\pi}^{2}\left(1-2 D^{-1} \frac{\boldsymbol{\pi}^{2}}{F_{\pi}^{2}}\right)\left(C_{2 q m}^{(S)} N^{\dagger} N N^{\dagger} N+C_{2 q m}^{(T)} N^{\dagger} \vec{\sigma} N \cdot N^{\dagger} \vec{\sigma} N\right)+\ldots
\end{gathered}
$$



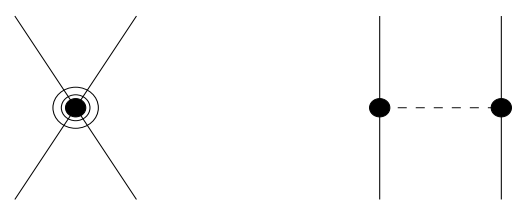

FIG. 7. Insertions at $O\left(Q / M_{N N}\right)$ in the perturbative-pion EFT, to be substituted for the circled dot in the second line of Fig. 3. A dashed line represents a pion, a heavy dot a derivative and a heavy dot with two circles two derivatives or powers of the pion mass.

Here $D_{T}, D_{i}$ and $C_{2 q m}^{(S, T)}$ are undetermined constants of mass dimensions $-2,-3$ and -4 respectively. "..." above stand for interactions involving more delta fields. So far investigations in this EFT have integrated out the delta isobar. As in the one-nucleon sector, this amounts to omitting the explicit-delta interactions and replacing the coefficients of nucleonic interactions by new ones that scale with factors of $1 / \delta m$. For example, Eq. (100) then involves new coefficients $d_{i}$ with

$$
d_{1}=D_{1}-\frac{4 D_{T} h_{A}}{9 \delta m}+\ldots, \quad d_{2}=D_{2}+\frac{2 D_{T} h_{A}}{9 \delta m}+\ldots
$$

The amplitude. The leading-order $N N$ amplitude coincides with that in the lowermomentum EFT, represented by the first line of Fig. 3 and by $T_{o s}^{(0)}$ (the $C_{0}$ and $i k$ terms) in Eq. (64): it is the iteration of chirally-symmetric, non-derivative contact interactions. At this order there are contributions only in the two $S$-wave channels. Subleading terms, of $O\left(Q / M_{N N}\right)$ relative to leading, are constructed in a direct extension of subleading terms of the lower-momentum EFT, second line of Fig. 3 and $T_{o s}^{(1)}$ (the $C_{2}$ term) in Eq. (64). The difference is that now besides two-derivative contact interactions we also insert one-pion exchange and a non-derivative contact interaction that breaks chiral symmetry explicitly. In other words, besides the $C_{2}$ term in Eq. (58) we need to consider one insertion of

$$
V=-\left(\frac{2 g_{A}}{F_{\pi}}\right)^{2} \boldsymbol{t}_{1} \cdot \boldsymbol{t}_{2} \frac{\vec{\sigma}_{1} \cdot \vec{q} \vec{\sigma}_{2} \cdot \vec{q}}{\vec{q}^{2}+m_{\pi}^{2}}+m_{\pi}^{2}\left(C_{2 q m}^{(S)}+C_{2 q m}^{(T)} \vec{\sigma}_{1} \cdot \vec{\sigma}_{2}\right)
$$

- see Fig. 7. Here we have neglected the energy transferred in OPE as it is a higher order effect. When Eq. (103) is inserted within loops with $T_{o s}^{(0)}$, new divergences arise that are $\propto m_{\pi}^{2}$. These divergences can be absorbed in the $C_{2 q m}$ 's, giving rise to renormalized $C_{2 q m}^{(R)}$ 's. Both $C_{2}$ and $C_{2 q m}$ contact interactions also only contribute to $S$ waves. The tensor operator from pion exchange, on the other hand, introduces mixing between ${ }^{3} S_{1}$ and ${ }^{3} D_{1}$ waves. To this order, the ${ }^{3} S_{1}-{ }^{3} D_{1}$ mixing parameter and the ${ }^{3} D_{1}$ phase shift are predicted in terms of pion parameters (and the short-ranged parameters that can be determined from the ${ }^{3} S_{1}$ phase shift).

A calculation of the $N N$ system to this order was first carried out in Ref. [60] in the PDS subtraction scheme, and repeated with a coordinate space cutoff in Ref. [89]. One finds in the ${ }^{1} S_{0}$ channel [60,89 91]

$$
k \cot \delta^{\left({ }^{1} S_{0}\right)}=-\frac{1}{\left.a_{2}^{(1} S_{0}\right)}+\frac{r_{2}^{\left(1 S_{0}\right)}}{2} k^{2}+\mathcal{S}^{\left({ }^{1} S_{0}\right)}(k)+O\left(k^{2} / \Lambda, m_{\pi}^{2} / \Lambda, 1 / a_{2}^{\left({ }^{1} S_{0}\right) 2} \Lambda\right)
$$


where

$$
\begin{gathered}
a_{2}^{\left(1 S_{0}\right)}=\frac{m_{N} C_{0}^{(R)}}{4 \pi}\left[1+\frac{g_{A}^{2} m_{\pi} m_{N}}{2 \pi F_{\pi}^{2}}\left(1-\frac{1}{2} \frac{4 \pi}{m_{\pi} m_{N} C_{0}^{(R)}}\right)+\frac{m_{\pi}^{2} C_{2 q m}^{(R)}}{C_{0}^{(R)}}\right], \\
r_{2}^{\left({ }^{1} S_{0}\right)}=\frac{16 \pi C_{2}^{(R)}}{m\left(C_{0}^{(R)}\right)^{2}}+\frac{g_{A}^{2} m_{N}}{2 \pi F_{\pi}^{2}}\left[1-\frac{8}{3} \frac{4 \pi}{m_{\pi} m_{N} C_{0}^{(R)}}+2\left(\frac{4 \pi}{m_{\pi} m_{N} C_{0}^{(R)}}\right)^{2}\right],
\end{gathered}
$$

and

$$
\begin{aligned}
\mathcal{S}^{\left({ }^{1} S_{0}\right)}(k)=\frac{g_{A}^{2} m_{\pi}^{2} m_{N}}{4 \pi F_{\pi}^{2}} & {\left[\frac{1}{4} \ln \left(1+4 \frac{k^{2}}{m_{\pi}^{2}}\right)-\frac{k^{2}}{m_{\pi}^{2}}+2 \frac{4 \pi}{m_{\pi} m_{N} C_{0}^{(R)}}\left(\frac{m_{\pi}}{2 k} \arctan \left(\frac{2 k}{m_{\pi}}\right)-1+\frac{4 k^{2}}{3 m_{\pi}^{2}}\right)\right.} \\
& \left.-\left(\frac{4 \pi}{m_{\pi} m_{N} C_{0}^{(R)}}\right)^{2}\left(\frac{m_{\pi}^{2}}{4 k^{2}} \ln \left(1+4 \frac{k^{2}}{m_{\pi}^{2}}\right)-1+\frac{2 k^{2}}{m_{\pi}^{2}}\right)\right] .
\end{aligned}
$$

This is in the form of an effective range expansion supplemented by a non-analytic "shape function" generated by pion exchange. Similar expressions hold for $k \cot \delta^{\left({ }^{3} S_{1}\right)}$, and analytic formulas can also be derived for $\varepsilon_{1}$ and $\delta^{\left({ }^{3} D_{1}\right)} 60,89$, 91.

To this order, there are three unknown parameters in the ${ }^{1} S_{0}$ channel $\left(C_{0}^{\left({ }^{1} S_{0}\right)}, C_{2}^{\left({ }^{1} S_{0}\right)}\right.$, $\left.C_{2 q m}^{\left({ }^{1} S_{0}\right)}\right)$, and three $\left(C_{0}^{\left({ }^{3} S_{1}\right)}, C_{2}^{\left({ }^{3} S_{1}\right)}, C_{2 q m}^{\left({ }^{3} S_{1}\right)}\right)$ in the ${ }^{3} S_{1}$ channel. Note, however, that the amplitude to this order can be written as a function of $C_{0}+m_{\pi}^{2} C_{2 q m}$ for it is this combination that appears in the potential (92) $+(103)$. Only at higher orders will $C_{2 q m}$ contribute new terms through pion loops. To subleading order, then, the separate dependence of observables on $C_{0}$ and $C_{2 q m}$ is an artifact of perturbation theory. The difference between treating $C_{2 q m}$ perturbatively and non-perturbatively is an indication of the size of higher-order corrections.

A fit to the Nijmegen $S$-wave phase shifts [78] was done in Ref. [60] using the amplitude derived in the PDS scheme. As described in Sect. IVA, the theory can then be written in terms of running couplings such as $C_{2 q m}(\mu)$. The scattering lengths and effective ranges can be used to determine $C_{0}$ and $C_{2}$ with a choice of $C_{2 q m}$. Choosing $C_{2 q m}\left(m_{\pi}\right)=0$, the predicted ${ }^{1} S_{0}$ phase shifts deviate significantly from the Nijmegen PSA at momenta of order $m_{\pi}$. The amplitude with three free parameters in each $S$-wave gives a much better fit for momenta below $200 \mathrm{MeV}$; the predicted ${ }^{3} S_{1}-{ }^{3} D_{1}$ mixing parameter $\varepsilon_{1}$ and the ${ }^{3} D_{1}$ phase then come out reasonably well - see Fig. 8 [60].

Note that to this order other waves are determined as well. For example, $P$ waves are given by OPE, and indeed it is well-known that the sizes of triplet scattering volumes is roughly reproduced by OPE [92]. Similarly, it is been part of nuclear folklore that high partial waves are mostly due to perturbative pion effects 92]. The various perturbative contributions from pion exchanges to $D$ and higher waves was examined in detail in Ref. [93, up to sub-subleading order. The $D$ wave cannot not be described well above laboratory energies of $\sim 50 \mathrm{MeV}$ without short-range interactions, but higher waves get progressively better described by perturbative pions, as expected.

Schrödinger formulation. The EFT with perturbative pions can be recast in terms of the Schrödinger equation in coordinate space. In Ref. 89 it was shown that the above subleading amplitude can be obtained by a Born expansion on the Yukawa part of the pion 

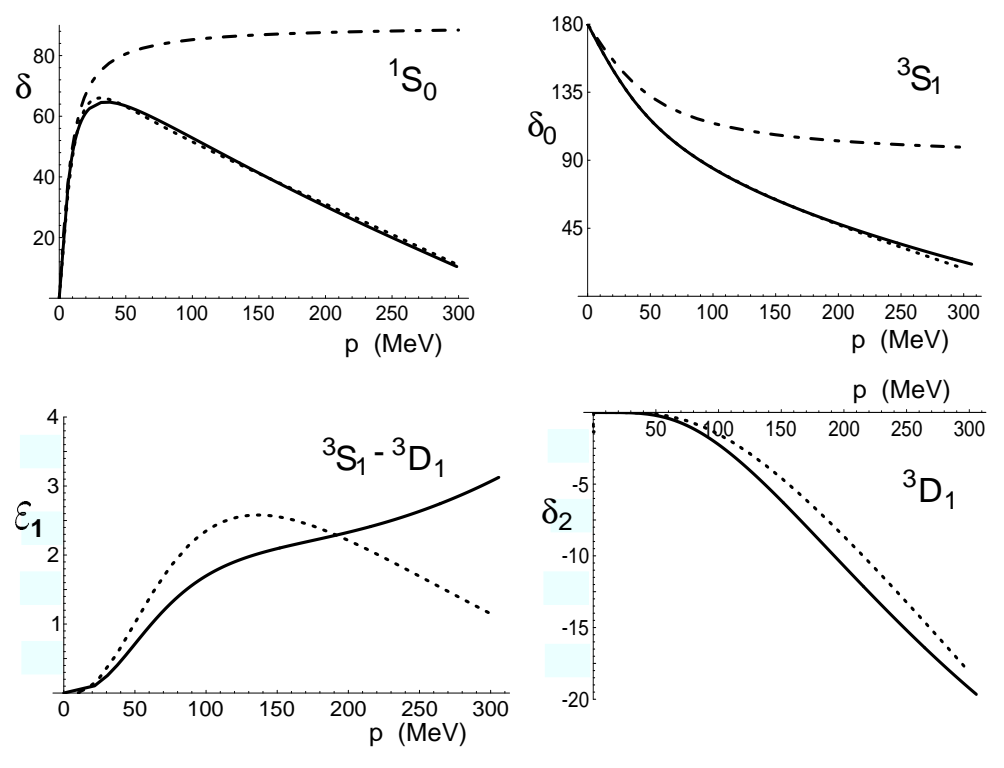

FIG. 8. ${ }^{1} S_{0},{ }^{3} S_{1}$, and ${ }^{3} D_{1} N N$ phase shifts and $\varepsilon_{1}$ mixing angle in degrees as functions of center-of-mass momentum in MeV: EFT in leading (dot-dashed) and subleading (dashed line) orders; and Nijmegen PSA (solid line). Figure courtesy of M. Savage.

potential at distances $r \geq R \sim 1 / \Lambda$. The wave-function can be written as $u(r)=\sum_{i=0}^{\infty} u_{i}(r)$, where (i) the leading term is a solution of the free Schrödinger equation, $u_{0}=\sin \left(k r+\delta_{0}\right)$, with $k \cot \delta_{0}$ determined by the contact interactions; and (ii) higher-order terms satisfy $u_{i}(R)=0 . u_{1}(r)$ can be expressed in terms of simple integrals involving the long-range part of the potential.

Range of the theory and regularization issues. From the expressions for the amplitude shown above, it seems that pion effects are associated with the scale $M_{N N} \sim$ $4 \pi F_{\pi}^{2} / g_{A}^{2} m_{N} \simeq 300 \mathrm{MeV}$ [60. If so, the expansion parameter is $\sim m_{\pi} / M_{N N} \simeq 0.5$. The scattering length (105) is formally of the form $\sim \aleph+O\left(Q^{2} / M_{N N}\right)$. $C_{0}^{(R)}$ can be estimated in leading order using Eq. (67) and then used in Eqs. (106) and (107) to estimate the relative size of pion contributions. For example, OPE is responsible for about half of the effective range (106). Note, however, that this simple-minded procedure is not without its caveats. OPE contributions to Eq. (105) are not small. They include a contribution which is non-analytic in the quark mass; this term clearly shows not only that the expansion is in $m_{\pi} / M_{N N}$ even at $k=0$, but also that dimensionless numerical factors can be dangerous, since $g_{A}^{2} m_{\pi} m_{N} / 2 \pi F_{\pi}^{2} \simeq 0.9$. Nevertheless, $C_{2 q m}$ can be adjusted numerically to compensate, maintaining the lowest-order mostly unchanged. The reasonable success observed in the lowpartial-wave phase shifts suggests that dimensionless numbers do indeed conspire to increase $M_{N N}$. A number of recent papers have attempted to provide a more precise estimate of $M_{N N}$. The issue of a practical test of convergence has been tied to specific regularization schemes employed in calculations.

In Ref. 94 the ${ }^{1} S_{0}$ fit has been reexamined with both PDS and a subtraction at $p^{2}=$ $-\mu^{2}$. Significant differences were found in the phases for $Q \gtrsim \mu$ depending on whether $C_{2}$ and $C_{2 q m}$ were included perturbatively or non-perturbatively. This led the author of Ref. 
94 to conclude that pions can be included perturbatively only for external momenta well below the pion mass. In Ref. [95] a similar conclusion was reached through a Lepage error analysis. In Ref. [96 it is argued that this strong sensitivity to $\mu$ arises from the fact that the subleading amplitude in PDS is not $\mu$ independent, so that the parameters that satisfy renormalization group equations cannot give a good fit to phase shifts. Pion exchange gives contributions that do not vanish in the chiral limit, and introduces subleading but potentially significant dependence on $\mu$. It was shown that this $\mu$ dependence can be ameliorated by splitting $C_{0}$ into two pieces, $C_{0}=C_{0[0]}+C_{0[2]}$, where $C_{0[0]}$ is treated non-perturbatively while $C_{0[2]} \sim m_{\pi}^{2} C_{2 q m} \ll C_{0[0]}$ is treated as a first-order correction. The amplitude in subleading order is then a function of $C_{0[2]}+m_{\pi}^{2} C_{2 q m}$. An improved subtraction scheme ("OS") designed to minimize the $\mu$ dependence of the perturbative expansion was also introduced, and the subleading amplitude computed. Fits to ${ }^{1} S_{0}$ and ${ }^{3} S_{1}$ phase shifts for momenta between 7 and $100 \mathrm{MeV}$ - fits that are weighted towards low momenta - are then reasonable, at the same time producing good values for the scattering lengths. The freedom of fitting $C_{2 q m}$ can enforce smallness of the full subleading contribution. The authors of Ref. [96] conclude that there are no obstructions to using perturbative pions for external momenta larger than the pion mass.

Refs. 90,91] point out that the shape functions are completely determined by pion exchange. For momenta below the pion mass, the shape functions can be expanded in powers of $k^{2}$, and effective range parameters other than scattering lengths and effective ranges are likewise determined. For example, in either $S$-wave channel $v_{2}^{(n)(0)} \sim 1 / m_{\pi}^{n-2} M_{N N}$. Such relations can be viewed as a one-pion-saturation model 42 of the parameters of the verylow-energy EFT of Sect. IVA. One can see that the perturbative-pion EFT provides no explanation for the fine-tuning in the scattering lengths, but does provide higher-order effective range parameters which scale approximately as $1 / M_{n u c}^{n-1}$ as expected from dimensional analysis. Note that these parameters blow up in the chiral limit as a consequence of the long range of pion interactions. If the perturbative-pion EFT can be shown to hold for $Q \gtrsim m_{\pi}$, then these predictions can be elevated to bona fide low-energy theorems. On the other hand, if the perturbative-pion EFT holds only for $Q \lesssim m_{\pi}$, contact parameters showing up at the same order render the pion predictions no better than an order-of-magnitude estimate. The shape function and higher effective range parameters look like good testing grounds of the convergence of the expansion as - contrary to $C_{2 q m}$ in $a_{2}$ - there are no parameters that can be fine-tuned to balance OPE effects.

In Ref. 90] the shape functions were expanded in a series $k^{2}$. The mixing parameter $\varepsilon_{1}$ was expanded as well,

$$
\varepsilon_{1}=\sum_{i=1}^{\infty} g^{(i)} k^{2 i+1}
$$

although this is expected to breakdown at very low momenta $\sim$; in view of Eq. (93) it is no surprise that $g_{i} \sim 1 / \aleph^{2 i-1} m_{\pi} M_{N N}$. The result of a comparison between perturbative pion predictions and values of effective range (66) and $\varepsilon_{1}$ (108) parameters extracted from the Nijmegen PSA [78] is in Table [II] [90]. Because of the dramatic failure of these predictions the authors of Ref. [90] suggest that the scale of the physics left out from this perturbativepion EFT is not much larger than $m_{\pi}$. They further suggest that successes of this EFT 
TABLE III. Parameters of analytic expansions of $k \cot \delta$ in the ${ }^{1} S_{0}$ and ${ }^{3} S_{1}$ channels and of the ${ }^{3} S_{1}-{ }^{3} D_{1}$ mixing angle $\varepsilon_{1}$ predicted by the EFT with perturbative pions compared to values obtained from the Nijmegen PSA.

\begin{tabular}{|c|c|c|c|c|c|c|c|c|c|}
\hline & \multicolumn{3}{|c|}{${ }^{1} S_{0}$} & \multicolumn{3}{|c|}{${ }^{3} S_{0}$} & \multicolumn{3}{|c|}{${ }^{3} S_{1}-{ }^{3} D_{1}$} \\
\hline & $\overline{v_{2}^{(2)(0)}}$ & $v_{2}^{(3)(0)}$ & $v_{2}^{(4)(0)}$ & $\overline{v_{2}^{(2)(0)}}$ & $v_{2}^{(3)(0)}$ & $v_{2}^{(4)(0)}$ & $\overline{g^{(1)}}$ & $g^{(2)}$ & $\overline{g^{(3)}}$ \\
\hline$\overline{\mathrm{EFT}}$ & -3.3 & 17.8 & -108 & -0.95 & 4.6 & -25 & 3.9 & -86 & 1800 \\
\hline PSA & -0.48 & 3.8 & -17 & 0.04 & 0.67 & 4.0 & 1.7 & -26 & 220 \\
\hline
\end{tabular}

are coming from the expansion in $\aleph / M_{N N}$, with the failure of the expansion in $m_{\pi} / M_{N N}$ disguised by counterterms. They attribute the good fits of Fig. \& to the fact that any theory with free parameters consistent with the effective range expansion can reproduce the rapid rise at low $k$ and the subsequent slow decrease of the $S$-wave phase shifts. However, using the energy-independent Nijmegen analysis, the authors of Ref. [96] have argued that the uncertainty in $v_{2}^{(2)(0)}$ is too large to make a definite test of the OPE predictions. They claim that uncertainties in the $v_{2}^{(n)(0)}$ 's from the energy-dependent Nijmegen analysis are unrealistically small, and question whether low-energy data alone can be used to determine these parameters. Ref. 91] has reexamined these issues directly in terms of the shape function at finite energy. It is shown that the shape function in the ${ }^{3} S_{1}$ channel is welldetermined by the PSA: at the deuteron pole, the shape function is close to zero to a good precision resulting from the accurately known values of $B_{2}, a_{2}$, and $r_{2}$; at positive energies the finite-energy phase shifts are also need to extract $\mathcal{S}$. It is found that OPE saturation fails again way beyond error bars. Because $\mathcal{S}$ is small compared to $k \cot \delta$, Ref. [91] points out that one has to include relativistic and isospin-breaking effects in the extraction of $\mathcal{S}$. This is a little unsettling, however, as these are higher-order effects that were not included in the EFT estimate.

Although work done so far has gone some distance in assessing the validity of perturbative pions, this remains one of the most important open questions for the EFT approach in nuclear systems. Work along this line requires the evaluation of higher-order effects. At sub-subleading order we encounter new pion exchanges: both non-static (or radiative) OPE and once-iterated OPE. Radiative pion diagrams have very recently been studied in Ref. 97. It is suggested that the size of these graphs can be estimated counting powers of $\sqrt{m_{\pi} m_{N}}$, yet mysterious cancellations have been found that render the sum of leading non-vanishing nonstatic pion diagrams smaller than expected. The role of deltas has not yet been examined. Particularly in diagrams with non-static pions, integrating deltas out should significantly limit the range of the EFT. So far there is also no explicit calculation of the dimensionless factors that are involved in once-iterated OPE, and of the sizes of sub-subleading contact interactions. Work is in progress in this direction 98 and a better determination of the range of validity of this EFT should be available soon. As we will see in Sect. VA, this is pivotal in assessing the usefulness of this EFT for nuclei other than the deuteron. In Sects. $\mathrm{VA}$ and VIA it will be evident that this EFT allows a much simpler treatment of nuclear systems than afforded by traditional methods. 
Finally, note - in complete analogy to the extensive discussion in Sect. IVA that if pions can be treated perturbatively then little error is made in iterating them to all orders. There might even be something to gain if one is lucky that short-range effects are small because of unknown dimensionless factors. The procedure of including pions in a potential that is then iterated to all orders had actually been carried out before any of the developments reviewed in the present section. We turn to this next.

\section{Moderate energies}

We want to extend the EFT approach to momenta $Q \gtrsim M_{N N}$. How this is to be done depends critically on the value of $M_{N N}$. At this scale pions become non-perturbative. If $M_{N N}$ is as large as $M_{Q C D}$ the task is daunting as there is presently no rationale for a controlled expansion in the presence of massive degrees of freedom such as the rho. On the other hand, if $M_{N N} \ll M_{Q C D}$, we might hope to improve the expansion of the previous section by a controlled resummation of selected terms that go as $Q / M_{N N}$. If $M_{N N} \lesssim$ $300 \mathrm{MeV}$, the lack of known particle thresholds there suggests that the resummation could involve only pions. For $M_{N N} \gtrsim 300 \mathrm{MeV}$ delta isobars probably need to be included as well.

In fact, naive dimensional analysis alone suggests that the leading effect of pion exchange should be iterated to all orders for $Q \sim M_{n u c}$. This is because numerically $M_{Q C D} \sim 4 \pi M_{n u c}$, and thus $M_{N N} \sim M_{n u c}$. The new element is that $\mathrm{OPE}$ is $\sim 1 / M_{n u c}^{2} \sim 4 \pi / M_{Q C D} M_{n u c}$. Because OPE has short-range components, it is natural to assume that they set the scale for , $\sim \sim M_{n u c} \ll M_{Q C D}$. Part of the unnaturalness of $\aleph$ is thus explained. (It is still mysterious why $\aleph<M_{n u c}$; some cancellation between short and long-range effects is necessary to enforce this.) This suggests a power counting might be meaningful in which we take $Q \sim M_{N N} \sim \aleph \sim M_{n u c} \sim M_{Q C D} / 4 \pi$. Now, arguments analogous to those in Sect. IVA get complicated to the point that they can no longer be carried out analytically in detail. Nevertheless they are still valid: a loop containing a reducible intermediate state still contributes $M_{Q C D} Q / 4 \pi$, so adding no-derivative contact interactions $\left(\sim 4 \pi / M_{Q C D} \aleph\right)$ or static OPE $\left(\sim 1 / M_{n u c}^{2}\right)$ to a graph amounts to a power of $Q / \aleph \sim Q / M_{n u c}$. In order to describe $Q \sim M_{n u c}$ we have to sum all graphs with the leading potential, no-derivative contact interactions plus static OPE. All other contributions would come as corrections. This power counting is due to Weinberg [10,13], but many of its elements had been anticipated by Friar 99,100.

The question has been raised, whether such a power counting can be carried out consistently. The problem is that once OPE is iterated to all orders, renormalization of the theory becomes quite different from the perturbative-pion EFT. Perturbative arguments have been given 60,96] to support the claim that Weinberg's power counting is not consistent. Ref. [60] points out that a diagram with OPE between two $C_{0}$ interactions requires the $C_{2 q m}$ counterterm, as we have seen in the previous section. Ref. 96] finds that thrice-iterated OPE and diagrams with twice-iterated OPE plus one $C_{0}$ interaction require a $C_{2}$ counterterm. This implies that alleged higher-order contact interactions might get contaminated by powers of $M_{Q C D} / M_{n u c}$ in the numerator, to the point that they are required to appear in leading order. If this is a feature of an infinite number of contact interactions, there is no ordering and this EFT is doomed. However, it has been known in the context of the 
Schrödinger equation that perturbative arguments of this type are not in general reliable for singular potentials, as is the case for OPE. The perturbative expansion might have a cut starting at $g_{A}^{2} / F_{\pi}^{2}=0$; insistence on a $g_{A}^{2} / F_{\pi}^{2}$ expansion would then reflect itself on different orders offering correlated contributions to counterterms, each bringing powers of $M_{Q C D} / M_{n u c}$, yet resulting in a much better behaved sum. This conjecture ultimately has to be decided on the basis of actual calculations. We will assume it to be correct, fit phase shifts and then examine the cutoff dependence and convergence of the expansion. If the conjecture is correct, then we should find that this EFT has a range of validity greater than $M_{N N}$. Although less familiar to those raised with perturbative calculations, this procedure is quite analogous and no less justified than the methods being used to assess the range of validity of the perturbative-pion EFT. We are going to see that there is evidence that renormalization is being performed correctly, as cutoff dependence turns out to be weak.

The power counting is most easily done for the potential $V$. An arbitrary contribution to the two-nucleon potential can be represented by a two-nucleon irreducible Feynman diagram, that is, with $A=2$ continuous nucleon lines and at least one pion or delta isobar in intermediate states. Effects from the scale $M_{Q C D}$ are lumped in the vertices, and by restricting ourselves to the potential we exclude the scale $Q^{2} / M_{Q C D}$ from energy denominators. Since the only explicit scale is $Q$, the $\chi \mathrm{PT}$ counting (16) applies directly if we put $A=2[10,13]$,

$$
\nu=2 L+\sum_{i} V_{i} \Delta_{i}
$$

In this way the benefits of chiral symmetry are extended to nuclear physics: since both $L$ and $\Delta_{i}$ are bounded from below $\left(L \geq 0, \Delta_{i} \geq 0\right), \nu \geq \nu_{\min }=0$. Note that this counting is a direct generalization of the counting (86) of the pionless EFT, where the potential has no loops and a single vertex of index $\Delta=d$. Isospin violation will be considered shortly.

We assume implicitly that once $M_{n u c}\left(F_{\pi}, m_{\pi}\right.$, possibly $\delta m$, and, neglecting fine-tuning, $\aleph)$ have been accounted for, all other dimensionful quantities are given by $M_{Q C D}$. Since we also assume that no enhancements appear from the iteration of the potential, the scaling of contact interactions is determined by pion exchange in the potential: as in the case of a single nucleon, we expect additional $Q^{2}$ to be accompanied by $M_{Q C D}^{2}$, so that

$$
C_{2 n}^{\cdots(R)}=\frac{4 \pi}{M_{Q C D}^{2 n+1} M_{n u c}} \gamma_{2 n}^{\cdots},
$$

with the $\gamma_{2 n}^{(\ldots)}$ ) s dimensionless factors of $O(1)$.

Thanks to infrared enhancement, reducible diagrams have to be treated differently than the potential. The leading-order potential is iterated to all orders. Corrections to the leading potential do not need to be iterated to all orders. Yet, as we have seen in Sect. IV A, they can be iterated with small error as long as one uses a regularization with a cutoff mass $\Lambda \sim M_{Q C D}$; for the two-nucleon system, this is equivalent to solving the Schrödinger equation with the potential $V$. A potential up to a certain order ensures that the amplitude is correct to the same order. Of course, even if our assumptions about the scaling of interactions in this EFT are incorrect, results from the iteration of the potential with a sufficient number of interactions automatically include all results of perturbative pions discussed in the previous section. 
Traditionally, potential models have been plagued by problems of principle, such as the form of meson-nucleon interactions (for example pseudoscalar vs. pseudovector pion coupling), renormalization issues, absence of a small expansion parameter, etc. Because the EFT potential includes explicitly the exchange of only pions, all these problems can be resolved. As we have seen in Sect. IIq, because pions are pseudo-Goldstone bosons, the form of their interactions with nucleons is determined by the pattern of chiral symmetry and its breaking. (For example, if the EFT were formulated relativistically, pseudovector coupling would be preferred, as insistence on pseudoscalar form would demand new seagull vertices to ensure "pair suppression" 99]). Renormalization can be performed as all interactions consistent with symmetries have been included. And the power counting (109) for the EFT potential implies that diagrams with an increasing number of loops $L$-and, in particular, with increasing number of exchanged pions - should be progressively less important. Clearly, the EFT potential can be thought of as a low-energy approximation to standard potential models, although this can only be taken in an average sense. The scope of an EFT potential for systems with at least two mass scales has been examined in toy models. Ref. [70] considers the case of a short-range potential supplemented by a Coulomb potential. It exemplifies through an efficient error analysis how the approximation of contact interactions for the short-range potential improves the low-energy description systematically. The small role played by the cutoff $\Lambda$ can also be shown explicitly in this case. Similar conclusions hold in toy models where two nucleons interact via a light scalar with Yukawa coupling and a short-range potential [95, 101]. It has been shown that, even in the presence of a fine-tuning that produces a shallow $S$-wave bound state, the contact interactions obey Eq. (110), with $M_{Q C D}$ representing the heavy mass scale. (Ref. [95] further emphasizes the advantages of a fit to modified effective range parameters.) The EFT potential thus provides a QCD justification for some of the phenomenological successes of potential models.

Potential and amplitude. The Lagrangian for this EFT is the same considered before in the context of perturbative pions. A calculation of all contributions to the two-nucleon potential up to $\nu=\nu_{\min }+3$ was carried out in Refs. 12, 15, 16 using time-ordered perturbation theory. Some diagrams are shown in Fig. 9 .

In leading order, $\nu=\nu_{\min }$, the potential is simply static OPE and momentumindependent contact terms, that is, the first term in Eq. (103) and the first two in Eq. (92) [10]. This is obviously a very crude approximation to the $N N$ potential. It does allow us to see how energies of $O(10 \mathrm{MeV})$ arise from QCD: the average of the leading-order potential in coordinate space is roughly $\left\langle V_{2 N}^{(0)}\right\rangle \sim-g_{A}^{2} m_{\pi}^{3} / 4 \pi F_{\pi}^{2} \sim-10 \mathrm{MeV}$. However, it is known that the nuclear force has other sizable components, like a spin-orbit force, a strong short-range repulsion and an intermediate range attraction. These are all generated in the next orders: $\nu=\nu_{\min }+1$ corrections vanish due to parity and time-reversal invariance, but $\nu=\nu_{\min }+2$ corrections are several. First, there are short-range corrections; they come from one-loop pion dressing of the lowest-order contact interactions, and from four-nucleon contact interactions with two derivatives or two powers of the pion mass, displayed in Eqs. (92) and (103). It is easy to show that the result of loop diagrams amount to a simple shift of the contact parameters. In Refs. [12, 15, 16 the $C_{0}$ 's were redefined in order to reabsorb both loop contributions and the chiral-symmetry-breaking $C_{2 q m}$ 's, as to this order they cannot be separately determined from $N N$ data. Second, there are corrections to OPE; these come 


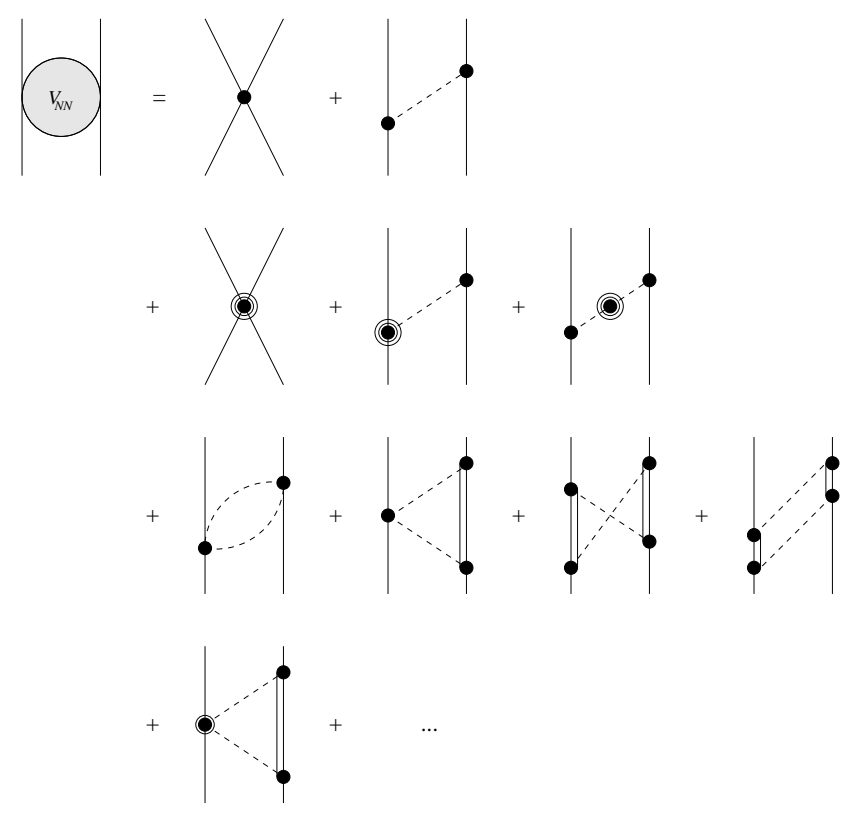

FIG. 9. Some time-ordered diagrams contributing to the two-nucleon potential $V_{N N}$ in the EFT. (Double) solid lines represent nucleons (and/or deltas), dashed lines pions, a heavy dot an interaction in $\mathcal{L}^{(0)}$, a dot within a circle an interaction in $\mathcal{L}^{(1)}$, and a dot within two circles an interaction in $\mathcal{L}^{(2)}$. First line corresponds to $\nu=\nu_{\min }$, second and third lines to $\nu=\nu_{\min }+2$, fourth line to $\nu=\nu_{\min }+3$, and "..." denote $\nu \geq \nu_{\min }+4$. All orderings with at least one pion or delta in intermediate states are included. Not shown are diagrams contributing only to renormalization of parameters.

from vertex dressing and from recoil upon pion emission:

$$
V_{\text {tree }}^{(2)}=-\frac{2 g_{A}}{F_{\pi}^{2}} \boldsymbol{t}_{1} \cdot \boldsymbol{t}_{2} \frac{\vec{\sigma}_{1} \cdot \vec{q} \vec{\sigma}_{2} \cdot \vec{q}}{\vec{q}^{2}+m_{\pi}^{2}}\left(A_{1} q^{2}+A_{2} k^{2}-2 g_{A} \frac{E-\frac{1}{4 m_{N}}\left(4 \vec{k}^{2}+\vec{q}^{2}\right)}{\sqrt{\vec{q}^{2}+m_{\pi}^{2}}}\right) .
$$

Again, here loop corrections have been absorbed in mass and coupling constant renormalization. Also, chiral-symmetry-breaking corrections to the $\pi N N$ coupling proportional to $m_{\pi}^{2}$ can be lumped in $g_{A}$, which then satisfies the Goldberger-Treiman relation without discrepancy. Third, there are two-pion exchange (TPE) diagrams built out of lowest-order $\pi N N$ and $\pi N \Delta$ interactions of Eq. (47). Denoting $\omega_{ \pm} \equiv \sqrt{(\vec{q} \pm \vec{l})^{2}+4 m_{\pi}^{2}}$, these loop diagrams can be expressed schematically as

$$
V_{\text {loop }}^{(2)}=\frac{\left(g_{A}, h_{A}\right)}{F_{\pi}^{4}}\left(1, \boldsymbol{t}_{1} \cdot \boldsymbol{t}_{2}\right) \int \frac{d^{3} l}{(2 \pi)^{3}}\left(\frac{1}{\omega_{ \pm}}, \frac{\vec{q}^{2}-\vec{l}^{2}}{\left(\omega_{ \pm}^{3}, \omega_{ \pm}^{2} \delta m\right)}, \frac{\left(\left(\vec{q}^{2}-\vec{l}^{2}\right)^{2}, \vec{\sigma}_{1} \cdot(\vec{q} \times \vec{l}) \vec{\sigma}_{2} \cdot(\vec{q} \times \vec{l})\right)}{\left(\omega_{ \pm}^{5}, \omega_{ \pm}^{4} \delta m, \omega_{ \pm}^{3}(\delta m)^{2}, \omega_{ \pm}^{2}(\delta m)^{3}\right)}\right) .
$$

At $\nu=\nu_{\min }+3$ a few more TPE diagrams appear, which involve the $\pi \pi N N$ seagull vertices from Eq. (48); again schematically,

$$
V_{\text {loop }}^{(3)}=\frac{\left(g_{A}^{2}, h_{A}^{2}\right) B_{i}}{F_{\pi}^{4}}\left(1, \boldsymbol{t}_{1} \cdot \boldsymbol{t}_{2}\right) \int \frac{d^{3} l}{(2 \pi)^{3}} \frac{\left(m_{\pi}^{2}\left(\vec{q}^{2}-\vec{l}^{2}\right),\left(\vec{q}^{2}-\vec{l}^{2}\right)^{2}, \vec{\sigma}_{1} \cdot(\vec{q} \times \vec{l}) \vec{\sigma}_{2} \cdot(\vec{q} \times \vec{l})\right)}{\left(\omega_{ \pm}^{4}, \omega_{ \pm}^{3} \delta m\right)} .
$$


To this order there are some relativistic corrections as well, but they are numerically small and have been neglected.

This potential has all the spin-isospin structure of phenomenological models, but its profile is determined by explicit degrees of freedom, symmetries, and power counting. The power counting suggests a hierarchy of short-range effects: $S$ waves should depend strongly on the short-range parameters $C_{0}$; contact interactions affect $P$-wave phase shifts only in subleading order, so their effect should be smaller and approximately linear; $D$ waves are affected by contact interactions only via mixing, while higher waves should be essentially determined by pion exchange. Chiral symmetry is particular influential in the TPE piece. The latter includes a particular form of terms previously considered by Brueckener and Watson [102], Sugawara and von Hippel [103], and Sugawara and Okubo [104, plus a few new terms. Those terms involving the $B_{i}$ 's and the deltas provide the only form of correlated TPE to this order, as graphs where pions interact in flight appear only in next order and should thus be relatively small. The sum of the $B_{1}$ term and the corresponding delta term (which give the $c_{3}$ of Eq. (54), related to the nucleon axial polarizability), is particularly important in providing an isoscalar central force. Not surprisingly, in the chiral limit these potentials behave at large separations as van der Waals forces.

Regularization and renormalization are necessary not only for the loops in the potential but also for the loops generated by the solution of the Schrödinger equation. For numerical convenience a smooth cutoff of the Gaussian type was used, and calculations performed with the cutoff parameter $\Lambda$ taking values 500, 780 and $1000 \mathrm{MeV}$. For each cutoff value a set of (bare) parameters was found that fits phase shifts below $100 \mathrm{MeV}$ laboratory energies. There are nine independent parameters stemming from contact interactions, although for the fit to $N N$ phase shifts we have also varied nine other, redundant parameters. OPE and TPE diagrams are completely determined by parameters accessible in pion-nucleon reactions, but because most were not known at the time they were also searched in the fit. A sample of the results for the lower, more important partial waves is presented in Fig. 10, together with phases from the Nijmegen PSA 78 — cf. Fig. 8. Quality of the fits is typical of other waves. Waves higher than $F$ were found to be mostly described well by pion exchange alone, as expected. Deuteron quantities are shown in Table IV — cf. Table III. (Electromagnetic quantities refer to the contributions from lowest-order $\gamma N N$ couplings only, not to a consistent calculation which would include sub-leading one- and two-nucleon effects.) The predicted $S$-wave scattering lengths (not used to constrain the fit) were found to be $a_{2}^{\left(1 S_{0}\right)} \simeq-15.0 \mathrm{fm}$ and $a_{2}^{\left(3 S_{1}\right)} \simeq 5.46 \mathrm{fm}$. Important central attraction comes from the $B_{i}$ 's and deltas, and indeed the central potential does resemble that from models that include $\sigma$ and $\omega$ meson exchange explicitly. Values for the parameters are listed in Ref. [16]. Reasonable values were found for quantities known at the time, for example $g_{A}=1.33$ (in agreement with the Goldberger-Treiman relation) and $h_{A}=2.03$ (smaller but not too far off the large- $\mathcal{N}_{c}$ value). However, the values for the $B_{i}$ 's came out different than those found later in $\pi N$ scattering: using Eq. (54), $c_{1}=2.2 \mathrm{GeV}^{-1}, c_{3}=-0.7 \mathrm{GeV}^{-1}$, and $c_{4}=2.7$ $\mathrm{GeV}^{-1}$, while from the leading delta contribution alone (because $B_{4}$ appears here only in higher order) $c_{2} \sim 1.8 \mathrm{GeV}^{-1}$. These values are to be compared to those in Table [; one concludes that $B_{3}$ is too big and of wrong sign, while $B_{2}$ is too small and $B_{1}$ too big, and/or the delta contributions are too small. For a cutoff of $\Lambda=780 \mathrm{MeV}$, the coefficients $C_{2 n}$ of 
$1 \mathrm{SO}$

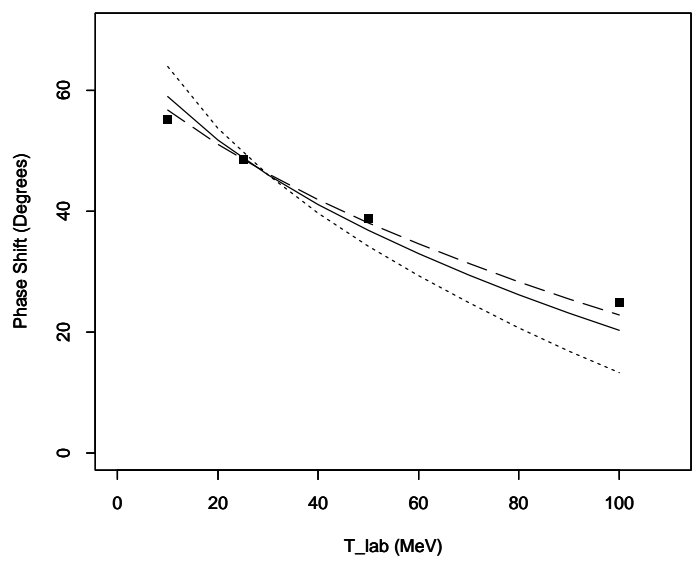

3D1

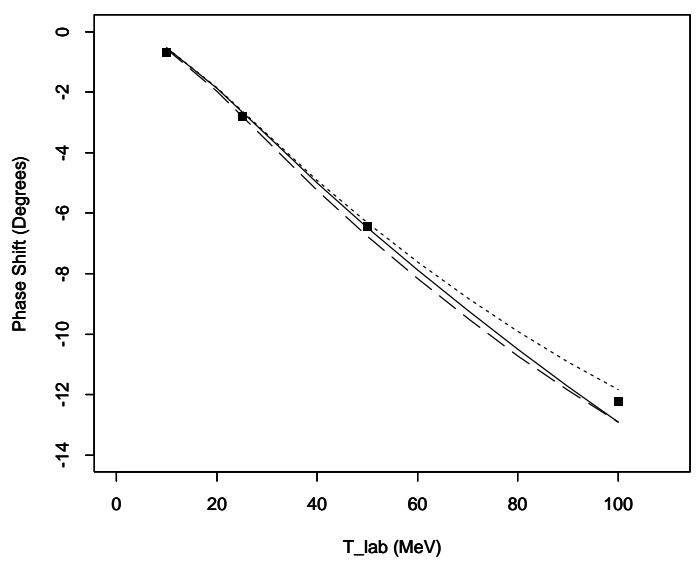

$3 \mathrm{~S} 1$

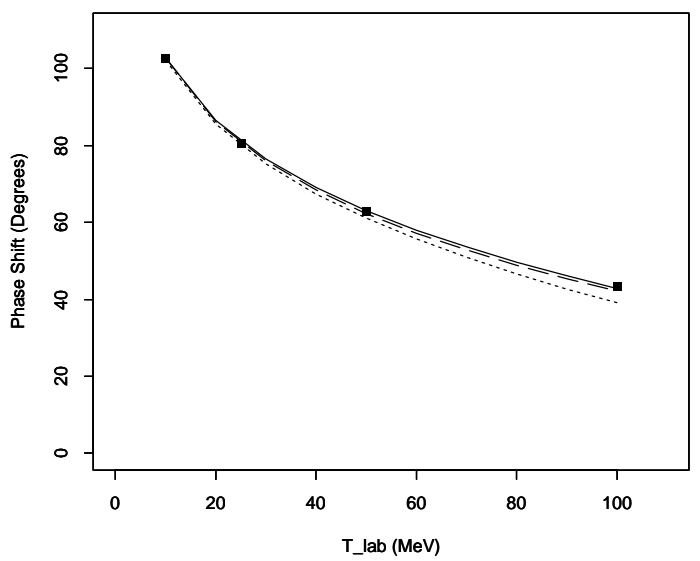

E1

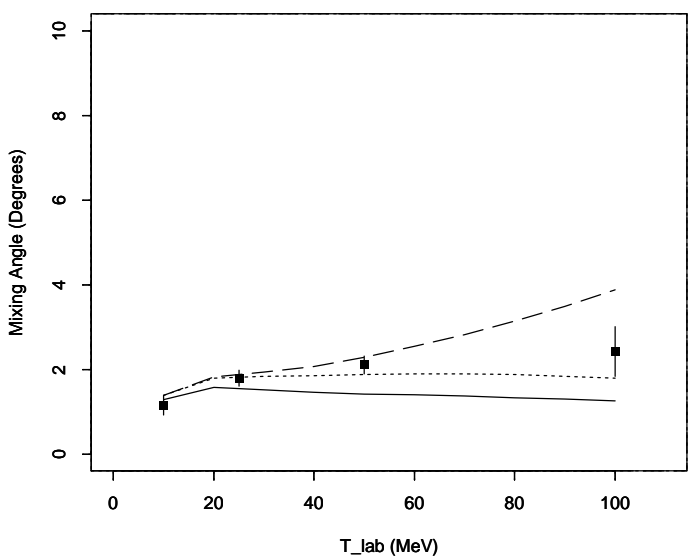

FIG. 10. ${ }^{1} S_{0},{ }^{3} S_{1}$, and ${ }^{3} D_{1} N N$ phase shifts and $\varepsilon_{1}$ mixing angle in degrees as functions of the laboratory energy in MeV: EFT up to $\nu=3$ for cutoffs of 500 (dotted), 780 (dashed), and 1000 $\mathrm{MeV}$ (solid line); and Nijmegen PSA (squares).

the contact interactions were found to scale approximately as in Eq. (110) with $M_{Q C D} \sim 500$ $\mathrm{MeV}$ for $M_{n u c}=F_{\pi}$.

A number of papers have subsequently examined different aspects of this EFT. In Ref. |105 it was pointed out that the parameters $A_{i}$ in Eq. (111) needed not be included explicitly in the fit, as one combination is fixed as a numerically small relativistic correction 106, while the $A_{1}$ contribution can be absorbed in the $C_{2}^{(6)}$ of Eq. (92) and in the $\pi N N$ coupling. Note that the above EFT potential is energy dependent. As pointed out in Sect. IID, equivalent potentials can be obtained through unitary transformations. An energy-independent potential is more convenient in many situations, and the corresponding version was derived in Refs. 107,105, 106. The effect of the unitary transformation is essentially to remove the third term in Eq. (111) and at the same time to modify the TPE potential. It corresponds in what box diagrams are concerned to a change from the Brueckener and Watson [102 to the TMO 108 potential. The latter has a further advantage: it displays explicitly a 
TABLE IV. Results from EFT fits at $\nu=3$ for various cut-offs $\Lambda$ and experimental values for the deuteron binding energy $(B)$, magnetic dipole moment $\left(\mu_{d}\right)$, electric quadrupole moment $\left(Q_{E}\right)$, asymptotic $D / S$ ratio $(\eta)$, and $D$-state probability $\left(P_{D}\right)$.

\begin{tabular}{ccccc}
\hline \hline Deuteron & \multicolumn{3}{c}{$\Lambda(\mathrm{MeV})$} & \\
\cline { 2 - 4 } quantities & 500 & 780 & 1000 & Experiment \\
\hline$B(\mathrm{MeV})$ & 2.15 & 2.24 & 2.18 & $2.224579(9)$ \\
$\mu_{d}\left(\mu_{N}\right)$ & 0.863 & 0.863 & 0.866 & $0.857406(1)$ \\
$Q_{E}\left(\mathrm{fm}^{2}\right)$ & 0.246 & 0.249 & 0.237 & $0.2859(3)$ \\
$\eta$ & 0.0229 & 0.0244 & 0.0230 & $0.0271(4)$ \\
$P_{D}(\%)$ & 2.98 & 2.86 & 2.40 & \\
\hline \hline
\end{tabular}

cancellation of the isoscalar spin-independent central and of the isovector spin-dependent interactions, which with a energy-dependent choice comes only through a combination of terms in the potential and its iterations. The components of the EFT TPE potential were studied in more detail in Ref. 93. In particular, it is shown explicitly that (i) relativistic corrections are mostly small (although in the weaker components they can make relatively large contributions); (ii) both isoscalar central and spin-orbit potentials are numerically similar to $\sigma$ and $\omega$ exchange in models, if the $B_{i}$ 's take values given by $\pi N$ scattering; (iii) the OPE isovector tensor potential is reduced by the TPE contribution; (iv) a subset - which is invariant under pion field redefinition - of the $\nu=\nu_{\min }+4$, two-loop TPE diagrams is small and repulsive. (Other modern studies of chiral aspects of TPE include Ref. [109.)

The fair agreement of this first calculation and data up to center-of-mass momenta $Q \sim 300 \mathrm{MeV}$ suggests that this may eventually become an alternative to other, more model-dependent approaches to the two-nucleon problem. In particular, it hints that a $\sigma$ exchange might be unnecessary. The relative cutoff insensibility also might be indication that renormalization is being performed correctly. The large values of the cutoff and the reasonable value of the contact terms are auspicious for the range of validity of the theory. Note that to this order this EFT includes all interactions incorporated to date in the perturbative-pion EFT and then some. More detailed analyses of the effects of different components of this potential on the fit have appeared since. In Ref. [70 the same EFT potential (with the same type of cutoff) as in Refs. 12, 15, 16] but without TPE (and energydependence in OPE) was refitted to the Nijmegen PSA [78 in three channels, ${ }^{1} S_{0},{ }^{1} P_{1}$, and ${ }^{3} D_{2}$, below center-of-mass energy of $100 \mathrm{MeV}$. An error analysis shows explicitly how the second-order corrections improve over the leading-order fits, particularly if in a global fit weights are assigned to data. It is confirmed that pion effects contribute little to the ${ }^{1} S_{0}$ phase shift in this energy range (as expected from fine-tuning), but play a more important role in higher-wave phases (as expected by power counting). The error analysis hints at a breakdown of the expansion at $M \sim 300 \mathrm{MeV}$, and shows that cutoff effects are minimal when $\Lambda \sim M$. It is suggested that the breakdown at $M \sim 300 \mathrm{MeV}$ is related to the neglect of TPE. Similar conclusions were reached in Ref. [95]. More recently, (the tail of the energy-independent version of) the EFT TPE potential in the limit of a heavy delta was substituted for (the tail of) the one-boson exchange in a Nijmegen phase-shift reanalysis 
of $p p$ data below $350 \mathrm{MeV}$ 110. A drop in $\chi^{2}$ was observed. $c_{1}$ was found to be poorly determined by the fit; fixing it from the sigma-term determined by the modern $\pi N$ PSA of Ref. [111], the best-fit values are

$$
c_{1}=-0.76(7) \mathrm{GeV}^{-1}, \quad c_{3}=-5.08(28) \mathrm{GeV}^{-1}, \quad c_{4}=4.70(70) \mathrm{GeV}^{-1} .
$$

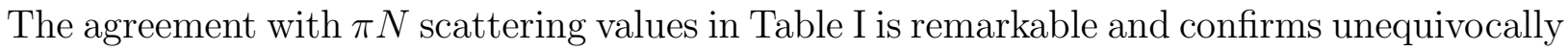
the validity of chiral TPE. Taken together, these new results suggest that a more extensive search of parameters might improve the quality of the EFT potential fit.

Isospin violation. We can order isospin-breaking effects in the potential in the same way done in Sect. III [15,18]. At $Q \sim M_{n u c} \gg \aleph$, photon exchanges are indeed perturbative, as discussed in Sect. IVA. Here I will concentrate on effects beyond those considered there, that is, on those stemming from the quark masses, from indirect electromagnetic effects, and from simultaneous pion-photon exchange. I follow the standard nomenclature and refer to an isospin-symmetric potential as "class I", to a potential that breaks charge dependence but not charge symmetry — defined as a rotation of $\pi$ around the 2-axis in isospin spaceas "class II", to one that breaks charge symmetry but vanishes in the $n p$ system as "class III", and to one that breaks charge symmetry but is present in the $n p$ system as "class IV".

No isospin-violating effects appear at leading order, $\nu=\nu_{\min }$, so the leading potential is class I. The first effect appears at $\nu=\nu_{\text {min }}+1$ in the form of a class II isospin violation from pion mass splitting $\left(\Delta m_{\pi}^{2} \propto \alpha M_{Q C D}^{2}\right)$ in OPE and from Coulomb photon exchange. One order down, $\nu=\nu_{\text {min }}+2$, a class III force appears mainly from the quark mass difference through breaking in the $\pi N N$ coupling $\left(\beta_{1}=O\left(\varepsilon m_{\pi}^{2} / M_{Q C D}^{2}\right)\right)$ in OPE, from contact terms $\left(\gamma_{s, \sigma}=O\left(\varepsilon m_{\pi}^{2} / M_{Q C D}^{4}\right)\right)$, and from the nucleon mass difference $\left(\Delta m_{N}=O\left(\varepsilon m_{\pi}^{2} / M_{Q C D}\right)\right)$. Therefore, to order $\nu=\nu_{\text {min }}+2$ the isospin-violating nuclear potential is a two-nucleon potential of the form

$$
V=V_{\mathrm{II}}\left[\left(t_{1}\right)_{3}\left(t_{2}\right)_{3}-\boldsymbol{t}_{1} \cdot \boldsymbol{t}_{2}\right]+V_{\mathrm{III}}\left[\left(t_{1}\right)_{3}+\left(t_{2}\right)_{3}\right]
$$

where

$$
\begin{gathered}
V_{\mathrm{II}}=-\left(\frac{2 g_{A}}{F_{\pi}}\right)^{2} \frac{\vec{q} \cdot \vec{\sigma}_{1} \vec{q} \cdot \vec{\sigma}_{2}}{\left(\vec{q}^{2}+m_{\pi^{0}}^{2}\right)\left(\vec{q}^{2}+m_{\pi^{ \pm}}^{2}\right)}\left(\Delta m_{\pi}^{2}+\Delta m_{N}^{2}\right), \\
V_{\mathrm{III}}=\frac{2 g_{A} \beta_{1}}{F_{\pi}^{2}} \frac{\vec{q} \cdot \vec{\sigma}_{1} \vec{q} \cdot \vec{\sigma}_{2}}{\vec{q}^{2}+m_{\pi}^{2}}-\left(\gamma_{s}+\gamma_{\sigma} \vec{\sigma}_{1} \cdot \vec{\sigma}_{2}\right) .
\end{gathered}
$$

Finally, class IV forces appear only at order $\nu=\nu_{\text {min }}+3$. One concludes that the symmetries of QCD naturally suggest a hierarchy of classes in the nuclear potential [15, 18]:

$$
\left\langle V_{\mathrm{M}+1}\right\rangle /\left\langle V_{\mathrm{M}}\right\rangle \sim O\left(Q / M_{Q C D}\right)
$$

where $\left\langle V_{\mathrm{M}}\right\rangle$ denotes the average contribution of the leading class $\mathrm{M}$ potential. This qualitatively explains, for example, the observed isospin structure of the Coulomb-corrected scattering lengths, $a_{n p} \simeq 4 \times\left(\left(a_{n n}+a_{p p}\right) / 2-a_{n p}\right) \simeq 4^{2} \times\left(a_{p p}-a_{n n}\right)$. 


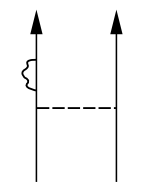

(a)

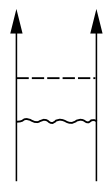

(e)

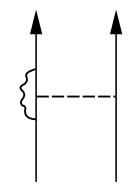

(b)

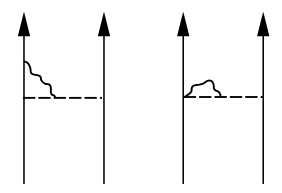

(c)

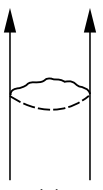

(g) (d)

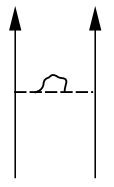

(h)

FIG. 11. Electromagnetic loops contributing to isospin violation in the one-pion-range nuclear force in first non-vanishing order. Solid lines are nucleons, dashed lines are pions, and wavy lines are photons. Other orderings are also included, but iteration of static OPE and Coulomb exchange is excluded from (e).

One can use the above formalism to do consistent and systematic calculations of isospin violation. The isospin-violating potential of range $\sim 1 / m_{\pi}$ up to $\nu=\nu_{\min }+3$ was computed in Refs. [112, [13]. It consists of diagrams where one pion flies between the two nucleons, constructed out of tree-level diagrams with interactions of index up to 3, plus 16 (not counting trivial particle permutations) non-vanishing one-loop diagrams, comprising pion and nucleon self-energy corrections, pion-nucleon vertex corrections, and simultaneous pion-photon exchange - see Fig. 11. Most of these diagrams are ultraviolet divergent; dimensional regularization was used and $1 /(D-4)$ terms cancelled against bare coefficients. All diagrams with photons are also individually infrared divergent; a photon mass is introduced and removed at the end, the sum being infrared finite. The result is invariant under both gauge transformation and pion-field redefinition:

$$
V_{\pi \gamma}(\vec{q})=\frac{2 \alpha g_{A}^{2}}{\pi F_{\pi}^{2}}\left(\boldsymbol{t}_{1} \cdot \boldsymbol{t}_{2}-\left(t_{1}\right)_{3}\left(t_{2}\right)_{3}\right) \frac{\vec{\sigma}_{1} \cdot \vec{q} \vec{\sigma}_{2} \cdot \vec{q}}{q^{2}}\left[\frac{\left(1-q^{2} / m_{\pi}^{2}\right)^{2}}{\left(1+q^{2} / m_{\pi}^{2}\right) q^{2} / m_{\pi}^{2}} \ln \left(1+q^{2} / m_{\pi}^{2}\right)-1\right] .
$$

Its isospin structure allows only charged-pion exchange and therefore affects only $n p$ scattering. This $\pi \gamma$ potential has been incorporated in a Nijmegen phase shift reanalysis of $n p$ data below $350 \mathrm{MeV}$ [112]. We can use the values for the $\pi N N$ coupling constants determined by the analysis to find that their isospin breaking is consistent with zero, with an uncertainty comparable to our expectation from dimensional analysis and from $\pi-\eta-\eta^{\prime}$ mixing [114. Similarly, $\gamma_{s}$ might be viewed as originating in $\rho-\omega$ mixing, while pseudovector-meson exchange (in particular close-lying doublets such as $a_{1}-f_{1}$ ) exchange contribute to the $\gamma_{\sigma}$ spin-spin force [114,115.

\section{FEW-NUCLEON SYSTEMS}

Few-body systems provide non-trivial testground for ideas developed in two-body dynamics. As we have seen, the deuteron is sufficiently dilute that an EFT for $Q \sim \aleph$ can 
be used to describe it well. Such an EFT is essentially equivalent to an effective range expansion. The latter has been successfully applied to reactions involving the deuteron and photons, but difficulties have been encountered in the three-body system. In Sect. $\nabla A$ we will see how the EFT can be extended to the three-body system. In this case some fairly unusual renormalization takes place, which makes the EFT method much more challenging and thus interesting than in its more standard perturbative applications. The EFT for higher momenta where pions are treated perturbatively shares the same leading order as the pionless EFT, differing only in that pion exchange appears explicitly together with a contact correction in subleading order. The important question to be answered is whether few-nucleon bound states can be dealt with in the approximation where leading-order interactions are taken as short ranged. In Sect. $\mathrm{VB}$ still higher momenta are considered, and the connection to potential model approaches discussed. We can use this EFT to organize the various few-body-force structures.

\section{A. Very low and low energies}

Symmetries do not forbid three-nucleon contact interactions. In order to extend the EFT to the three-nucleon system we need to find the size of three-nucleon forces relative to two-nucleon forces. As in Sect. IVA, I omit at first spin and isospin variables. I also neglect electromagnetic interactions. Requiring invariance under small-velocity Lorentz, parity, and time-reversal transformations, at sufficiently low momenta, the Lagrangian involving six fields $\psi$ is

$$
\mathcal{L}=-\frac{D_{0}}{6}\left(\psi^{\dagger} \psi\right)^{3}+\ldots,
$$

where "..." stand for terms with more derivatives, which are suppressed at low momenta. Note that an $S$-wave contact three-body interaction is allowed for bosons. The same is true in the case of three nucleons in a spin $S=1 / 2$ state, but the Pauli principle forbids an $S$-wave interaction for three nucleons in a $S=3 / 2$ state. The first possible three-body interaction in the latter channel contains an extra $Q^{2}$, and should be relatively less important.

A comparison between the tree-level connected three-body diagrams allows us to guess the relative size of two- and three-body interactions. We saw in Sect. IVA that operators involving four nucleon fields contain the small scale $\aleph$. At momenta $Q \sim \aleph$, two sequential two-body $C_{0}$ interactions contribute $O\left((4 \pi / m \aleph)^{2} m / \aleph^{2}\right)$. If we assume that the coefficients $D_{2 n}$ of the terms with $2 n$ derivatives in Eq. (120) are of natural size, $D_{2 n} \sim(4 \pi)^{2} / m M^{4+2 n}$, then we expect three-body-force effects to be of relative $O\left((\aleph / M)^{4+2 n}\right)$. But does the small scale $\aleph$ not contaminate operators involving six nucleon fields? This question is intimately related to the renormalization group flow of three-body interactions with the mass scale introduced in the regularization procedure. This flow, in turn, depends on the behavior of the sum of two-body contact contributions to three-body amplitude as function of the renormalization scale, or equivalently, as function of the ultraviolet cutoff $\Lambda$.

It is convenient to rewrite this theory by introducing the dimeron field $T$ of Sect. IVA It is straightforward to show - for example, by a Gaussian path integration - that the Lagrangian (120) is equivalent to Eq. (72) supplemented with 


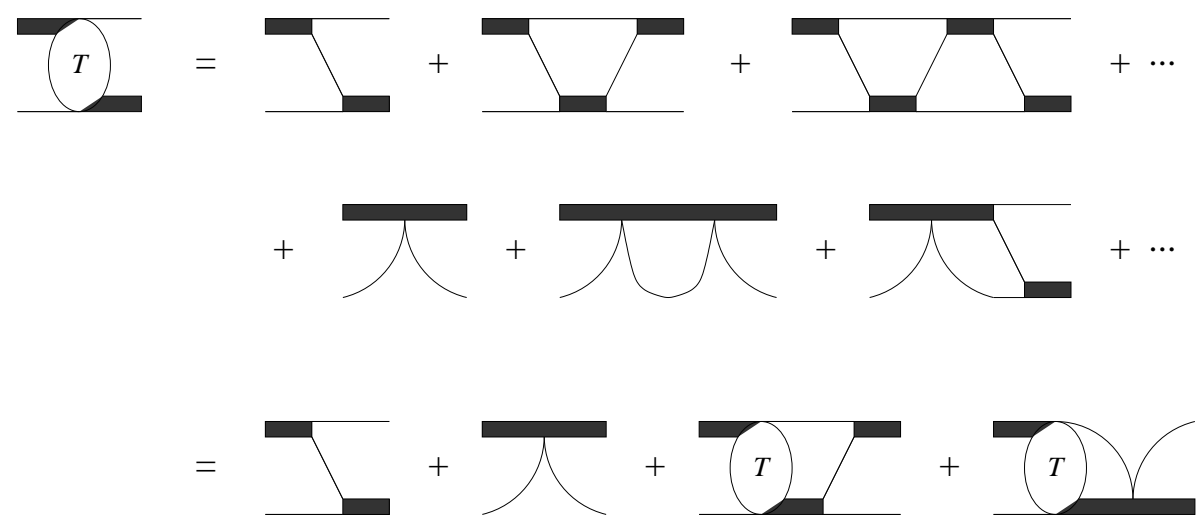

FIG. 12. The amplitude $T$ for particle/bound-state scattering as a sum of dressed pinball and three-body-force diagrams (first and second lines) and as an integral equation (third line).

$$
\mathcal{L}=h T^{\dagger} T \psi^{\dagger} \psi+\ldots
$$

In leading order, since the kinetic term of the dimeron can be neglected, observables will depend on the parameter appearing explicitly in Eq. (121) only through the combination $-3 h g^{2} / \Delta^{2} \equiv D_{0}$.

Let us consider elastic particle/bound-state scattering at $Q \sim \aleph$ 116 118]. (Threebody bound states manifest themselves as poles at negative energy. Both the inelastic channel and three-particle scattering involve the same type of diagrams considered here and can be obtained by a straightforward extension of the following arguments.) Diagrams contributing to the amplitude are depicted in Fig. 12. We have already seen that at these momenta all terms in the full dimeron propagator of Fig. 1 are equally important. It is easy to see that an $L$-loop diagram involving only two-body interactions ("pinball" diagram) is $O\left(\left(m g^{2} / Q^{2}\right) \times\left(m g^{2} Q / 4 \pi\left(\Delta+m g^{2} Q / 4 \pi\right)\right)^{L}\right)$. Pinball diagrams are thus (superficially) ultraviolet finite, and are all of the same order for $m g^{2} Q / 4 \pi \Delta=Q / \aleph \sim 1$. The threebody amplitude is then the solution of an integral equation. This equation, including the three-body force, is also depicted in Fig. 12.

I choose the following kinematics: the incoming particle and bound state are on-shell with four-momenta $\left(k^{2} / 2 m,-\vec{k}\right)$ and $\left(k^{2} / 4 m-B_{2}, \vec{k}\right)$, respectively. The outgoing particle and bound state are off-shell with four-momenta $\left(k^{2} / 2 m-\varepsilon,-\vec{p}\right)$ and $\left(k^{2} / 4 m-B_{2}+\varepsilon, \vec{p}\right)$; the on-shell point has $\varepsilon=k^{2} / 2 m-p^{2} / 2 m$ and $p=k$. The total energy is $E=3 k^{2} / 4 m-B_{2}$. At very low momenta, we can limit ourselves to the more important $S$-wave. Using the boosted dimeron propagator (74), after performing the integration over the fourth component of the loop momentum, setting $\epsilon=k^{2} / 2 m-p^{2} / 2 m$, and projecting onto the $S$-wave, we find that the amplitude $a(k, p)$ normalized so that $a(0,0)=-a_{3}$ is the particle/bound-state scattering length satisfies 118

$$
a(k, p)=M(k, p ; k)+\frac{2 \lambda}{\pi} \int_{0}^{\Lambda} d q M(q, p ; k) \frac{q^{2}}{q^{2}-k^{2}-i \epsilon} a(k, q)
$$

with the kernel 


$$
M(q, p ; k)=\frac{4}{3}\left(\frac{1}{a_{2}}+\sqrt{\frac{3 p^{2}}{4}-m E}\right)\left[\frac{1}{p q} \ln \left(\frac{q^{2}+q p+p^{2}-m E}{q^{2}-q p+p^{2}-m E}\right)+\frac{h}{m g^{2}}\right] .
$$

Here $\lambda=1$ for bosons. The same equation is valid in the case of fermions, with different values of $\lambda$. For three nucleons in a spin $S=3 / 2$ state, this equation holds with $\lambda=-1 / 2$ and $h=0$. For three nucleons in a spin $S=1 / 2$ state a pair of coupled integral equations applies, with properties similar to the bosonic case. Eqs. (122) and (123) reduce to the expressions found in Refs. [119,116, 117] when $h=0$. Note that the perturbative series shown in Fig. 12 corresponds to a perturbative solution of the integral equation for small $\lambda$. The solution of Eq. (122) is complex even below the threshold for the breakup of the two-particle bound state due to the $i \epsilon$ prescription. To facilitate the discussion one uses the function $K(k, p)$ that satisfies the same Eq. (122) as $a(k, p)$ but with the $i \epsilon$ substituted by a principal value prescription. $K(k, p)$ is real below the breakup threshold. $a(k, k)$ and, consequently, the scattering matrix can be obtained from $K(k, p)$ through

$$
a(k, k)=\frac{K(k, k)}{1-i k K(k, k)} .
$$

In order to understand the renormalization group flow due to two-body forces, let us first take $h=0$. When $p \gg 1 / a_{2}$ (but $\left.k \sim 1 / a_{2}\right)$, the inhomogeneous term is small $\left(O\left(1 / p a_{2}\right)\right)$, the main contribution to the integral comes from the region $q \sim p$, and the amplitude satisfies the approximate equation

$$
K(k, p)=\frac{4 \lambda}{\sqrt{3} \pi} \int_{0}^{\Lambda} \frac{d q}{q} \ln \left(\frac{q^{2}+p q+p^{2}}{q^{2}-p q+p^{2}}\right) K(k, q) .
$$

Now, in the limit $\Lambda \rightarrow \infty$ there is no scale left. Scale invariance suggests solutions of the form $K(k, p)=p^{s}$, which exist only if $s$ satisfies

$$
1-\frac{8 \lambda}{\sqrt{3} s} \frac{\sin \frac{\pi s}{6}}{\cos \frac{\pi s}{2}}=0
$$

If $K(k, p)$ is a solution, $K\left(k, p_{\star}^{2} / p\right)$ is also a solution for arbitrary $p_{\star}$. Because of this special conformal symmetry, the solutions of Eq. (126) come in pairs.

For $\lambda<\lambda_{c}=3 \sqrt{3} / 4 \pi \simeq 0.4135$, Eq. (126) has only real roots. For $\lambda=-1 / 2$, there are solutions $s= \pm 2, \pm 2.17, \ldots$ In this case an acceptable solution of Eq. (125) decreases in the ultraviolet,

$$
K\left(k, p \gg 1 / a_{2}\right)=C p^{-|s|} .
$$

For finite cutoff, the solution should still have this form as long as $p \ll \Lambda$. The overall constant $C(\Lambda)$ cannot be fixed from the homogeneous asymptotic equation, but is determined by matching the asymptotic solution onto the solution at $p \sim 1 / a_{2}$ of the full Eq. (122). Because of the fast ultraviolet convergence, the full solution to Eq. (122) is expected to be insensitive to the choice of regulator, so that a well-defined $\lim _{\Lambda \rightarrow \infty} C(\Lambda)$ can be found. There is thus no evidence for a contamination of three-body forces by the small scale $\aleph$ in the $S=3 / 2$ channel of $S$-wave nucleon-deuteron $(N d)$ scattering. Up to and including 


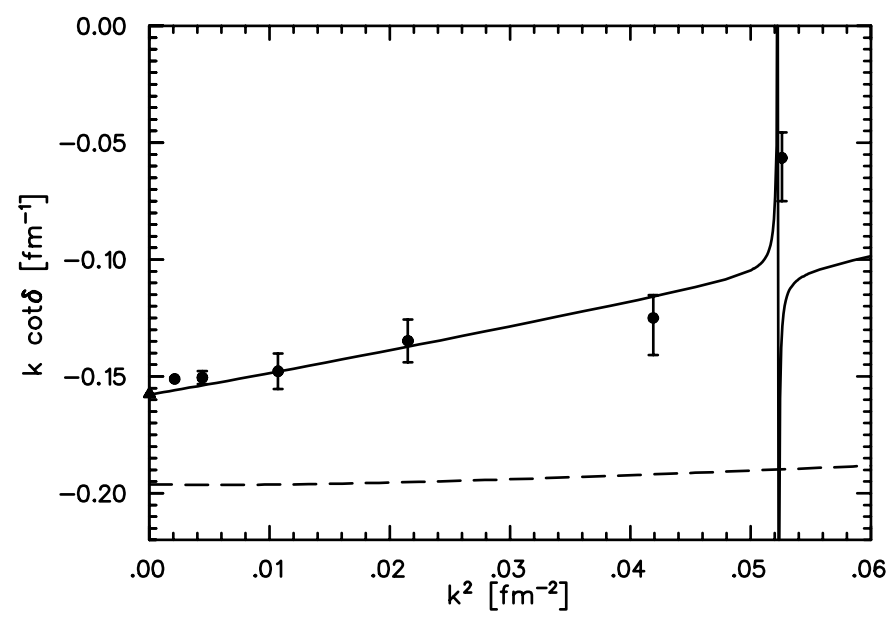

FIG. 13. $k \cot \delta$ in $\mathrm{fm}^{-1}$ for $S$-wave $N d$ scattering in the $S=3 / 2$ channel as a function of $k^{2}$ in $\mathrm{fm}^{-2}$ in the EFT to $O(1)$ (dashed line) and $O\left(\left(\aleph / M_{n u c}\right)^{2}\right)$ (solid line). Circles are from the PSA in Ref. [120] and the triangle is from Ref. [121].

relative $O\left((Q / M)^{5}\right)$, only two-body forces need to be included. A calculation using Eq. (72) up to and including relative $O\left((Q / M)^{2}\right)$ was carried out in Refs. 116,117. As expected, cutoff dependence was found only for $p \sim \Lambda$. The result for the phase shifts for energies up to the break-up point is shown in Fig. 13: the result of the leading-order $(O(1))$ calculation [119] is given by the dashed line and the $O\left((\aleph / M)^{2}\right)$ result [117] by the solid line. These are compared to data points at finite energy from a PSA of $N d$ scattering data [120], and to the much more precise (nearly) zero-energy point [121]. We expect errors in our calculation to be of $\left.O\left(\aleph / M_{n u c}\right)^{3}\right)$. At zero energy, we find $a_{3}^{(3 / 2)}=6.33 \pm 0.10 \mathrm{fm}$ [116, compared to the experimental value $a_{3}^{(3 / 2)}=6.35 \pm 0.02 \mathrm{fm}[121]$. This is an extremely accurate lowenergy theorem! Our results seem to deviate from a simple effective range type expansion only around the pole at $\sim 0.05 \mathrm{fm}^{-2}$. (A pole in $k \cot \delta$ corresponds to a zero in the scattering matrix, which does not carry special meaning.) This pole does not appear in potential model calculations, and presumably will be (re)moved by higher-order terms that we have not yet included. The calculation of higher-order corrections involves the knowledge of further counterterms like the ones giving rise to $S-D$ mixing. These parameters can be determined either by fitting $N N$ data or by matching with another effective theory involving explicit pions - valid up to higher energies. If more precise experimental data - particularly at zero-energy - appear, we would be facing a unique situation where highprecision calculations in strong-interaction physics can be carried out systematically and tested.

For $\lambda=1$, on the other hand, there are purely imaginary solutions: $s= \pm i s_{0}$, where $s_{0} \simeq 1.0064$. The solution of Eq. (125) for $p \ll \Lambda$ is

$$
K\left(k, p \gg 1 / a_{2}\right)=C \cos \left(s_{0} \ln \frac{p}{\Lambda_{\star}}\right),
$$

where, on dimensional grounds, $\Lambda_{\star}(\Lambda) \propto \Lambda$. This solution has two constants $C(\Lambda)$ and 
$\Lambda_{\star}(\Lambda)$, and implies that the solution of Eq. (122) is not unique in the limit $\Lambda \rightarrow \infty$ [122]. The undetermined phase $\Lambda_{\star}$ arises from special conformal symmetry, as there is no way here to select a preferred oscillatory solution. Solutions of Eq. (122) for $\lambda=1$ and finite $\Lambda$ (but $h=0$ ) were obtained in Ref. 118. We have verified that in the region $1 / a_{2} \ll p \ll \Lambda$ the solutions indeed have the form (128), with $\Lambda_{\star}$ approximately linear in the cutoff. The amplitude $C$ was found to be approximately proportional to $\cos \left(s_{0} \ln \left(\Lambda_{\star} a_{2}\right)\right)$. This dependence generates some points where the amplitude is cutoff independent, but apart from them, the amplitude does not show signs of converging as $\Lambda \rightarrow \infty$. Since the solution for small $p$ has to match onto the large- $p$ solution, the cutoff dependence leaks into the low-momentum region. Small differences in the asymptotic phase lead to large differences in, for example, the particle/bound-state scattering length. This leakage of highmomentum behavior into the low-momentum physics is indication that we are not performing renormalization consistently with our expansion. Note that if one were to truncate the series of diagrams in Fig. 12 at some finite number of loops one would miss the asymptotic behavior of $K(k, p)$ that generates this cutoff dependence. This is because $s_{0}$ (and its expansion in powers of $\lambda$ ) vanish in a neighborhood of $\lambda=0$. The truncation of the series in Fig. 12 is equivalent to perturbation theory in $\lambda$, and cannot produce a non-vanishing $s_{0}$. We are here facing truly non-perturbative aspects of renormalization, which resemble a phase transition.

Faced with regulator dependence, one needs to modify the leading-order calculation, that is, to change the bookkeeping of the higher-energy behavior of the theory through the addition of at least one new interaction. In an EFT, one adds local counterterms. Here the only alternative is to introduce a three-body force, but the present case is complicated by the fact that the cutoff dependence of the amplitude is non-analytic around $p=0$. This, however, does not mean that the renormalization program in this low-energy EFT is doomed: a three-body force term of sufficient strength contributes not only at tree level, but also in loops dressed by any number of two-particle interactions. It is convenient to rewrite the three-body force as

$$
h(\Lambda)=\frac{2 m g^{2} H(\Lambda)}{\Lambda^{2}} .
$$

$H(\Lambda)$ has to be at least big enough to give a non-negligible contribution in the $p \sim k \sim$ $\Lambda$ region. This means that the dimensionless quantity $H(\Lambda)$ has to be at least of $O(1)$. Assuming minimal strength, the three-body force has the feature that its contribution to the inhomogeneous term is small compared to the contribution from the two-body interaction, as it is at most $p / \Lambda$ of the latter. When $p \gg 1 / a_{2}$ (but $k \sim 1 / a_{2}$ ), the amplitude satisfies a new approximate equation, with a three-body interaction added to the rhs of Eq. (125). The solution $K(k, p \sim \Lambda)$ has a complicated form. But, because in the range $1 / a_{2} \ll p \ll \Lambda$ the three-body force term is suppressed by $p / \Lambda$ compared to the logarithm, the behavior (128) is still correct in the intermediate region. Now the phase is function of both $\Lambda$ and $H(\Lambda)$, so if $H(\Lambda)$ is chosen appropriately, we can make $\Lambda_{\star}$ cutoff independent. Matching with the $p \sim 1 / a_{2}$ solution should then determine the scattering length $a_{3}=a_{3}\left(\Lambda_{\star}\right)$ and the low-energy dependence of the amplitude. For $p \sim 1 / a_{2}$, Eq. (122) becomes

$$
\frac{3}{4} \frac{K(k, p)}{\left(\frac{1}{a_{2}}+\sqrt{\frac{3 p^{2}}{4}-m E}\right)}=\frac{1}{p k} \ln \left(\frac{p^{2}+p k+k^{2}-M E}{p^{2}-p k+k^{2}-M E}\right)
$$




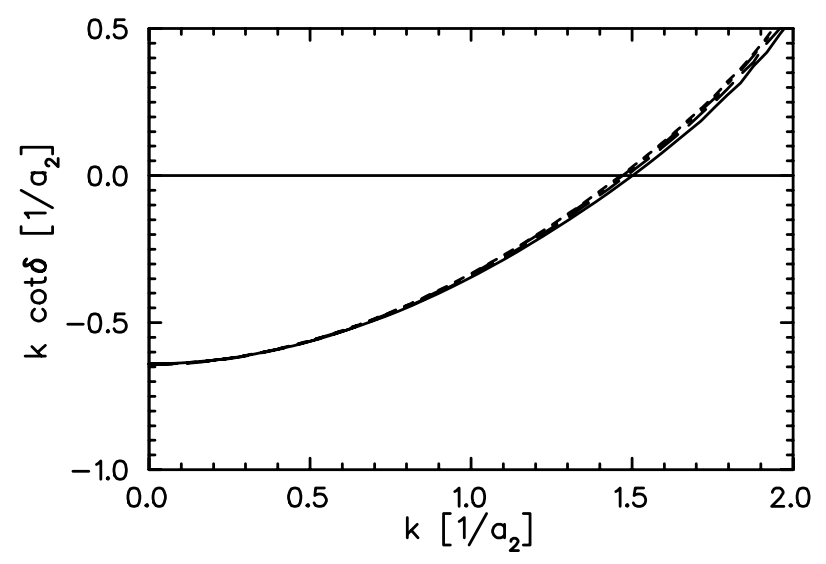

FIG. 14. $k \cot \delta$ in units of $1 / a_{2}$ for $S$-wave particle/bound-state scattering in the $S=0$ case as a function of $k$ in units of $1 / a_{2}$ in the EFT to leading order, for $\Lambda_{\star}=19.5 a_{2}^{-1}$ and different cutoffs $\left(\Lambda=42.6,100.0,230.0,959.0 \times a_{2}^{-1}\right)$.

$$
\begin{aligned}
& +\frac{2 \lambda}{\pi p} \int_{0}^{\mu} d q \ln \left(\frac{p^{2}+p q+q^{2}-M E}{p^{2}-p q+q^{2}-M E}\right) \frac{q K(k, q)}{q^{2}-k^{2}} \\
& +\frac{4 \lambda}{\pi} \int_{\mu}^{\Lambda} d q\left[\frac{1}{q^{2}}+\frac{H(\Lambda)}{\Lambda^{2}}\right] K(k, q),
\end{aligned}
$$

where $\mu$ is an arbitrary scale such that $\mu \ll \Lambda$, and we have dropped terms which are smaller by powers of $p / \Lambda$ or $\mu / \Lambda$. One can use this equation to obtain an approximate expression for $H=H(\Lambda)$. By integrating out modes between $\Lambda^{\prime}>\Lambda$ and $\Lambda$ and demanding that the low-energy amplitude be unchanged, we find [118

$$
H(\Lambda)=-\frac{\sin \left(s_{0} \ln \left(\frac{\Lambda}{\Lambda_{\star}}\right)-\operatorname{arctg}\left(\frac{1}{s_{0}}\right)\right)}{\sin \left(s_{0} \ln \left(\frac{\Lambda}{\Lambda_{\star}}\right)+\operatorname{arctg}\left(\frac{1}{s_{0}}\right)\right)} .
$$

The last diagram in Fig. 12 can absorb the high-momentum modes of the diagram that precedes it.

The full solution of Eq. (122) now depends, apart from two-body parameters, on the new, physical parameter $\Lambda_{\star}$ (but not on the regulator). This equation was solved numerically with a non-vanishing $H(\Lambda)$ for several cutoffs. The three-body force that is necessary to keep $a_{3}$ unchanged was found in good accord with the analytical approximation (131). In Fig. 14 we see the results for the corresponding $K(k, k)^{-1}=k \cot \delta$, where $\delta$ is the $S$-wave phase shift for particle/bound-state scattering, for different cutoffs in the case $a_{3}=1.56 a_{2}$. The effective range, for example, is predicted as $r_{3}=0.57 a_{2}$.

We can extend the preceding analysis to the three-body bound-state problem. Since the inhomogeneous term did not play an important role in the above ultraviolet arguments, the latter hold for any bound state with binding energy $B_{3}$ comparable with $1 / m a_{2}^{2}$. Such bound states are shallow, having a size $\sim 1 / \sqrt{m B_{3}} \sim a_{2}$, and thus should be within range of the EFT. In principle, all bound states with size larger than $\sim r_{2}$ should be amenable to 


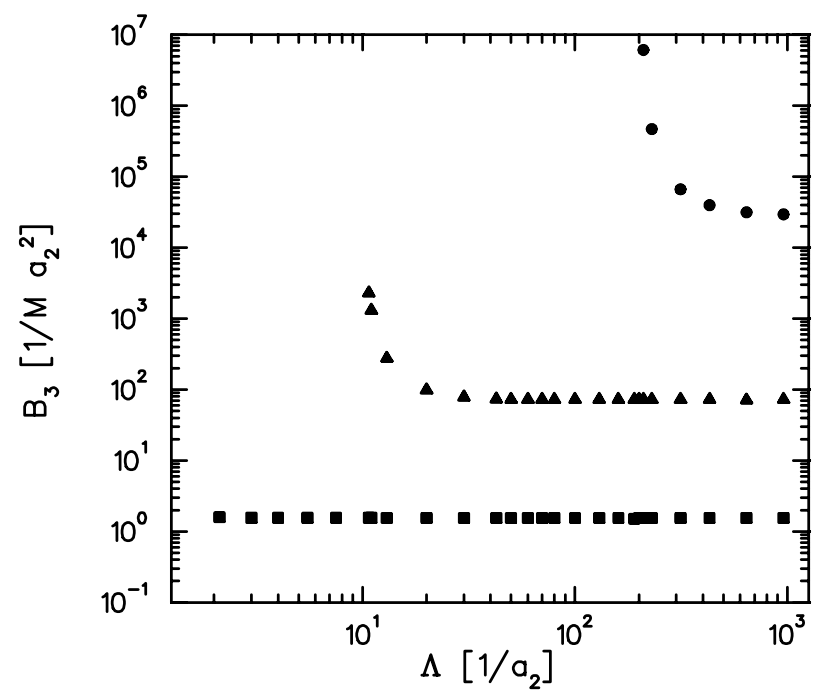

FIG. 15. Three-body binding energies $B_{3}$ in units of $1 / m a_{2}^{2}$ as functions of the cutoff $\Lambda$ in units of $1 / a_{2}$ in the EFT to leading order in the $S=0$ case, for $\Lambda_{\star}=19.5 a_{2}^{-1}$.

this EFT description. Their properties will be determined in first approximation by only $C_{0}$ and $\Lambda_{\star}$, while more precise information can be obtained in an expansion in $\aleph / M$. In Fig. 15 binding energies are shown for a range of cutoffs, with the three-body force adjusted to give a fixed scattering length $a_{3}=1.56 a_{2}$. (For particular values of $a_{3}$ and $\Lambda$, these results reduce to those in Ref. [123].) As we can see, for this value of $\Lambda_{\star}$ there exists a shallow bound state at $B_{3} \simeq 2 / m a_{2}^{2}$ whose binding energy is independent of the cutoff. This bound state has size $\sim 0.7 a_{2}$ and can thus be studied with the EFT. As we increase the cutoff, deeper bound states appear at $\Lambda_{n}=\Lambda_{0} \exp \left(n \pi / s_{0}\right)$ with $n$ an integer and $\Lambda_{0} \simeq 10$, so that for $\Lambda_{n-1} \leq \Lambda \leq \Lambda_{n}$ there are $n+1$ bound states. Similar results can be obtained for other values of $a_{3}$. By repeating this procedure we can in fact determine $a_{3}=a_{3}\left(\Lambda_{\star}\right)$ (or viceversa) and $B_{3}=B_{3}\left(\Lambda_{\star}\right)$ (or vice-versa). Eliminating $\Lambda_{\star}$, a universal curve $B_{3}=B_{3}\left(a_{3}\right)$ can be obtained. In the case of the shallowest bound state, this is seen in Fig. 16. This curve is known in the three-nucleon case as the Phillips line [124. It has been derived before in the context of models for the two-nucleon potential that differ in their high-momentum behavior. Varying among two-particle potential models one could expect to fill up the $B_{3} \times a_{3}$ plane. In the EFT, the high-momentum behavior of the two-particle potential is butchered, which causes no trouble in describing two-particle scattering at low-energies, but in the peculiar way described here, does require a three-body force. Varying among two-particle potential models is thus equivalent to varying the one parameter $\Lambda_{\star}$ of the three-body force. This spans a single curve $B_{3}=B_{3}\left(a_{3}\right)$ in the $B_{3} \times a_{3}$ plane. Our argument suggests that the Phillips line is a generic consequence of renormalization group invariance.

Corrections to this calculation come from operators with more derivatives, and they are discussed in Ref. [118. The first correction comes from $C_{2}$ in Eq. (57). It can be shown that the large $\Lambda$ dependence it introduces can be absorbed in $h$ itself, so that a finite, calculable contribution remains. Its effects are being calculated [125]. It is reasonable to assume then 


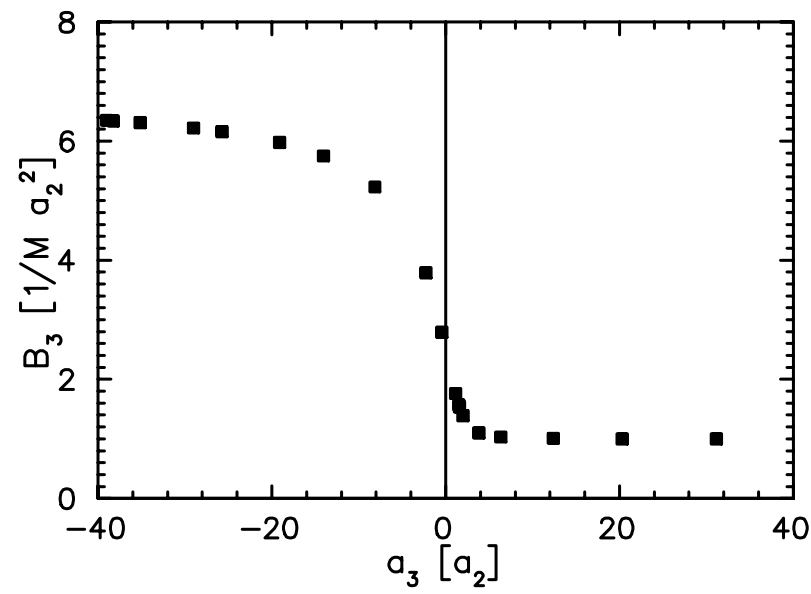

FIG. 16. Binding energy $B_{3}$ of the shallowest bound state in units of $1 / m a_{2}^{2}$ as function of the particle/bound-state scattering length $a_{3}$ in units of $a_{2}$ in the EFT to leading order in the $S=0$ case.

that more-derivative three-body forces do not get further enhanced by inverse powers of $\aleph$, so that

$$
D_{2(n+1)}^{\ldots(R)}=\frac{D_{2 n}^{\ldots(R)}}{M^{2}},
$$

In this case effects of three-body-force corrections could be accounted for perturbatively.

The above results hold for ${ }^{4} \mathrm{He}$ atoms 118 . In fact, it has recently been established that the two- ${ }^{4}$ He bound state ("dimer") is very shallow, with an average size $\langle r\rangle=62 \pm 10 \AA$ 126], more than an order of magnitude larger than the range of the interatomic potential. The lowenergy two- ${ }^{4} \mathrm{He}$ system should then be describable by contact two-body forces. In leading order, the measured size translates into a scattering length $a_{2}=124 \AA$, which determines the strength of the contact interaction. Unfortunately, although the three- ${ }^{4} \mathrm{He}$ ("trimer") has been observed [127], there seems to be no low-energy information on its properties nor on ${ }^{4} \mathrm{He} /$ dimer scattering. At least one three-body datum is needed to determine $\Lambda_{\star}$. Until such datum becomes available, we can only illustrate the method by using a phenomenological ${ }^{4} \mathrm{He}-{ }^{4} \mathrm{He}$ potential as a model of a microscopic theory. We select a potential [128] that is consistent with the recent measurement of the dimer binding energy. It gives for the twobody system $a_{2}=124.7 \AA$ and $r_{2} \simeq 7.4 \AA$. Three-body calculations are much more difficult to perform with such a phenomenological potential. An estimate for the ${ }^{4} \mathrm{He} /$ dimer scattering length is $a_{3}=195 \AA$. Ground and excited bound states have been reported; estimates for the shallowest bound state place it in the range $B_{3}=1.04-1.7 \mathrm{mK}$, while a deeper state lies around $B_{3}=0.082-0.1173 \mathrm{~K}$. There exists a prediction for the low-energy $S$-wave phase shift, albeit for a different potential, but $r_{3}$ could not be determined. Using such model we can estimate the range of validity of the EFT in momentum to be $\sim 1 / r_{2} \simeq 0.14 \AA^{-1}$, and the leading order to give an accuracy of $\sim r_{2} / a_{2} \simeq 0.06$, or about $10 \%$, at momenta $Q \sim 1 / a_{2}$. Using this potential's $a_{3} / a_{2}=1.56$ we can determine $\Lambda_{\star}$, and predict both the 
energy dependence in ${ }^{4} \mathrm{He} /$ dimer scattering and the trimer binding energy. In fact, in Fig. 14 I have used exactly this value of $a_{3}$, so it represents the lowest-order prediction for $k \cot \delta$ for atom/dimer scattering, from which we can extract an effective range $r_{3}=71 \AA$. From Fig. 15 we predict a bound state at $B_{3}=1.2 \mathrm{mK}$, which is certainly within the EFT. The next-to-shallowest bound state is small enough to be at the border of EFT applicability. For a sufficiently large cutoff, we find $B_{3}=0.057 \mathrm{~K}$, but in the best case scenario corrections from higher orders should be very important.

Because of the similarity of the integral equations, our arguments should apply to systems of three fermions with internal quantum numbers as well. Preliminary results for the threenucleon system in the $J=1 / 2$ channel confirm this expectation and produce good $N d$ scattering and triton results [125]. The results of this section hold in the EFT where the pion has been integrated out, and can be straightforwardly generalized to the EFT with perturbative pions. Work in the latter is now in progress for the $J=3 / 2$ channel [129]. We also plan to extend these calculations to the four- and more-nucleon systems [125]. We have seen in this section how we need to introduce a three-body force in leading order to prevent the collapse of the three-body system that would result from purely attractive two-body contact interactions. Is this repulsion enough to allow a prediction of other few-body bound states?

\section{B. Moderate energies}

Increasing the range of the EFT, we affect the ultraviolet behavior of the leading-order two-body amplitude. As we hit $Q \sim M_{N N}$, contributions from pions become comparable to the leading contact interaction. The results of the previous subsection do not necessarily apply, as the two-body interaction is no longer purely attractive, but might display shortrange repulsion besides long-range attraction. Here I continue in the vein of Sect. $\mathbb{C} \mathrm{C}$, assuming that no enhancements appear in contact interactions. The power counting of that section can then be extended straightforwardly to an $A$-nucleon system.

Consider an arbitrary contribution to an irreducible graph involving $A$ nucleons. This being only part of the full amplitude, it will in general have $C$ separately connected pieces $(C=1$ for $A=0,1 ; C=1, \ldots, A-1$ for $A \geq 2$.) The straightforward generalization of Eq. (109) is 10,13]

$$
\nu=4-A+2(L-C)+\sum_{i} V_{i} \Delta_{i} .
$$

Again, because $C$ is bounded from above $\left(C \leq C_{\max }\right)$, the chiral symmetry constraint $\Delta_{i} \geq 0$ still implies a lower bound on the power of $Q, \nu \geq \nu_{\min }=4-A-2 C_{\max }$ for strong interactions, so that systematic calculations can be carried out.

With this power counting we can get some insight into few-body forces (those diagrams with $C=1$ ). The new forces that appear in systems with more than two nucleons have been considered in Refs. [12, 13, 15, 17]. The dominant potential, at $\nu=6-3 A=\nu_{\min }$, is simply the two-nucleon potential of lowest order that we have already encountered in Sect. IVQ. We can easily verify that a three-body potential will arise at $\nu=\nu_{\min }+2$, a four-body potential at $\nu=\nu_{\min }+4$, and so on. It is (approximate) chiral symmetry therefore that implies that $n$-nucleon forces $V_{n N}$ are expected to obey a hierarchy of the type 


$$
\left\langle V_{(n+1) N}\right\rangle /\left\langle V_{n N}\right\rangle \sim O\left(\left(Q / M_{Q C D}\right)^{2}\right),
$$

with $\left\langle V_{n N}\right\rangle$ denoting the contribution per $n$-plet. This is a non-trivial consequence of chiral symmetry, as there exist non-chiral models that produce large three-body forces. If $\left|\left\langle V_{2 N}\right\rangle\right| \sim$ $10 \mathrm{MeV}$ as in Sect. $\mathbb{V V}$, we can estimate $\left|\left\langle V_{3 N}\right\rangle\right| \sim 0.5 \mathrm{MeV},\left|\left\langle V_{4 N}\right\rangle\right| \sim 0.02 \mathrm{MeV}$, and so on. This is in accord with detailed few-nucleon calculations using phenomenological potentials.

It proves instructive to look at the form of the first few terms in the few-nucleon potential [12,15.17]. We do so with time-ordered perturbation theory, as before. At $\nu=\nu_{\min }+2$, in addition to corrections to the two-nucleon potential, one also finds diagrams involving either three nucleons or two pairs of nucleons connected via leading contact interactions and static pions. One finds [10] that the various orderings of Fig. 17(a) add to zero. Diagrams like those in Fig. 17(b,c) give non-vanishing contributions to three- and double-pair potentials. However, it is easy to prove [13,17] that these contributions cancel, to this order in the expansion, against contributions from reducible graphs like those in Fig. 177(d,e) involving the leading and the energy-dependent pieces of the two-nucleon potential. This happens because a small energy denominator combines with the small energy-dependent OPE to produce a result not only of the same order, but exactly opposite to the three- and doublepair potentials. This cancellation had been noted before in the case of the TPE $3 N$ force [130,99, but its model independence and generality are particularly clear in the EFT. Refs. 107.105 have emphasized that redefining the potential to eliminate energy dependence leads to no $3 N$ TPE forces of this type at all. Remaining, to this order, are only "true" three-body forces generated by the delta isobar, and at $\nu_{\min }+3$ further terms with similar structure arise [15.17] - see Fig. [18. Up to this order, there are no four-nucleon forces, and the three-nucleon potential has components with three different ranges: neglecting relativistic corrections,

$$
\begin{aligned}
V_{3}^{(3)}\left(\vec{q}_{i j}, \vec{q}_{j k}\right)= & e_{1} \boldsymbol{t}_{i} \cdot \boldsymbol{t}_{k}+e_{2} \vec{\sigma}_{i} \cdot \vec{\sigma}_{k} \boldsymbol{t}_{i} \cdot \boldsymbol{t}_{k}+e_{3} \vec{\sigma}_{j} \cdot\left(\vec{\sigma}_{i} \times \vec{\sigma}_{k}\right) \boldsymbol{t}_{j} \cdot\left(\boldsymbol{t}_{i} \times \boldsymbol{t}_{k}\right) \\
& -\frac{2 g_{A}}{F_{\pi}^{2}} \frac{1}{w_{j k}^{2}} \vec{\sigma}_{k} \cdot \vec{q}_{j k}\left[d_{1}\left(\boldsymbol{t}_{i} \cdot \boldsymbol{t}_{k} \vec{\sigma}_{i}+\boldsymbol{t}_{j} \cdot \boldsymbol{t}_{k} \vec{\sigma}_{j}\right)-2 d_{2} \boldsymbol{t}_{j} \cdot\left(\boldsymbol{t}_{i} \times \boldsymbol{t}_{k}\right) \vec{\sigma}_{i} \times \vec{\sigma}_{j}\right] \cdot \vec{q}_{j k} \\
+ & 8\left(\frac{2 g_{A}}{F_{\pi}^{2}}\right)^{2} \frac{1}{w_{i j}^{2} w_{j k}^{2}} \vec{\sigma}_{i} \cdot \vec{q}_{i j} \vec{\sigma}_{k} \cdot \vec{q}_{j k} \times \\
& \quad \times\left[\boldsymbol{t}_{i} \cdot \boldsymbol{t}_{k}\left(c_{3} \vec{q}_{i j} \cdot \vec{q}_{j k}+2 c_{1} m_{\pi}^{2}\right)+c_{4} \boldsymbol{t}_{j} \cdot\left(\boldsymbol{t}_{i} \times \boldsymbol{t}_{k}\right) \vec{\sigma}_{j} \cdot\left(\vec{q}_{i j} \times \vec{q}_{j k}\right)\right] \\
& + \text { two cyclic permutations of }(i j k),
\end{aligned}
$$

with $\vec{p}_{i}\left(\vec{p}_{i}^{\prime}\right)$ the initial (final) momentum of nucleon $i, \vec{q}_{i j} \equiv \vec{p}_{i}-\vec{p}_{i}^{\prime}=\vec{p}_{j}^{\prime}-\vec{p}_{j}$, and $w_{i j}^{2} \equiv$ $\vec{q}_{i j}^{2}+m_{\pi}^{2}$. The parameters $c_{i}$ and $d_{i}$ are given by Eqs. (54) and (102) in terms of the $B_{i}$ from Eq. (48), the $\pi N \Delta$ coupling $h_{A}$ from Eq. (47), the $D_{i}$ from Eq. (100), and the $N N N \Delta$ contact interaction $D_{T}$ from Eq. (99). Likewise, the $e_{i}$ are parameters that have delta and shorter-range components $E_{i}$,

$$
e_{1}=E_{1}, \quad e_{2}=E_{2}+\frac{D_{T}^{2}}{9 \delta m}, \quad e_{3}=E_{3}-\frac{D_{T}^{2}}{18 \delta m} .
$$

The TPE part of the potential (135) is determined in terms of $\pi N$ scattering observables. It is not identical to the Tucson-Melbourne potential [48]. The latter was built from 


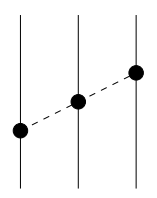

(a)

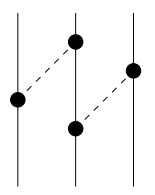

(b)

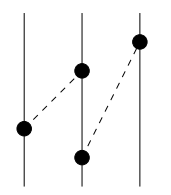

(c)

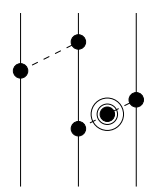

(d)

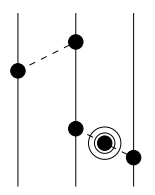

(e)

FIG. 17. Some time-ordered diagrams whose contributions add to nothing in the EFT: (a) TPE from the Weinberg-Tomozawa seagull, of which only one ordering is shown; (b,c) "uncorrelated" TPE three-nucleon potential and (d,e) iteration of energy-dependent OPE two-nucleon potential. Solid lines represent nucleons, dashed lines pions, a heavy dot an interaction in $\mathcal{L}^{(0)}$, and a dot within two circles an interaction in $\mathcal{L}^{(2)}$. The same cancellation (b-e) occurs in other sets of three-nucleon diagrams corresponding to other orderings. It also takes place in diagrams where short-range interactions are substituted for static OPE, and in sets of double-pair diagrams.

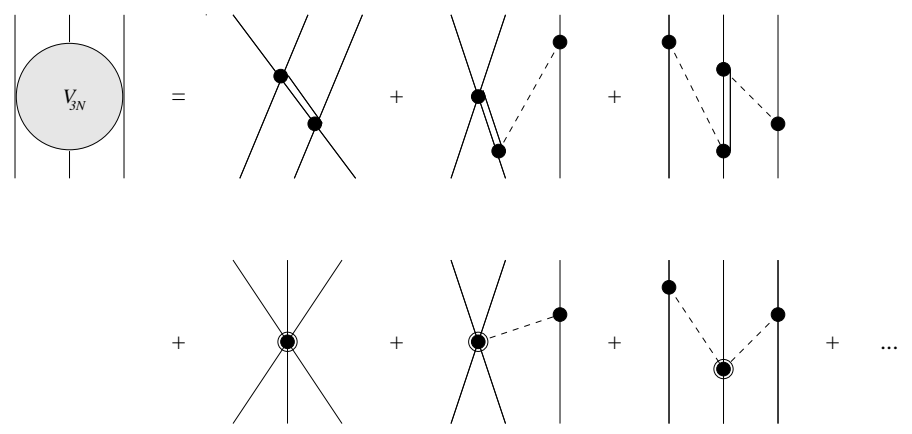

FIG. 18. Some time-ordered diagrams contributing to the three-nucleon potential $V_{3 N}$ in the EFT. (Double) solid lines represent nucleons (and/or deltas), dashed lines pions, a heavy dot an interaction in $\mathcal{L}^{(0)}$, and a dot within a circle an interaction in $\mathcal{L}^{(1)}$. First line corresponds to $\nu=\nu_{\min }+2$, second line to $\nu=\nu_{\min }+3$, and “..." denote $\nu \geq \nu_{\min }+4$. All nucleon permutations and orderings with at least one pion or delta in intermediate states are included.

$\pi N$ scattering through a particular choice of pion fields for which chiral symmetry is not respected term-by-term [131]. Although the corresponding on-shell $\pi N$ amplitude gives the same result as Eqs. (55) and (56), they differ off shell. The corresponding three-body potential involves other interactions to guarantee chiral symmetry and to enforce invariance under pion-field redefinitions, but unfortunately such terms have not been included in the original Tucson-Melbourne potential. After this is done, all commonly used TPE threenucleon potentials agree, except for the numerical values of parameters that depend on the way $\pi N$ data is fitted - see Table $\mathrm{V}$ [131]. The novel one-pion/short-range components of the potential (135) can be related to pion production in $N N$ collisions. They are starting to be explored in conjunction with more conventional treatments of few-nucleon systems [138,139. The purely short-range components can only be determined from few-nucleon systems, but to leading order depend only on one undetermined parameter, $D_{T}$. (Recall that $D_{T}$ could not be isolated in the $\nu=\nu_{\min }+3$ EFT fit of $N N$ phase shifts.) Relativistic corrections neglected above are discussed in Ref. [99]. 
TABLE V. Low-energy $\pi N$ scattering parameters (with Born terms removed) in a variety of existing TPE three-nucleon forces.

\begin{tabular}{c||cccc}
\hline \hline Three-Nucleon Force & $\left(a-2 m_{\pi}^{2} c\right) m_{\pi}$ & $b m_{\pi}^{3}$ & $c m_{\pi}^{3}$ & $d m_{\pi}^{3}$ \\
\hline Fujita-Miyazawa [132] & 0.0 & -1.15 & 0.0 & -0.29 \\
Tucson-Melbourne [18, 133] & -1.03 & -2.62 & 1.03 & -0.60 \\
Brazil [134,133] & -1.05 & -2.29 & 1.05 & -0.77 \\
Urbana-Argonne [135,136] & 0.0 & -1.20 & 0.0 & -0.30 \\
Texas [17,53] & -1.87 & -3.82 & 0.0 & -1.12 \\
Ruhr(Pot) [137] & -0.51 & -1.82 & 0.0 & -0.48 \\
\hline \hline
\end{tabular}

\section{PROBES OF FEW-NUCLEON SYSTEMS}

Further constraints on our understanding of few-nucleon systems come from the study of processes involving external probes, such as pions, photons, electrons and neutrinos. In some cases the calculation in the EFT to a certain order is completely determined by parameters already known from other processes; a prediction can be made, accompanied by an educated guess about the error resulting from neglecting higher orders. Other cases can be used to determine so-far unknown parameters; this is particularly useful in the case of neutron and neutral pion parameters that cannot be easily determined in processes with a single-nucleon target - see Sect. III for examples. But even in these cases, a prediction for energy dependence is usually possible.

\section{A. Very low and low energies}

In the EFT with virtual pions integrated out, scattering of pions on few-nucleon systems is in itself not very interesting, as pions behave just as heavy particles. More interesting are processes involving photons of momenta $Q \sim \aleph$, which we can use as efficient probes of momentum distributions. As we have seen, this EFT is equivalent in the two-nucleon sector to effective range theory. An extensive literature exists using this technique in the calculation of electroweak process involving the deuteron, starting with the pioneering work of Refs. [1.140]. These calculations can be recast in terms of the very-low-momentum EFT in a straightforward way. We briefly describe here some of the more modern calculations, carried out with explicit but perturbative pions, that is, in the low-momentum EFT. Calculations involving the two-nucleon system proceed by separating "irreducible" contributions, which are those that do not split in two when cut at any $C_{0}$ vertex. Always contributing is an irreducible two-nucleon function, intimately related to the two-nucleon amplitude. Other irreducible components include the external probes. Each irreducible part is calculated to the same order in the $Q / M_{N N}$ expansion, they are combined in the amplitude of the processes of interest, and only terms up to the desired power of $Q / M_{N N}$ are kept. These calculations typically contain the result of the lower-scale EFT; improvement due to the additional pion effects, if any, can be taken as evidence for $M_{N N}>m_{\pi}$.

- Deuteron form factors. A calculation of the deuteron form factors in subleading order was carried out in Ref. [141]. To this order the deuteron is pure $S$ wave, determined by 
TABLE VI. Results from EFT at leading (LO) and subleading (SLO) order and experimental values for deuteron rms charge radius $\left(\left\langle r^{2}\right\rangle_{c h}^{1 / 2}\right)$, magnetic dipole moment $\left(\mu_{d}\right)$, and electric quadrupole moment $\left(Q_{E}\right)$.

\begin{tabular}{c|ccc}
\hline \hline quantity & LO & NL+SLO & expt \\
\hline$\left\langle r^{2}\right\rangle_{c h}^{1 / 2}$ & 1.53 & 1.89 & $2.1303(66)$ \\
$\mu_{d}$ & 0.88 & $0.86(\mathrm{fit})$ & $0.857406(1)$ \\
$Q_{E}$ & 0 & 0.40 & $0.2859(3)$ \\
\hline \hline
\end{tabular}

the ${ }^{3} S_{1}$ parameters $C_{0}+m_{\pi}^{2} C_{2 q m}$ and $C_{2}$. Interactions with a photon field come, at this order, from known one-nucleon terms, from minimal substitution in the $C_{2}$ term, and from one a priori unknown two-body magnetic-interaction counterterm, which can only be fitted to data. With $C_{0}+m_{\pi}^{2} C_{2 q m}$ fitted to the deuteron binding and $C_{2}$ to the phase shifts, the results for static moments are in Table VI [141]. The momentum dependence of the charge and magnetic form factors can then be predicted. Good convergence and agreement with data are attained even at momenta as large as $\sim 400 \mathrm{MeV}$; only the quadrupole moment works poorly. Unfortunately no improvement over the effective range expansion is seen. Comparison with effective range theory for the radius and EFT with non-perturbative pions for the quadrupole moment suggest that the most important of the neglect terms are from iterated pion exchange [141]. Sub-subleading contributions to the quadrupole moment are discussed in Ref. 142]. The anapole moment of the deuteron was studied in Ref. [143.

- $n p \rightarrow \gamma d$ at threshold. To subleading order this reaction proceeds from the ${ }^{1} S_{0}$ state; the same set of ${ }^{1} S_{0}$ and ${ }^{3} S_{1}$ parameters $C_{0}+m_{\pi}^{2} C_{2 q m}$ and $C_{2}$ appear and are determined by phase shifts and deuteron binding. In leading order only one-body currents from magnetic coupling appear. At subleading order pion-exchange currents are calculated and found to be cutoff dependent; a four-nucleon/one-photon operator appears at the same order and absorbs this dependence leaving an undetermined finite part behind, which can then be fitted. Results [144 are presented in Table VII. The parity-violating asymmetry in the angular distribution of gamma rays from radiative capture of polarized cold neutrons was calculated in Ref. [145].

- $\gamma d \rightarrow \gamma d$. Amplitudes with two external photons give rise to interesting predictions. In leading non-vanishing order only one-nucleon operators from subleading interactions enter, including the Thomson seagull operator and interactions from minimal coupling. At subleading order pion exchange and $C_{2}$ appear as well. Scalar and tensor, electric and magnetic

TABLE VII. Values for the total cross-section $\sigma$ in mb for radiative neutron-proton capture: leading (LO) and subleading (SLO) order EFT and experiment (expt).

\begin{tabular}{llc}
\hline \hline $\mathrm{LO}$ & $\mathrm{NL}+\mathrm{SLO}$ & $\mathrm{expt}$ \\
\hline 297.2 & $334.2(\mathrm{fit})$ & $334.2 \pm 0.5$ \\
\hline \hline
\end{tabular}


TABLE VIII. Scalar and tensor electric $\left(\alpha_{0}\right.$ and $\left.\alpha_{2}\right)$ and magnetic $\left(\beta_{0}\right.$ and $\left.\beta_{2}\right)$ polarizabilities of the deuteron in $\mathrm{fm}^{3}$ : leading (LO) and subleading (SLO) order EFT

\begin{tabular}{ccc}
\hline \hline polarizability & LO & NL+SLO \\
\hline$\alpha_{0}\left(\mathrm{fm}^{3}\right)$ & 0.386 & 0.595 \\
$\alpha_{2}\left(\mathrm{fm}^{3}\right)$ & 0 & -0.062 \\
$\beta_{0}\left(\mathrm{fm}^{3}\right)$ & 0.067 & $?$ \\
$\beta_{2}\left(\mathrm{fm}^{3}\right)$ & 0.195 & $?$ \\
\hline \hline
\end{tabular}

polarizabilities have been computed in Ref. [146] and are shown in Table VIII. Convergence seems consistent with static electromagnetic moments, and results are comparable to the effective range expansion. Nucleon polarizability enters as a higher-order contribution, and therefore it is unlikely that it can be isolated from the deuteron polarizabilities. Differential cross-sections for Compton scattering at photon energies of 49 and $69 \mathrm{MeV}$ were calculated in Ref. [147]. The parameter-free results are in good agreement with existing data, in particular at the lower energy. Nucleon polarizabilities from pion loops enter to subleading order and are important for this agreement. Tensor polarized scattering was computed in Ref. 148. At $90^{\circ}$ the first non-vanishing contribution comes only from the pion exchange, and therefore might allow a sensitive test of the goodness of perturbative pions.

\section{B. Moderate energies}

The power counting arguments of Sect. $\mathrm{VB}$ can be easily generalized to the case where external particles with momenta $Q \sim M_{n u c}$ interact with few-nucleon systems. We define the kernel $K$ as the sum of irreducible diagrams to which the probes are attached. A generic diagram contributing to a full amplitude will consist of irreducible diagrams sewed together by states with small energy denominators. Interactions among nucleons occurring before or after scattering can be treated as before: iteration of the potential gives rise to the wave-function $|\psi\rangle\left(\left|\psi^{\prime}\right\rangle\right)$ of the initial (final) nuclear state. The full scattering amplitude is then

$$
T \sim\left\langle\psi^{\prime}|K| \psi\right\rangle
$$

The power counting (133) applies equally well to the kernel $K$. In practice, it is frequently desirable to minimize nuclear wave-function errors by using a high-precision phenomenological potential instead of the only currently available EFT potential [16]. That this is a good approximation is suggested by a comparison [88] between a simplified version of the EFT potential of Ref. [16] and the Argonne V18 potential [82, which show agreement in most of the wave-function features.

Before plunging into hard results, let me point out the generic results of this EFT. Because of the factor $-2 C$ in Eq. (133), we see immediately - in an effect similar to few-nucleon forces - that external probes will tend to interact predominantly with a single nucleon, simultaneous interactions with more than one nucleon being suppressed by powers 
of $\left(Q / M_{Q C D}\right)^{2}$. Again, this generic dominance of the impulse approximation is a well-known result that arises naturally here. This is of course what allows extraction, to a certain accuracy, of one-nucleon parameters from nuclear experiments. A valuable by-product of the EFT is to provide a consistent framework for one- and few-nucleon dynamics, where few-nucleon process can be used to infer one-nucleon properties. More interesting from the purely nuclear-dynamics perspective are, however, those processes where the leading singlenucleon contribution vanishes by a particular choice of experimental conditions, for example the threshold region. In this case, two-nucleon contributions, especially in the relatively large deuteron, can become important. Further examination of the structure of the chiral Lagrangian reveals that two-nucleon contributions tend to be dominated by pion exchange. Indeed, photons and pions couple to four-nucleon operators only at $O\left(Q / M_{Q C D}\right)$ relative to pion-exchange diagrams constructed out of the leading order Lagrangian. Thus power counting justifies the "chiral filter hypothesis" that has been put forward to summarize some empirical results on electroweak form factors [11]. This "pion dominance" ensures that two-body contributions from the EFT in lowest orders tend to be similar to those in phenomenological models that include pion-exchange currents.

- $\pi d \rightarrow \pi d$. This is perhaps the most direct way to check the consistency of $\chi \mathrm{PT}$ in one- and few-nucleon systems. For simplicity, consider the region near threshold. There the lowest-order, $\nu=\nu_{\min }=-2$ contributions to the kernel vanish because the pion is in an $S$ wave and the target is isoscalar. The $\nu=\nu_{\text {min }}+1$ term comes from the (small) isoscalar pion-nucleon seagull, related in lowest-order to the pion-nucleon isoscalar amplitude $b^{(0)}$. $\nu=\nu_{\min }+2, \nu_{\min }+3$ contributions come from corrections to $\pi N$ scattering and two-nucleon diagrams, which involve besides $b^{(0)}$ also the much larger isovector amplitude $b^{(1)}$. Weinberg 13 has estimated these various contributions to the $\pi d$ scattering length, finding agreement with previous, more phenomenological calculations, which have been used to extract $b^{(0)}$. The impact of the deuteron process on the determination of $b^{(0)}$ is further discussed in Ref. [149. The size of two-nucleon, one-loop diagrams that appear in next order is estimated to be small in the related, double-charge-exchange process [150]. Charge symmetry breaking effects are considered in Ref. [151].

- $n p \rightarrow \gamma d$. This offers a chance of a precise postdiction. Here it is the transverse nature of the real outgoing photon that is responsible for the vanishing of the lowest-order, $\nu=\nu_{\min }=-2$ contribution to the kernel. The single-nucleon magnetic contributions come at $\nu=\nu_{\text {min }}+1$ (tree level), $\nu=\nu_{\min }+2$ (one loop), etc. The first two-nucleon term is one-pion exchange at $\nu=\nu_{\min }+2$, long discovered to give a smaller but non-negligible contribution. There has been a longstanding discrepancy of a few percent between these contributions and experiment. At $\nu=\nu_{\min }+4$ there are further one-pion exchange, twopion exchange, and short-range terms. In Ref. 152 the two-pion exchange diagrams in the deltaless theory were calculated and resonance saturation used to estimate the other $\nu=\nu_{\min }+4$ terms. With wave-functions from the Argonne V18 potential [82] and a cut-off $\Lambda=1000 \mathrm{MeV}$, the excellent agreement with experiment shown in Table [X] was found [152]. The total cross-section changes by only $0.3 \%$ if the cut-off is decreased to $500 \mathrm{MeV}$.

- $\gamma d \rightarrow \gamma d$. External photons couple to the kernel only in subleading order, $\nu=\nu_{\min }+$ $1=-1$, via the one-body Thomson seagull. In next order, $\nu=\nu_{\min }+2$, more interesting effects are present, such as a pion loop which contributes to the nucleon polarizability. There are also two-nucleon contributions from pion exchange, which can and need to be computed, 
TABLE IX. Values for various contributions to the total cross-section $\sigma$ in mb for radiative neutron-proton capture: impulse approximation to $\nu=\nu_{\min }+4$ (imp), impulse plus two-nucleon diagrams at $\nu=\nu_{\min }+2(\mathrm{imp}+\mathrm{tn} 0)$, impulse plus two-nucleon diagrams up to $\nu=\nu_{\min }+4$ (imp+tn), and experiment (expt).

\begin{tabular}{lccc}
\hline \hline imp & imp + tn0 & imp + tn & expt \\
\hline 305.6 & 321.7 & 336.0 & $334 \pm 0.5$ \\
\hline \hline
\end{tabular}

if one seeks to extract information about the neutron polarizability. Work is in progress [153] to calculate the differential cross-section at 49, 69, and $95 \mathrm{MeV}$. Preliminary results are in good agreement with the existing Illinois data at the lowest two energies, and a prediction will be made for the highest energy, currently being measured and analyzed in Saskatoon.

- $\gamma d \rightarrow \pi^{0} d$. This reaction offers the possibility to test a prediction arising from a combination of two-nucleon contributions and the neutral-pion single-neutron amplitude. The differential cross-section for a photon of momentum $k$ to produce a pion of momentum $q$ is, at threshold, $[(k / q)(d \sigma / d \Omega)]_{q=0}=8\left|E_{d}\right|^{2} / 3$. $E_{d}$ has been studied up to $\nu=\nu_{\min }+3$ with the delta integrated out in Ref. [154]. There are two classes of contributions, according to whether the external light particles interact with one or with both nucleons. The onenucleon part of the kernel is given by the same $\nu=\nu_{\min }, \nu_{\min }+1, \ldots$ mechanisms described in Sect. III, with due account of $P$ waves and Fermi motion inside the deuteron. The neutrality of the outgoing $S$-wave pion ensures that the leading $\nu=-2=\nu_{\min }$ terms vanish. The first two-nucleon part of the kernel appears at $\nu=\nu_{\text {min }}+2$; it comes from a virtual charged pion photoproduced on one nucleon which rescatters on the other nucleon with charge exchange. These contributions are actually numerically larger than indicated by power counting due to relatively large deuteron size. Smaller two-nucleon terms appear at $\nu=\nu_{\text {min }}+3$ from corrections in either nucleon. Results for $E_{d}$ up to $\nu=\nu_{\min }+3$ [154 are shown in Table Х. They correspond to the Argonne V18 potential [82] and a cutoff $\Lambda=1000 \mathrm{MeV}$. Other realistic potentials and cutoffs from 650 to $1500 \mathrm{MeV}$ give the same result within $5 \%$. The chiral potential of Sect. IVC is more cumbersome to use, but it has been verified that it gives $\nu=\nu_{\min }+2$ results that are similar to other realistic potentials. We see that two-nucleon contributions seem to be converging, although more convincing evidence would come from next order, where loops appear. A model-dependent estimate 155 of some $\nu=\nu_{\min }+4$ terms suggests a $10 \%$ or larger error from neglected higher orders in the kernel itself. The single-scattering amplitude depends on amplitude for $\gamma n \rightarrow \pi^{0} n, E_{0+}\left(\pi^{0} n\right)$, in such a way that $E_{d} \sim-1.79-0.38\left(2.13-E_{0+}\left(\pi^{0} n\right)\right)$ in units of $10^{-3} / m_{\pi^{+}}$. Thus, sensitivity to $E_{0+}\left(\pi^{0} n\right)$ survives the large two-nucleon contribution at $\nu=\nu_{\min }+2$.

We see that working within the effective theory yields a testable prediction, $E_{d}=$ $-(1.8 \pm 0.2) \cdot 10^{-3} / m_{\pi^{+}}$. It is remarkable that for this process $\chi \mathrm{PT}$ gives results that are somewhat different from tree-level models of the type traditionally used in nuclear physics. For example, the models in Ref. [156] predict the threshold cross-section about twice as large as $\chi$ PT. Most of the difference comes from one-nucleon loop diagrams: tree-level models tend to differ from $\chi \mathrm{PT}$ mostly by having a smaller $E_{0+}\left(\pi^{0} n\right)$, which increases $\left|E_{d}\right|$. A test of this prediction is thus an important check of our understanding of the role of QCD at low 


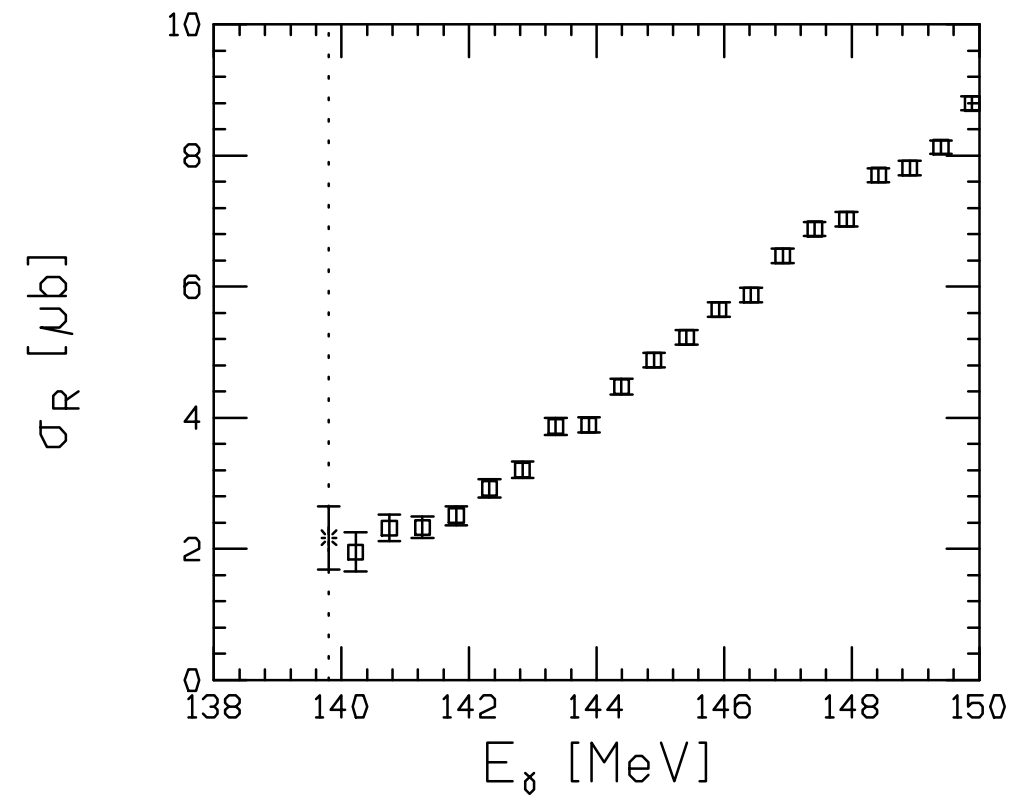

FIG. 19. Reduced cross-section $\sigma_{R}=(k / q) \sigma$ in $\mu \mathrm{b}$ for neutral pion photoproduction as function of the photon energy in $\mathrm{MeV}$. Threshold is marked by a dotted line. Squares are data points from Ref. 157] and the star at threshold is the $\chi \mathrm{PT}$ prediction of Ref. [154]. Figure courtesy of U.-G. Meißner.

energies. Such a test was recently carried out at Saskatoon [157]. The experimental results for the pion photoproduction cross-section near threshold are shown in Fig. 19, together with our $\chi \mathrm{PT}$ prediction at threshold. Inelastic contributions have been estimated in Ref. 157 and are smaller than $10 \%$ throughout the range of energies shown. At threshold, Ref. 157 finds $E_{d}=-(1.45 \pm 0.09) \cdot 10^{-3} / m_{\pi^{+}}$. While agreement with $\chi \mathrm{PT}$ to order $\nu=\nu_{\min }+3$ is not better than a reasonable estimate of higher-order terms, it is clearly superior to treelevel models. This is compelling evidence of chiral loops. Further test will come from an electroproduction experiment currently under analysis in Mainz [158].

- Axial currents. The long-range two-nucleon contributions to the axial current come from one-pion exchange in first non-vanishing order and from two-pion exchange as a first correction. They have been evaluated in Ref. [14]. The related proton-burning process $p p \rightarrow d e^{+} \nu_{e}$ is found [87] in agreement with previous calculations.

- $p p \rightarrow p p \pi^{0}$ close to threshold. This reaction has attracted a lot of interest because

TABLE X. Values for $E_{d}$ in units of $10^{-3} / m_{\pi^{+}}$from one-nucleon contributions $(1 N)$ up to $\nu=\nu_{\min }+3$, two-nucleon kernel $(2 N)$ at $\nu=\nu_{\min }+2$ and at $\nu=\nu_{\min }+3$, and their sum $(1 N+2 N)$.

\begin{tabular}{cccc}
\hline \hline \multirow{2}{*}{$1 N$} & \multicolumn{2}{c}{$2 N$} & $1 N+2 N$ \\
$\nu \leq \nu_{\min }+3$ & $\nu=\nu_{\min }+2$ & $\nu=\nu_{\min }+3$ & $\nu \leq \nu_{\min }+3$ \\
\hline 0.36 & -1.90 & -0.25 & -1.79 \\
\hline \hline
\end{tabular}




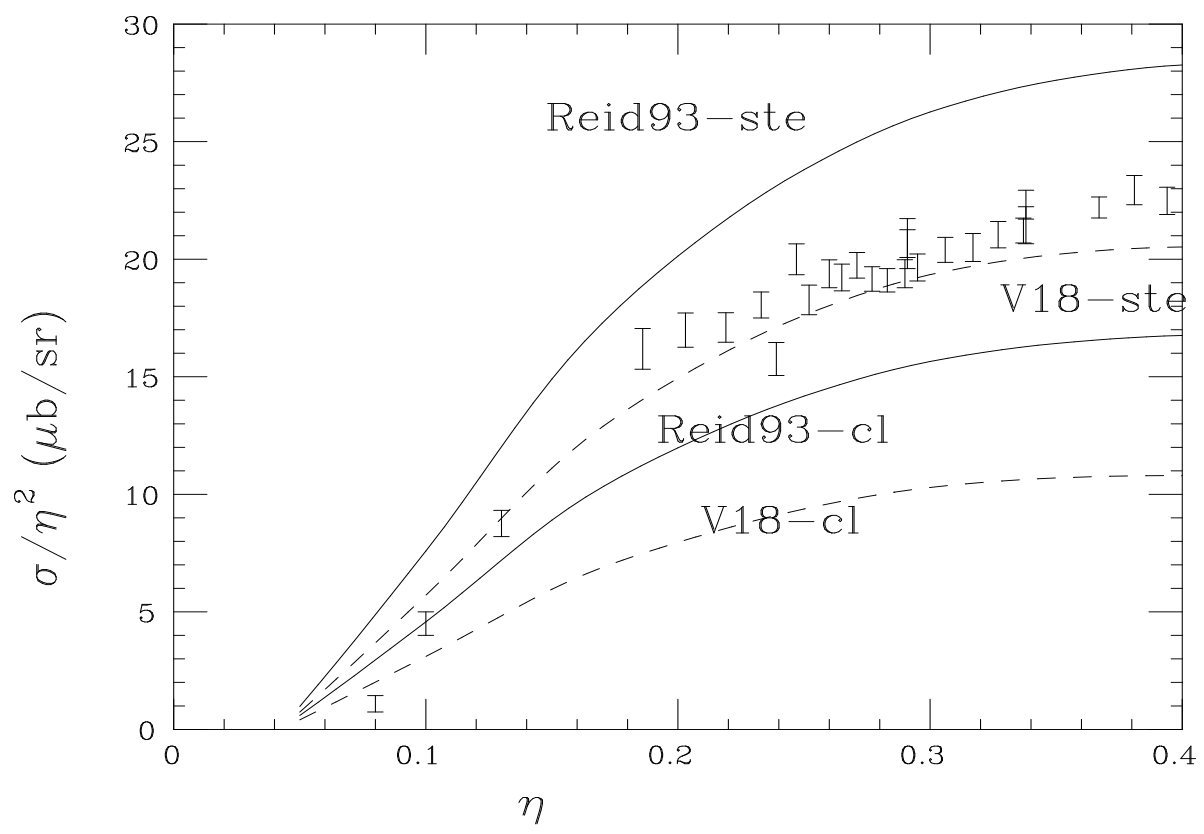

FIG. 20. Cross-section for $p p \rightarrow p p \pi^{0}$ in $\mu \mathrm{b} / \mathrm{sr}$ as function of the pion momentum $\eta$ in units of $m_{\pi}$ for two $N N$ potentials (Argonne V18 and Reid93) and two parametrizations of the isoscalar pion-nucleon amplitude (ste and $\mathrm{cl}$ ).

of the failure of standard phenomenological mechanisms in reproducing the small crosssection observed near threshold. It involves larger momenta of $O\left(\sqrt{m_{\pi} m_{N}}\right)$, so the relevant expansion parameter here is the not so small $\left(m_{\pi} / m_{N}\right)^{\frac{1}{2}}$. This process is therefore not a good testing ground for the above ideas. But $\left(m_{\pi} / m_{N}\right)^{\frac{1}{2}}$ is still $<1$, so at least in some formal sense we can perform a low-energy expansion. In Ref. 43] the chiral expansion was adapted to this reaction and the first few contributions estimated. (Note that - contrary to what is stated in Ref. [159]- momenta $\sim \sqrt{m_{\pi} m_{N}}$ do not necessarily imply a breakdown of the non-relativistic expansion, as $p^{4} / m_{N}^{3} \sim\left(m_{\pi} / m_{N}\right)\left(p^{2} / m_{N}\right)$.) Again, the lowest order terms all vanish. The formally leading non-vanishing terms - an impulse term and a similar diagram from the delta isobar - are anomalously small and partly cancel. The bulk of the cross-section must then arise from contributions that are relatively unimportant in other processes. One is isoscalar pion rescattering for which two sets of $\chi \mathrm{PT}$ parameters were used: "ste" from a sub-threshold expansion of the $\pi N$ amplitude [50] and "cl" from an oneloop analysis of threshold parameters 44]. Others are two-pion exchange and short-range $\pi N N N N$ terms, which were modeled by heavier-meson exchange: pair diagrams with $\sigma$ and $\omega$ exchange, and a $\pi \rho \omega$ coupling, among other, smaller terms 160. Two potentials Argonne V18 [82] and Reid93 [161] - were used. Results [160] are shown in Fig. 20 together with IUCF [162 and Uppsala [163] data. Other $\chi \mathrm{PT}$ studies of this reaction can be found in Ref. [164]. The situation here is clearly unsatisfactory, and presents therefore a unique window into the nuclear dynamics. Work is in progress, for example, on a similar analysis for the other, not so suppressed channels $\rightarrow d \pi^{+}, \rightarrow$ pn $\pi^{+}$165. 


\section{MANY-NUCLEON SYSTEMS}

The density of a many-body system sets the scale of typical particle momenta $Q$. At sufficiently low density such that $Q<\aleph$, we have seen that the EFT can be formulated in terms of contact interactions only, and should be perturbative. There is a vast literature on dilute systems (see, e.g. Ref. [23]). Most of its results can be rederived in an EFT language. For example, Ref. [166] has reexamined some of the higher corrections to the energy density of a dilute boson gas; and Ref. [167] has studied pairing in a dilute Fermi system, producing analytical expressions relating the pairing gap, the density, and the energy density to the scattering length. Only exploratory work has so far been carried out regarding the more challenging, higher densities where $Q / \aleph \gtrsim 1$. This should not be surprising in view of the non-trivial aspects that even the three-body system bears, as discussed in Sect. VA. Attempts to look at nuclear matter at equilibrium density and at finite nuclei have been phenomenological; within its limited scope, this pioneering work has nevertheless uncovered remarkable systematics.

It was very quickly realized that chiral Lagrangians could be related to Walecka-type models. Under the assumptions that pion and spin effects average to very little in heavy nuclei, the main interactions should be given by contact interactions. Under the further approximation of mean field, two four-nucleon contact interactions can indeed be related to the original Walecka model, with parameters of the correct order of magnitude [168]. More elaborate versions of the Walecka model can be shown to require further contact interactions. The more extensive analysis of Ref. [169] has shown a remarkable regularity in the size of parameters in a relativistic point-coupling model that includes several four-, six-, and eight-nucleon interactions, is treated in Dirac-Hartree mean-field, and is fitted to a large number of nuclear data. This regularity was confirmed and extended to more complete versions of the same model [170], and was shown to also hold in non-relativistic, Skyrme-force models [171]. It was found that a $2 n$-nucleon field operator without derivatives contributes $\sim\left(4 \rho_{0} / F_{\pi}^{2} M_{Q C D}\right)^{n-1} M_{Q C D}$ to the energy per particle at equilibrium density $\rho_{0}$. This is tantalizingly reminiscent of our previous naturalness assumptions. It has also been noted 171 that the cancellation between large scalar and vector densities that is characteristic of relativistic point-coupling models is incorporated automatically in a smaller contribution in the non-relativistic models. Although these models do not include all operators consistent with symmetries to a certain order in a definite power counting, additions of new interactions with natural parameters could change the precise values of all existing parameters but not their orders of magnitude. This regularity is a nontrivial result because at mean-field level effective interactions absorb long distance many-body effects from ladder and ring diagrams [172. It implies a convergent density expansion for mean-field contributions to nuclear matter, as the expansion parameter is $4 \rho_{0} / F_{\pi}^{2} M_{Q C D} \sim 0.2$ [19]. For an attempt at an EFT expansion around $\rho_{0}$, see Ref. [173]. This all hints that a controlled EFT expansion for finite density nuclear systems is possible. 


\section{OUTLOOK}

We have gone a long way in identifying the essential ingredients of the EFT appropriate for nuclear physics.

- In the two-nucleon system, the way fine-tuning surfaces in contact interactions, the role of a potential and of a dimeron field, and the virtues and limitations of various regulators have been understood. As a consequence, systematic calculations can and have been performed, in some cases producing simple analytical results. Two-nucleon observables work well at low energies, but not yet at the level of phenomenological potential models.

- In the three-nucleon system, we have learned about the subtlety of renormalization in a non-perturbative context and started exploring the rich features of phenomenology and three-body forces. Universal features — such as the Phillips line - arise as consequence of the renormalization group.

- Nuclear processes involving external particles have been treated consistently with onenucleon $\chi \mathrm{PT}$, providing a way to access neutron properties. In most cases results consistent with conventional models have been obtained, but in one case there was a prediction in disagreement with these models. This has stimulated experimental efforts which unequivocally favor the EFT.

- Evidence for naturalness has been unearthed in many-nucleon systems.

When these developments are taken together, a simple physics picture of nuclei emerges from QCD. Low-momentum nucleons can be thought of as point particles surrounded by $(i)$ an inner cloud which is dense but of short range $O\left(1 / M_{Q C D}\right)$, and (ii) an outer cloud made out of pions which has long range $O\left(1 / M_{n u c}\right)$ but is sparse. If a few non-relativistic nucleons are put together, each nucleon is unable to distinguish details of the others' inner clouds. Interactions in the inner region can be expanded in delta functions and their derivatives, of progressively smaller importance. The outer cloud yields interactions which are nonanalytic, but chiral symmetry guarantees that the chance of finding $n$ pions in the air at the same time decreases rapidly with $n$. The systematic theory built on this simple picture has a finite number of interactions at any desired accuracy. It in fact explains many regularities that have been discovered in previous phenomenological treatments but whose origins are otherwise mysterious. These include the decreasing importance of many-pion exchanges, many-nucleon forces, and higher isospin classes.

This picture is not in contradiction with potential-model experience. In fact, the method of EFT has already provided quantitative input for more standard analyses that could not have been obtained before by other means. The chiral TPE two-nucleon force and simultaneous pion-gamma exchange have been successfully incorporated in the Nijmegen phase shift analysis; chiral TPE three-nucleon forces have been used to correct existing models; and, at the same time, the effects of the shorter-range three-nucleon forces on discrepancies remaining in conventional approaches are starting to be explored. From a conservative point-of-view, the EFT method can be considered a refinement of other hadronic 
approaches in that it brings to an otherwise free-fantasy context the constraints of QCD symmetries.

There is no denying that much is still to be done in raising this pre-teen EFT formulation of nuclear physics into a fully mature subject. One major source of malnourishment has been the so-far elusive determination of the scale where pion effects cease to be perturbative, and the scope of their resummations. Yet, this is a problem that can and will be resolved. A coherent description of few- and many-nucleon systems is still green but we can already glimpse the shape it is going to take.

\section{Acknowledgments}

The ideas reviewed here evolved from conversations with a large number of friends, collaborators, and colleagues. I thank them all, but particularly Silas Beane, Paulo Bedaque, and Jim Friar for their continuous input along the years. Helpful discussions on specific issues reported here are also acknowledged: Paulo Bedaque on $\aleph$ counting and the dimeron, Paulo Bedaque and Rob Timmermans on electromagnetic interactions, and Tom Cohen on perturbative pions. I am grateful to Silas Beane for helpful comments on the manuscript, to Ulf Meißner, Dan Phillips, and Martin Savage for allowing me to use their figures, and to Jim Friar, Barry Holstein, Tom Mehen, Gautam Rupak, Iain Stewart, and Rob Timmermans for making their unpublished papers available to me. This research was supported in part by the US National Science Foundation, grant PHY 94-20470. 


\section{REFERENCES}

[1] H. Bethe and R. Peierls, Proc. Roy. Soc. A148 (1935) 146; A149 (1935) 176.

[2] W. Heisenberg and H. Euler, Z. Phys. 98 (1936) 714.

[3] S. Weinberg, Phys. Rev. Lett. 18 (1967) 188.

[4] H. Georgi and S.L. Glashow, Phys. Rev. Lett. 32 (1974) 438; J. Pati and A. Salam, Phys. Rev. D10 (1974) 275.

[5] K.G. Wilson and J.G. Kogut, Phys. Rep. 12 (1974) 75.

[6] S. Weinberg, Physica 96A (1979) 327.

[7] S. Weinberg, Rev. Mod. Phys. 52 (1980) 515.

[8] J. Gasser and H. Leutwyler, Ann. Phys. 158 (1984) 142; Nucl. Phys. B250 (1985) 465.

[9] J. Gasser, M.E. Sainio, and A. Švarc, Nucl. Phys. B307 (1988) 779.

[10] S. Weinberg, Phys. Lett. B251 (1990) 288; Nucl. Phys. B363 (1991) 3.

[11] M. Rho, Phys. Rev. Lett. 66 (1991) 1275.

[12] C. Ordóñez and U. van Kolck, Phys. Lett. B291 (1992) 459.

[13] S. Weinberg, Phys. Lett. B295 (1992) 114.

[14] T.-S. Park, D.-P. Min, and M. Rho, Phys. Rep. 233 (1993) 341.

[15] U. van Kolck, Texas Ph.D. Dissertation (1993).

[16] C. Ordóñez, L. Ray and U. van Kolck, Phys. Rev. Lett. 72 (1994) 1982; Phys. Rev. C53 (1996) 2086.

[17] U. van Kolck, Phys. Rev. C49 (1994) 2932.

[18] U. van Kolck, Few-Body Syst. Suppl. 9 (1995) 444; Washington preprint DOE/ER/40427-13-N94 in preparation.

[19] J.L. Friar, Few-Body Syst. 22 (1997) 161.

[20] Nuclear Physics with Effective Field Theory, ed. R. Seki, U. van Kolck, and M.J. Savage (World Scientific, 1998).

[21] D.B. Kaplan, nucl-th/9901003; M. Rho, nucl-th/9812012 v2; M.J. Savage, nucl-th/9807023; S.R. Beane, Acta Phys. Polon. B29 (1998) 3161; U. van Kolck, Nucl. Phys. A631 (1998) 56c.

[22] D.B. Kaplan, nucl-th/9506035.

[23] Functional Integrals and Collective Excitations, V. N. Popov (Cambridge, 1987).

[24] J. Polchinski, in Recent Directions in Particle Theory: from Superstrings and Black Holes to the Standard Model, TASI'92, ed. J. Harvey and J. Polchinski (World Scientific, 1993).

[25] G.P. Lepage, in From Actions to Answers, TASI'89, ed. T. DeGrand and D. Toussaint (World Scientific, 1990).

[26] H. Georgi, Ann. Rev. Part. Sci. 43 (1994) 209.

[27] T.D. Lee and C.N. Yang, Phys. Rev. 128 (1962) 885.

[28] A. Salam and J. Strathdee, Phys. Rev. D2 (1970) 2869.

[29] J.M. Charap, Phys. Rev. D2 (1970) 1554; D3 (1971) 1998; J. Honerkamp and K. Meetz, Phys. Rev. D3 (1971) 1996; I. Gerstein, R. Jackiw, B.W. Lee, and S. Weinberg, Phys. Rev. D3 (1971) 2486.

[30] T. Appelquist and J. Carazzone, Phys. Rev. D11 (1975) 2856.

[31] J. Goldstone, A. Salam, and S. Weinberg, Phys. Rev. 127 (1962) 965.

[32] P. Higgs, Phys. Rev. Lett. 12 (1964) 132; F. Englert and R. Brout, Phys. Rev. Lett. 
13 (1964) 321; G.S. Guralnik, C.R. Hagen, and T.W.B. Kibble, Phys. Rev. Lett. 13 (1964) 585.

[33] R.D. Ball and R.S. Thorne, Ann. Phys. 236 (1994) 117.

[34] H. Georgi, Phys. Lett. B240 (1990) 447; E. Jenkins and A.V. Manohar, Phys. Lett. B255 (1991) 558.

[35] M. Luke and A.V. Manohar, Phys. Lett. B286 (1992) 348.

[36] S. Coleman, J. Wess, and B. Zumino, Phys. Rev. 177 (1969) 2239; C.G. Callan, S. Coleman, J. Wess, and B. Zumino, Phys. Rev. 177 (1969) 2247; S. Weinberg, in Lectures on Elementary Particles and Quantum Field Theory, ed. S. Deser et al. (MIT, 1970).

[37] J. Bijnens, Int. J. Mod. Phys. A8 (1993) 3045.

[38] A. Pich and E. de Rafael, Nucl. Phys. B367 (1991) 313.

[39] Dynamics of the Standard Model, J.F. Donoghue, E. Golowich, and B.R. Holstein (Cambridge, 1994).

[40] Quantum Field Theory, C. Itzykson and J.-B. Zuber (McGraw-Hill, 1985).

[41] G.'t Hooft, in Recent Developments in Gauge Theories, Cargèse 1979, ed. G.'t Hooft et al. (Plenum, 1980).

[42] G. Ecker, J. Gasser, A. Pich, and E. de Rafael, Nucl. Phys. B321 (1989) 311; J.F. Donoghue, C. Ramirez, and G. Valencia, Phys. Rev. D39 (1989) 1947.

[43] T.D. Cohen, J.L. Friar, G.A. Miller, and U. van Kolck Phys. Rev. C53 (1996) 2661.

[44] V. Bernard, N. Kaiser, and U.-G. Meißner, Int. J. Mod. Phys. E4 (1995) 193.

[45] S. Weinberg, Trans. N.Y. Acad. Sci. 38 (1977) 185.

[46] G. Ecker, Prog. Part. Nucl. Phys. 36 (1996) 71.

[47] E. Jenkins and A. Manohar, Phys. Lett. B259 (1991) 353; T.R. Hemmert, B.R. Holstein, and J. Kambor, Phys. Lett. B395 (1997) 89; J. Phys. G24 (1998) 1831.

[48] S.A. Coon, M.D. Scadron, P.C. McNamee, B.R. Barret, D.W.E. Blatt, and B.H.J. McKellar, Nucl. Phys. A317 (1979) 242.

[49] S. Weinberg, Phys. Rev. Lett. 17 (1966) 616; Y. Tomozawa, Nuovo Cim. 46A (1966) 707.

[50] V. Bernard, N. Kaiser, and U.-G. Meißner, Nucl. Phys. B457 (1995) 147.

[51] V. Bernard, N. Kaiser, and U.-G. Meißner, Nucl. Phys. A615 (1997) 483.

[52] M. Mojžiš, Eur. Phys. J. C2 (1998) 181.

[53] N. Fettes, U.-G. Meißner, and S. Steininger, Nucl. Phys. A640 (1998) 199.

[54] N. Fettes, U.-G. Meißner, and S. Steininger, hep-ph/9811366.

[55] V. Bernard, N. Kaiser, and U.-G. Meißner, Phys. Lett. B378 (1996) 337; Phys. Lett. B383 (1996) 116.

[56] U. van Kolck, in Proceedings of the Workshop on Chiral Dynamics 1997, Theory and Experiment, ed. A. Bernstein, D. Drechsel, and T. Walcher (Springer-Verlag, 1998), hep-ph/9711222; in Ref. [2]; Nucl. Phys. A645 (1999) 273.

[57] H.A. Bethe, Phys. Rev. 76 (1949) 38.

[58] See, e.g., Collision Theory, M.L. Goldberger and K.M. Watson (Krieger, 1975).

[59] J. Lekner, Mol. Phys. 23 (1972) 619.

[60] D.B. Kaplan, M.J. Savage, and M.B. Wise, Phys. Lett. B424 (1998) 390; Nucl. Phys. B534 (1998) 329.

[61] J. Gegelia, nucl-th/9802038; nucl-th/9805008 v2. 
[62] D.B. Kaplan, Nucl. Phys. B494 (1997) 471.

[63] E. Fermi, Ric. Scientifica 7 (1936) 13.

[64] G. Breit, Phys. Rev. 71 (1947) 215.

[65] K. Huang and C.N. Yang, Phys. Rev. 105 (1957) 767.

[66] V. Bargmann, Rev. Mod. Phys. 21 (1949) 488.

[67] J.V. Steele and R.J. Furnstahl, Nucl. Phys. A637 (1998) 46.

[68] M. Luke and A. Manohar, Phys. Rev. D55 (1997) 4129.

[69] M.C. Birse, J.A. McGovern, and K.G. Richardson, hep-ph/9807302; hep-ph/9808398.

[70] G.P. Lepage, nucl-th/9706029.

[71] D.R. Phillips, S.R. Beane, and T.D. Cohen, Ann. Phys. 263 (1998) 255.

[72] T.D. Cohen, Phys. Rev. C55 (1997) 67; D.R. Phillips and T.D. Cohen, Phys. Lett. B390 (1997) 7; K.A. Scaldeferri, D.R. Phillips, C.-W. Kao, and T.D. Cohen, Phys. Rev. C56 (1997) 679; S.R. Beane, T.D. Cohen, and D.R. Phillips, Nucl. Phys. A632 (1998) 445; K.G. Richardson, M.C. Birse, and J.A. McGovern, hep-ph/9708435.

[73] E.P. Wigner, Phys. Rev. 98 (1955) 145.

[74] D.B. Kaplan, private communication (1997).

[75] D.R. Phillips, S.R. Beane, and M.C. Birse, hep-th/9810049.

[76] D.B. Kaplan, M.J. Savage, and M.B. Wise, Nucl. Phys. B478 (1996) 629.

[77] M. Lutz, talk at the Workshop on the Standard Model at Low Energies, ECT*, Trento, May 1996, in hep-ph/9606301; private communications $(1996,1997)$.

[78] V.G.J. Stoks, R.A.M. Klomp, M.C.M. Rentmeester, and J.J. de Swart, Phys. Rev. C48 (1993) 792.

[79] G.A. Miller, B.M.K. Nefkens, and I. Šlaus, Phys. Rep. 194 (1990) 1.

[80] J.J. de Swart, C.P.F. Terheggen, and V.G.J. Stoks, nucl-th/9509032.

[81] D.R. Phillips, in Ref. [20].

[82] R.B. Wiringa, V.G.J. Stoks, and R. Schiavilla, Phys. Rev. C51 (1995) 38.

[83] T.-S. Park, hep-ph/9803417; T.-S. Park, K. Kubodera, D.-P. Min, and M. Rho, Phys. Rev. C58 (1998) 637.

[84] P.F. Bedaque and U. van Kolck, unpublished (1998).

[85] R. Timmermans, private communication (1998).

[86] X. Kong and F. Ravndal, nucl-th/9811076; B.R. Holstein, nucl-th/9901041.

[87] T.-S. Park, K. Kubodera, D.-P. Min, and M. Rho, astro-ph/9804144 v3.

[88] T.-S. Park, K. Kubodera, D.-P. Min, and M. Rho, nucl-th/9807054 v2.

[89] T.D. Cohen and J.M. Hansen, Phys. Lett. B440 (1998) 233.

[90] T.D. Cohen and J.M. Hansen, Phys. Rev. C59 (1999) 13.

[91] T.D. Cohen and J.M. Hansen, nucl-th/9901065.

[92] Pions and Nuclei, T. Ericson and W. Weise (Oxford, 1988).

[93] N. Kaiser, R. Brockmann, and W. Weise, Nucl. Phys. A625 (1997) 758; N. Kaiser, S. Gerstendörfer, and W. Weise, Nucl. Phys. A637 (1998) 395.

[94] J. Gegelia, nucl-th/9806028 v2.

[95] J.V. Steele and R.J. Furnstahl, Nucl. Phys. A645 (1999) 439.

[96] T. Mehen and I.W. Stewart, nucl-th/9809071; nucl-th/9809095.

[97] T. Mehen and I.W. Stewart, nucl-th/9901064.

[98] G. Rupak and N. Shoresh, in progress; S. Fleming, T. Mehen, and I.W. Stewart, in progress. 
[99] S.A. Coon and J.L. Friar, Phys. Rev. C34 (1986) 1060.

[100] J.L. Friar, Czech. J. Phys. 43 (1993) 259.

[101] E. Epelbaoum, W. Glöckle, and U.-G. Meißner, Phys. Lett. B439 (1998) 1; E. Epelbaoum, W. Glöckle, A. Krüger, and U.-G. Meißner, Nucl. Phys. A645 (1999) 413.

[102] K.A. Brueckner and K.M. Watson, Phys. Rev. 92 (1953) 1023.

[103] H. Sugawara and F. von Hippel, Phys. Rev. 172 (1968) 1764.

[104] M. Sugawara and S. Okubo, Phys. Rev. 117 (1960) 605, 611.

[105] E. Epelbaoum, W. Glöckle, and U.-G. Meißner, Nucl. Phys. A637 (1998) 107.

[106] J.L. Friar, nucl-th/9901082.

[107] J.L. Friar and S.A. Coon, Phys. Rev. C49 (1994) 1272.

[108] M. Taketani, S. Machida, and S. Ohnuma, Prog. Theor. Phys. 7 (1952) 45; S. Machida, Prog. Theor. Phys. Suppl. 39 (1967) 91.

[109] L.S. Celenza, A. Pantziris, and C.M. Shakin, Phys. Rev. C46 (1992) 2213; C.A. da Rocha and M.R. Robilotta, Phys. Rev. C49 (1994) 1818; Phys. Rev. C52 (1995) 531; Nucl. Phys. A615 (1997) 391; J.-L. Ballot, M.R. Robilotta, and C.A. da Rocha, Int. J. Mod. Phys. E6 (1997) 83; Phys. Rev. C57 (1998) 1574.

[110] M.C.M. Rentmeester, R.G.E. Timmermans, J.L. Friar, and J.J. de Swart. nucl-th/9901054.

[111] R.G.E. Timmermans, $\pi N$ Newsletter 13 (1997) 80.

[112] U. van Kolck, M.C.M. Rentmeester, J.L. Friar, T. Goldman, and J.J. de Swart, Phys. Rev. Lett. 80 (1998) 4386.

[113] J.L. Friar, T. Goldman, and U. van Kolck, in preparation

[114] U. van Kolck, J.L. Friar, and T. Goldman, Phys. Lett. B371 (1996) 169.

[115] S.A. Coon, B.H.J. McKellar, and V.G.J. Stoks, Phys. Lett. B385 (1996) 25.

[116] P.F. Bedaque and U. van Kolck, Phys. Lett. B428 (1998) 221.

[117] P.F. Bedaque, H.-W. Hammer, and U. van Kolck, Phys. Rev. C58 (1998) R641.

[118] P.F. Bedaque, H.-W. Hammer, and U. van Kolck, Phys. Rev. Lett. 82 (1999) 463;

Nucl. Phys. A to appear, nucl-th/9811046.

[119] G.V. Skorniakov and K.A. Ter-Martirosian, Sov. Phys. JETP 4 (1957) 648.

[120] W.T.H. van Oers and J.D. Seagrave, Phys. Lett. B24 (1967) 562.

[121] W. Dilg, L. Koester, and W. Nistler, Phys. Lett. B36 (1971) 208.

[122] G.S. Danilov and V.I. Lebedev, Sov. Phys. JETP 17 (1963) 1015.

[123] V.F. Kharchenko, Sov. J. Nucl. Phys. 16 (1973) 173.

[124] A.C. Phillips, Nucl. Phys. A107 (1968) 209.

[125] P.F. Bedaque, H.-W. Hammer, and U. van Kolck, in progress.

[126] F. Luo, C.F. Giese, and W.R. Gentry, J. Chem. Phys. 104 (1996) 1151.

[127] W. Schöllkopf and J. Peter Toennies, J. Chem. Phys. 104 (1996) 1155.

[128] Y.-H. Uang and W.C. Stwalley, J. Chem. Phys. 76 (1982) 5069; S. Nakaichi-Maeda and T.K. Lim, Phys. Rev. A28 (1983) 692; A.K. Motovilov, S.A. Sofianos, and E.A. Kolganova, Chem. Phys. Lett. 275 (1997) 168.

[129] P.F. Bedaque and H.W. Grießhammer, in preparation.

[130] S.N. Yang and W. Glöckle, Phys. Rev. C33 (1986) 1774.

[131] J.L. Friar, D. Hüber, and U. van Kolck, Phys. Rev. C59 (1999) 53.

[132] J.-I. Fujita and H. Miyazawa, Prog. Theor. Phys. 17 (1957) 360.

[133] D.P. Murphy and S.A. Coon, Few-Body Syst. 18 (1995) 73. 
[134] H.T. Coelho, T.K. Das, and M.R. Robilotta, Phys. Rev. C28 (1983) 1812; M.R. Robilotta and H.T. Coelho, Nucl. Phys. A460 (1986) 645.

[135] J. Carlson, V.R. Pandharipande, and R.B. Wiringa, Nucl. Phys. A401 (1983) 59.

[136] B.S. Pudliner, V.R. Pandharipande, J. Carlson, S.C. Pieper, and R.B. Wiringa, Phys. Rev. C56 (1997) 1720; S.C. Pieper, private communication.

[137] J.A. Eden and M.F. Gari, Phys. Rev. C53 (1996) 1510; J.A. Eden, private communication.

[138] D. Hüber, talk at the IUCF Workshop on Few-Nucleon Physics with Stored, Cooled Beams, IUCF, Bloomington, September 1998.

[139] J. Carlson, talk at the IUCF Workshop on Few-Nucleon Physics with Stored, Cooled Beams, IUCF, Bloomington, September 1998.

[140] H.A. Bethe and C. Longmire, Phys. Rev. 77 (1950) 647.

[141] D.B. Kaplan, M.J. Savage, and M.B. Wise, nucl-th/9804032 v3.

[142] M. Binger, nucl-th/9901012.

[143] M.J. Savage and R.P. Springer, Nucl. Phys. A644 (1998) 235.

[144] M.J. Savage, K.A. Scaldeferri, and M.B. Wise, nucl-th/9811029.

[145] D.B. Kaplan, M.J. Savage, R.P. Springer, and M.B. Wise, nucl-th/9807081.

[146] J.-W. Chen, H.W. Grießhammer, M.J. Savage, and R.P. Springer, Nucl. Phys. A644 (1998) 221.

[147] J.-W. Chen, H.W. Grießhammer, M.J. Savage, and R.P. Springer, Nucl. Phys. A644 (1998) 245.

[148] J.-W. Chen, nucl-th/9810021.

[149] S.R. Beane, V. Bernard, T.-S.H. Lee, and U.-G. Meißner, Phys. Rev. C57 (1998) 424.

[150] A. Misra and D.S. Koltun, nucl-th/9810075.

[151] R.M. Rockmore, Phys. Lett. B356 (1995) 153.

[152] T.-S. Park, D.-P. Min, and M. Rho, Phys. Rev. Lett. 74 (1995) 4153; Nucl. Phys. A596 (1996) 515.

[153] S.R. Beane, D.R. Phillips, M. Malheiro, and U. van Kolck, Washington preprint NT@UW-99-7 in preparation.

[154] S.R. Beane, C.Y. Lee, and U. van Kolck, Phys. Rev. C52 (1995) 2914; S.R. Beane, V. Bernard, T.-S.H. Lee, U.-G. Meißner, and U. van Kolck, Nucl. Phys. A618 (1997) 381.

[155] P. Wilhelm, Phys. Rev. C56 (1997) 1215.

[156] J.H. Koch and R.M. Woloshyn, Phys. Rev. C16 (1997) 1968; J.M. Laget, Phys. Rep. 69 (1981) 1.

[157] J.C. Bergstrom et al., Phys. Rev. C57 (1998) 3203.

[158] MAMI Proposal A1/1-96, Spokesperson: R. Neuhausen.

[159] V. Bernard, N. Kaiser, and U.-G. Meißner, nucl-th/9806013.

[160] U. van Kolck, G.A. Miller, and D.O. Riska, Phys. Lett. B388 (1996) 679.

[161] J.L. Friar, G.L. Payne, V.G.J. Stoks, and J.J. de Swart, Phys. Lett. B311 (1993) 4.

[162] H.O. Meyer et al., Phys. Rev. Lett. 65, (1990) 2846; Nucl. Phys. A539 (1992) 633.

[163] A. Bondar et al., Phys. Lett. B356 (1995) 8.

[164] B.Y. Park, F. Myhrer, J.R. Morones, T. Meissner, and K. Kubodera, Phys. Rev. C53 (1996) 1519; T. Sato, T.-S.H. Lee, F. Myhrer, and K. Kubodera, Phys. Rev. C56 (1997) 1246; C. Hanhart, J. Haidenbauer, M. Hoffmann, U.-G. Meißner, and J. Speth, Phys. 
Lett. B424 (1998) 8; E. Gedalin, A. Moalem, and L. Razdolskaya, hep-ph/9702406; nucl-th/9803029; nucl-th/9812009.

[165] C.A. da Rocha, G.A. Miller, and U. van Kolck, Washington preprint NT@UW-98-10 in preparation.

[166] E. Braaten and A. Nieto, Phys. Rev. B56 (1997) 14745; Phys. Rev. B55 (1997) 8090.

[167] T. Papenbrock and G.F. Bertsch, nucl-th/9811077.

[168] G. Gelmini and B. Ritzi, Phys. Lett. B357 (1995) 431.

[169] J.L. Friar, D.G. Madland, and B.W. Lynn, Phys. Rev. C53 (1996) 3085.

[170] J.J. Rusnak and R.J. Furnstahl, Nucl. Phys. A627 (1997) 495.

[171] R.J. Furnstahl and J.C. Hackworth, Phys. Rev. C56 (1997) 2875.

[172] B.D. Serot and J.D. Walecka, Int. J. Mod. Phys. E6 (1997) 515.

[173] M. Lutz, Nucl. Phys. A642 (1998) 171c. 